\title{
TOP500 Supercomputer Sites
}

16th Edition

\author{
Hans W. Meuer \\ Computing Center \\ University of Mannheim \\ D-68131 Mannheim \\ Germany \\ meuer@rz.uni-mannheim.de \\ Erich Strohmaier \\ Computer Science Department \\ University of Tennessee \\ Knoxville, TN 37996-1301 \\ erich@cs.utk.edu \\ Jack J. Dongarra \\ Computer Science Department \\ University of Tennessee \\ Knoxville, TN 37996-1301 \\ dongarra@cs.utk.edu \\ Horst D. Simon \\ NERSC \\ Lawrence Berkeley National Laboratory \\ Berkeley, CA 94720 \\ simon@nersc.gov
}

November 2, 2000 


\title{
TOP500 Supercomputer Sites
}

\author{
Hans W. Meuer, Erich Strohmaier, Jack J. Dongarra, and Horst \\ D. Simon
}

November 2, 2000

\begin{abstract}
To provide a better basis for statistics on high-performance computers, we list the sites that have the 500 most powerful computer systems installed. The best LiNPACK benchmark performance achieved is used as a performance measure in ranking the computers.
\end{abstract}

\section{Introduction and Objectives}

Statistics on high-performance computers are of major interest to manufacturers, users, and potential users. These people wish to know not only the number of systems installed, but also the location of the various supercomputers within the high-performance computing community and the applications for which a computer system is being used. Such statistics can facilitate the establishment of collaborations, the exchange of data and software, and provide a better understanding of the high-performance computer market.

Statistical lists of supercomputers are not new. Every year since 1986 Hans Meuer [1] has published system counts of the major vector computer manufacturers, based principally on those at the Mannheim Supercomputer Seminar. Statistics based merely on the name of the manufacturer are no longer useful, however. New statistics are required that reflect the diversification of supercomputers, the enormous performance difference between low-end and high-end models, the increasing availability of massively parallel processing (MPP) systems, and the strong increase in computing power of the high-end models of workstation suppliers (SMP).

To provide this new statistical foundation, we have decided in 1993 to assemble and maintain a list of the 500 most powerful computer systems. Our list has been compiled twice a year since June 1993 with the help of high-performance computer experts, computational scientists, manufacturers, and the Internet community in general who responded to a questionnaire we sent out; we thank all the contributors for their cooperation.

In the present list (which we call the Top500), we list computers ranked by their performance on the LINPACK Benchmark. While we make every attempt to verify the results obtained from users and vendors, errors are bound to exist and should be brought to our attention. We intend to continue to update this list half-yearly and, in this way, to keep track with the evolution of computers. Hence, we welcome any comments and information; please send electronic mail 
to top500@rz.uni-mannheim.de. The list is freely available on the WWW at www.top500.org. The interested reader can additionally create sublists out of the ToP500 database and can make statistics on his own by using the WWW interface at

http://www.top500.org . Here you also have access to postscript versions of slides dealing with the interpretation of the present situation as well as with the evolution over time since we started this project.

\section{The LINPACK Benchmark}

As a yardstick of performance we are using the "best" performance as measured by the LinPACK Benchmark [2]. LinPACK was chosen because it is widely used and performance numbers are available for almost all relevant systems.

The LiNPACK Benchmark was introduced by Jack Dongarra. A detailed description as well as a list of performance results on a wide variety of machines is available in postscript form from netlib. To retrieve a copy send electronic mail to netlib@ornl.gov and by typing the message send performance from benchmark or from any machine on the internet type:

rcpanon@netlib2.cs.utk.edu:benchmark/performance performance.

The benchmark used in the LinPACK Benchmark is to solve a dense system of linear equations. For the Top500, we used that version of the benchmark that allows the user to scale the size of the problem and to optimize the software in order to achieve the best performance for a given machine. This performance does not reflect the overall performance of a given system, as no single number ever can. It does, however, reflect the performance of a dedicated system for solving a dense system of linear equations. Since the problem is very regular, the performance achieved is quite high, and the performance numbers give a good correction of peak performance.

By measuring the actual performance for different problem sizes $n$, a user can get not only the maximal achieved performance $R_{\max }$ for the problem size $N_{\max }$ but also the problem size $N_{1 / 2}$ where half of the performance $R_{\max }$ is achieved. These numbers together with the theoretical peak performance $R_{\text {peak }}$ are the numbers given in the TOP500. In an attempt to obtain uniformity across all computers in performance reporting, the algorithm used in solving the system of equations in the benchmark procedure must confirm to the standard operation count for LU factorization with partial pivoting. In particular, the operation count for the algorithm must be $2 / 3 n^{3}+O\left(n^{2}\right)$ floating point operations. This excludes the use of a fast matrix multiply algorithm like "Strassian's Method". This is done to provide a comparable set of performance numbers across all computers. If in the future a more realistic metric finds widespread usage, so that numbers for all systems in question are available, we may convert to that performance measure. 


\section{The TOP500 List}

Table 1 shows the 500 most powerful commercially available computer systems known to us. To keep the list as compact as possible, we show only a part of our information here:

- $N_{\text {world }}$

- Manufacturer

- Computer

- Installation Site

- Location

- Year

- Field of Application

- \# Proc.

- $R_{\text {max }}$

- $R_{\text {peak }}$

- $N_{\text {max }}$

- $N_{1 / 2}$
Position within the Top500 ranking

Manufacturer or vendor

Type indicated by manufacturer or vendor

Customer

Location and country

Year of installation/last major update

Number of processors ${ }^{1}$

Maximal LINPACK performance achieved

Theoretical peak performance

Problemsize for achieving $R_{\max }$

Problemsize for achieving half of $R_{\max }$

If $R_{\max }$ from Table 3 of the LINPACK Report [2] is not available, we use the TPP performance given in Table 1 of the LINPACK Report [2] for solving a system of 1000 equations. To use a consistent yardstick for all systemwe we do not use results achieved by advanced parallel algorithm as defined in [2]. In case of the Cray T90, C90 and J90 systems we had to use older able 3 or Table 1 results. In a few cases we interpolated between two measured system sizes.

For models where we did not receive the requested data, the performance of the next smaller system measured is used.

If there should be any changes in the performances given in Table 1 we will update them.

In addition to cross checking different sources of information, we select randomly a statistical representative sample of the first 500 systems of our database. For these systems we ask the supplier of the information to establish direct contact between the installation site and us to verify the given information. This gives us basic information about the quality of the list in total.

As the TOP500 should provide a basis for statistics on the market of highperformance computers, we limit the number of systems installed at vendor sites. This is done for each vendor separately by limiting the accumulated performance of systems at vendor sites to a maximum of $5 \%$ of the total accumulated installed performance of this vendor. Rounding is done in favor of the vendor in question.

In Table 1, the computers are ordered first by their $R_{\max }$ value. In the case of equal performances ( $R_{\max }$ value) for different computers, we have chosen to order by $R_{\text {peak }}$. For sites that have the same computer, the order is by memory size and then alphabetically. 
ToP500 Supercomputers - Worldwide

\begin{tabular}{|c|c|c|c|c|c|c|}
\hline $\begin{array}{r}\mathbf{N} \\
\text { world }\end{array}$ & $\begin{array}{l}\text { Manufacturer } \\
\text { Computer }\end{array}$ & $\begin{array}{l}\text { Installation Site } \\
\text { Location/Year }\end{array}$ & $\begin{array}{c}\text { Field of } \\
\text { Application }\end{array}$ & $\begin{array}{c}\# \\
\text { Proc. }\end{array}$ & $\begin{array}{r}\mathbf{R}_{\max } \\
R_{\text {peak }} \\
{[\text { Gflop } / \mathrm{s}]}\end{array}$ & $\begin{array}{r}N_{\max } \\
N_{1 / 2}\end{array}$ \\
\hline 1 & $\begin{array}{c}\text { IBM } \\
\text { ASCI White, SP Power3 } 375 \mathrm{MHz}\end{array}$ & $\begin{array}{c}\text { Lawrence Livermore National Laboratory } \\
\text { Livermore USA / } 2000\end{array}$ & $\begin{array}{l}\text { Research } \\
\text { Energy }\end{array}$ & 8192 & $\begin{array}{r}4938 \\
12288\end{array}$ & $\begin{array}{l}430000 \\
430000\end{array}$ \\
\hline 2 & $\begin{array}{c}\text { Intel } \\
\text { ASCI Red }\end{array}$ & $\begin{array}{l}\text { Sandia National Labs } \\
\text { Albuquerque USA /1999 }\end{array}$ & Research & 9632 & $\begin{array}{r}2379 \\
3207\end{array}$ & $\begin{array}{r}362880 \\
75400\end{array}$ \\
\hline 3 & $\begin{array}{c}\text { IBM } \\
\text { ASCI Blue-Pacific SST, IBM SP 604e }\end{array}$ & $\begin{array}{c}\text { Lawrence Livermore National Laboratory } \\
\text { Livermore USA / } 1999\end{array}$ & $\begin{array}{l}\text { Research } \\
\text { Energy }\end{array}$ & 5808 & $\begin{array}{r}2144 \\
3868\end{array}$ & 431344 \\
\hline 4 & $\begin{array}{c}\text { SGI } \\
\text { ASCI Blue Mountain }\end{array}$ & $\begin{array}{c}\text { Los Alamos National Laboratory } \\
\text { Los Alamos USA / } 1998\end{array}$ & Research & 6144 & $\begin{array}{r}1608 \\
3072\end{array}$ & $\begin{array}{l}374400 \\
138000\end{array}$ \\
\hline 5 & $\begin{array}{c}\text { IBM } \\
\text { SP Power3 } 375 \mathrm{MHz}\end{array}$ & $\begin{array}{c}\text { Naval Oceanographic Office (NAVOCEANO) } \\
\text { Bay Saint Louis USA / } 2000\end{array}$ & $\begin{array}{l}\text { Research } \\
\text { Aerospace }\end{array}$ & 1336 & $\begin{array}{r}1417 \\
2004\end{array}$ & $\begin{array}{r}374000 \\
.\end{array}$ \\
\hline 6 & $\begin{array}{c}\text { IBM } \\
\text { SP Power3 } 375 \mathrm{MHz}\end{array}$ & $\begin{array}{l}\text { National Centers for Environmental Prediction } \\
\text { Camp Spring USA } / 2000\end{array}$ & $\begin{array}{l}\text { Research } \\
\text { Weather }\end{array}$ & 1104 & $\begin{array}{r}1179 \\
1656\end{array}$ & \\
\hline 7 & $\begin{array}{c}\text { Hitachi } \\
\text { SR8000-F1/112 }\end{array}$ & $\begin{array}{l}\text { Leibniz Rechenzentrum } \\
\text { Muenchen Germany /2000 }\end{array}$ & Academic & 112 & $\begin{array}{r}1035 \\
1344\end{array}$ & $\begin{array}{r}120000 \\
15160\end{array}$ \\
\hline 8 & $\begin{array}{c}\text { IBM } \\
\text { SP Power3 } 375 \mathrm{MHz} 8 \text { way }\end{array}$ & $\begin{array}{l}\text { UCSD/San Diego Supercomputer Center } \\
\text { San Diego USA / } 2000\end{array}$ & Research & 1152 & $\begin{array}{l}929 \\
1728\end{array}$ & $\begin{array}{r}220000 \\
62000\end{array}$ \\
\hline 9 & $\begin{array}{c}\text { Hitachi } \\
\text { SR8000-F1/100 }\end{array}$ & $\begin{array}{c}\text { High Energy Accelerator Research Organization /KEK } \\
\text { Tsukuba Japan / } 2000\end{array}$ & Research & 100 & $\begin{array}{l}\mathbf{9 1 7} \\
1200\end{array}$ & $\begin{array}{r}115000 \\
15000\end{array}$ \\
\hline 10 & $\begin{array}{l}\text { Cray Inc. } \\
\text { T3E1200 }\end{array}$ & $\begin{array}{l}\text { Government } \\
\text { USA /1998 }\end{array}$ & Classified & 1084 & $\begin{array}{r}892 \\
1300.8\end{array}$ & \\
\hline 11 & $\begin{array}{l}\text { Cray Inc. } \\
\text { T3E1200 }\end{array}$ & $\begin{array}{l}\text { US Army HPC Research Center at NCS } \\
\text { Minneapolis USA } / 2000\end{array}$ & Research & 1084 & $\begin{array}{r}892 \\
1300.8\end{array}$ & \\
\hline 12 & $\begin{array}{c}\text { Fujitsu } \\
\text { VPP5000/100 }\end{array}$ & $\begin{array}{c}\text { ECMWF } \\
\text { Reading UK /2000 }\end{array}$ & $\begin{array}{l}\text { Research } \\
\text { Weather }\end{array}$ & 100 & $\begin{array}{r}886 \\
960\end{array}$ & $\begin{array}{r}195600 \\
18000\end{array}$ \\
\hline 13 & $\begin{array}{c}\text { Hitachi } \\
\text { SR8000/128 }\end{array}$ & $\begin{array}{l}\text { University of Tokyo } \\
\text { Tokyo Japan /1999 }\end{array}$ & Academic & 128 & $\begin{array}{l}873 \\
1024\end{array}$ & $\begin{array}{r}120000 \\
16000 \\
\end{array}$ \\
\hline 14 & $\begin{array}{l}\text { Cray Inc. } \\
\text { T3E900 }\end{array}$ & $\begin{array}{l}\text { Government } \\
\text { USA /1997 }\end{array}$ & Classified & 1324 & $\begin{array}{r}815 \\
1191.6\end{array}$ & $\begin{array}{r}134400 \\
26880\end{array}$ \\
\hline 15 & $\begin{array}{c}\text { IBM } \\
\text { SP Power3 } 375 \mathrm{MHz}\end{array}$ & $\begin{array}{l}\text { Charles Schwab } \\
\text { USA / } 2000\end{array}$ & $\begin{array}{l}\text { Industry } \\
\text { Finance }\end{array}$ & 768 & $\begin{array}{l}795 \\
1152\end{array}$ & \\
\hline 16 & $\begin{array}{c}\text { IBM } \\
\text { SP Power3 } 375 \mathrm{MHz}\end{array}$ & $\begin{array}{l}\text { North Carolina Supercomputing Center (NCSC) } \\
\text { USA } / 2000\end{array}$ & Academic & 720 & $\begin{array}{l}741 \\
1080\end{array}$ & \\
\hline 17 & $\begin{array}{c}\text { IBM } \\
\text { SP Power3 } 375 \mathrm{MHz}\end{array}$ & $\begin{array}{l}\text { Oak Ridge National Laboratory } \\
\text { Oak Ridge USA / } 2000\end{array}$ & Research & 704 & $\begin{array}{l}723 \\
1056\end{array}$ & $\begin{array}{r}187000 \\
37500\end{array}$ \\
\hline 18 & $\begin{array}{c}\text { Hitachi } \\
\text { SR8000-E1/80 }\end{array}$ & $\begin{array}{l}\text { Japan Meteorological Agency } \\
4 \quad \text { Japan } / 2000\end{array}$ & $\begin{array}{l}\text { Research } \\
\text { Weather }\end{array}$ & 80 & $\begin{array}{r}691.3 \\
768\end{array}$ & $\begin{array}{r}120000 \\
9408\end{array}$ \\
\hline 19 & $\begin{array}{c}\text { SGI } \\
\text { ORIGIN } 2000250 \mathrm{MHz}\end{array}$ & $\begin{array}{l}\text { Los Alamos National Laboratory/ACL } \\
\text { Los Alamos USA / } 1999\end{array}$ & Research & 2048 & $\begin{array}{r}690.9 \\
1024\end{array}$ & $\begin{array}{r}229248 \\
80640\end{array}$ \\
\hline 20 & $\begin{array}{c}\text { IBM } \\
\text { SP Power3 } 375 \mathrm{MHz}\end{array}$ & $\begin{array}{c}\text { NCAR (National Center for Atmospheric Research) } \\
\text { Boulder USA / } 2000\end{array}$ & Research & 668 & $\begin{array}{l}688 \\
1002\end{array}$ & \\
\hline
\end{tabular}

Mannheim/Tennessee November 2, 2000 
Top500 Supercomputers - Worldwide

\begin{tabular}{|c|c|c|c|c|c|c|}
\hline $\begin{array}{r}\mathbf{N} \\
\text { world }\end{array}$ & $\begin{array}{l}\text { Manufacturer } \\
\text { Computer }\end{array}$ & $\begin{array}{l}\text { Installation Site } \\
\text { Location/Year }\end{array}$ & $\begin{array}{c}\text { Field of } \\
\text { Application }\end{array}$ & $\begin{array}{c}\# \\
\text { Proc. }\end{array}$ & $\begin{array}{r}\mathbf{R}_{\max } \\
R_{\text {peak }} \\
{[\text { Gflop } / \mathrm{s}]}\end{array}$ & $\begin{array}{r}N_{\max } \\
N_{1 / 2}\end{array}$ \\
\hline 21 & $\begin{array}{l}\text { Cray Inc. } \\
\text { T3E900 }\end{array}$ & $\begin{array}{c}\text { Naval Oceanographic Office (NAVOCEANO) } \\
\text { Bay Saint Louis USA / } 1999\end{array}$ & $\begin{array}{l}\text { Research } \\
\text { Weather }\end{array}$ & 1084 & $\begin{array}{r}\mathbf{6 7 5} \\
975.6\end{array}$ & \\
\hline 22 & $\begin{array}{l}\text { Cray Inc. } \\
\text { T3E1200 }\end{array}$ & $\begin{array}{c}\text { CSAR at the University of Manchester } \\
\text { Manchester UK / } 2000\end{array}$ & Academic & 812 & $\begin{array}{r}671 \\
974.4\end{array}$ & \\
\hline 23 & $\begin{array}{l}\text { Cray Inc. } \\
\text { T3E1200 }\end{array}$ & $\begin{array}{l}\text { Deutscher Wetterdienst } \\
\text { Offenbach Germany /1999 }\end{array}$ & $\begin{array}{l}\text { Research } \\
\text { Weather }\end{array}$ & 812 & $\begin{array}{r}\mathbf{6 7 1} \\
974.4\end{array}$ & \\
\hline 24 & $\begin{array}{c}\text { Hitachi } \\
\text { SR8000-F1/60 }\end{array}$ & $\begin{array}{c}\text { University of Tokyo/Institute for Solid State Physics } \\
\text { Tokyo Japan } / 2000\end{array}$ & Academic & 60 & $\begin{array}{r}577 \\
720\end{array}$ & $\begin{array}{l}89000 \\
10000\end{array}$ \\
\hline 25 & $\begin{array}{c}\text { IBM } \\
\text { SP Power3 } 375 \mathrm{MHz}\end{array}$ & $\begin{array}{c}\text { Wright-Patterson Air Force Base/DoD ASC } \\
\text { USA } / 2000\end{array}$ & $\begin{array}{l}\text { Research } \\
\text { Defense }\end{array}$ & 528 & $\begin{array}{r}\mathbf{5 5 3} \\
792\end{array}$ & \\
\hline 26 & $\begin{array}{l}\text { Cray Inc. } \\
\text { T3E900 }\end{array}$ & $\begin{array}{l}\text { United Kingdom Meteorological Office } \\
\text { Bracknell UK /1997 }\end{array}$ & $\begin{array}{l}\text { Research } \\
\text { Weather }\end{array}$ & 876 & $\begin{array}{r}\mathbf{5 5 2} \\
788.4\end{array}$ & \\
\hline 27 & $\begin{array}{c}\text { IBM } \\
\text { SP Power3 } 375 \mathrm{MHz} 16 \text { way }\end{array}$ & $\begin{array}{c}\text { IBM } \\
\text { Poughkeepsie USA /2000 }\end{array}$ & Vendor & 512 & $\begin{array}{r}546 \\
768\end{array}$ & $\begin{array}{r}148000 \\
33000\end{array}$ \\
\hline 28 & $\begin{array}{c}\text { IBM } \\
\text { SP Power3 } 375 \mathrm{MHz} 16 \text { way }\end{array}$ & $\begin{array}{l}\text { US Army Research Laboratory (ARL) } \\
\text { Aberdeen USA / } 2000\end{array}$ & Research & 512 & $\begin{array}{r}546 \\
768\end{array}$ & $\begin{array}{r}148000 \\
33000\end{array}$ \\
\hline 29 & $\begin{array}{l}\text { Cray Inc. } \\
\text { T3E1200 }\end{array}$ & $\begin{array}{c}\text { United Kingdom Meteorological Office } \\
\text { Bracknell UK /1999 }\end{array}$ & $\begin{array}{l}\text { Research } \\
\text { Weather }\end{array}$ & 636 & $\begin{array}{r}\mathbf{5 2 6} \\
763.2\end{array}$ & \\
\hline 30 & $\begin{array}{l}\text { Cray Inc. } \\
\text { T3E }\end{array}$ & $\begin{array}{l}\text { NASA/Goddard Space Flight Center } \\
\text { Greenbelt USA / } 2000\end{array}$ & $\begin{array}{l}\text { Research } \\
\text { Weather }\end{array}$ & 1356 & $\begin{array}{r}\mathbf{5 2 5} \\
813\end{array}$ & \\
\hline 31 & $\begin{array}{c}\text { Compaq } \\
\text { AlphaServer SC ES40/EV67 }\end{array}$ & $\begin{array}{c}\text { Compaq Computer Corporation } \\
\text { Littleton USA / } 2000\end{array}$ & $\begin{array}{c}\text { Vendor } \\
\text { Benchmarking }\end{array}$ & 512 & $\begin{array}{r}\mathbf{5 0 7 . 6} \\
683\end{array}$ & $\begin{array}{r}200000 \\
30000\end{array}$ \\
\hline 32 & $\begin{array}{c}\text { Compaq } \\
\text { AlphaServer SC ES40/EV67 }\end{array}$ & $\begin{array}{c}\text { Lawrence Livermore National Laboratory } \\
\text { Livermore USA / } 2000\end{array}$ & Research & 512 & $\begin{array}{r}\mathbf{5 0 7 . 6} \\
683\end{array}$ & $\begin{array}{r}200000 \\
30000\end{array}$ \\
\hline 33 & $\begin{array}{c}\text { Fujitsu } \\
\text { VPP5000/56 }\end{array}$ & $\begin{array}{l}\text { Nagoya University } \\
\text { Nagoya Japan /1999 }\end{array}$ & Academic & 56 & $\begin{array}{r}492 \\
537.6\end{array}$ & $\begin{array}{r}228480 \\
12768\end{array}$ \\
\hline 34 & $\begin{array}{c}\text { IBM } \\
\text { SP PC604e } 332 \mathrm{MHz}\end{array}$ & $\begin{array}{l}\text { Charles Schwab } \\
\text { USA / } 1999\end{array}$ & $\begin{array}{l}\text { Industry } \\
\text { Finance }\end{array}$ & 1504 & $\begin{array}{r}490 \\
998\end{array}$ & \\
\hline 35 & $\begin{array}{c}\text { Fujitsu } \\
\text { VPP800/63 }\end{array}$ & $\begin{array}{l}\text { Kyoto University } \\
\text { Kyoto Japan /1999 }\end{array}$ & Academic & 63 & $\begin{array}{r}482 \\
504\end{array}$ & $\begin{array}{r}234360 \\
12852\end{array}$ \\
\hline 36 & $\begin{array}{c}\text { IBM } \\
\text { ASCI Blue-Pacific CTR, IBM SP 604e }\end{array}$ & $\begin{array}{c}\text { Lawrence Livermore National Laboratory } \\
\text { Livermore USA / } 1998\end{array}$ & $\begin{array}{l}\text { Research } \\
\text { Energy }\end{array}$ & 1344 & $\begin{array}{r}468.2 \\
892\end{array}$ & $\begin{array}{r}205000 \\
65000\end{array}$ \\
\hline 37 & $\begin{array}{c}\text { Hitachi } \\
\text { SR8000/64 }\end{array}$ & $\begin{array}{c}\text { Tsukuba Advanced Computing Center - TACC/AIST } \\
\text { Tsukuba Japan /1999 }\end{array}$ & Research & 64 & $\begin{array}{r}449 \\
512\end{array}$ & $\begin{array}{r}92000 \\
9160\end{array}$ \\
\hline 38 & $\begin{array}{l}\text { Cray Inc. } \\
\text { T3E1200 }\end{array}$ & $\begin{array}{c}\text { Cray Inc. } \\
\text { Chippewa Falls USA /1998 }\end{array}$ & Vendor & 540 & $\begin{array}{r}447 \\
648\end{array}$ & $\begin{array}{r}181440 \\
17280\end{array}$ \\
\hline 39 & $\begin{array}{l}\text { Cray Inc. } \\
\text { T3E1200 }\end{array}$ & $\begin{array}{c}\text { ERDC MSRC } \\
\text { Vicksburg USA /1999 }\end{array}$ & $\begin{array}{l}\text { Research } \\
\text { Mechanics }\end{array}$ & 540 & $\begin{array}{r}447 \\
648\end{array}$ & $\begin{array}{r}181440 \\
17280\end{array}$ \\
\hline 40 & $\begin{array}{l}\text { Cray Inc. } \\
\text { T3E1200 }\end{array}$ & $\begin{array}{c}\text { Forschungszentrum Juelich (FZJ) } \\
\text { Juelich Germany /1999 }\end{array}$ & Research & 540 & $\begin{array}{r}447 \\
648\end{array}$ & $\begin{array}{r}181440 \\
17280\end{array}$ \\
\hline
\end{tabular}


ToP500 Supercomputers - Worldwide

\begin{tabular}{|c|c|c|c|c|c|c|}
\hline $\begin{array}{r}\mathbf{N} \\
\text { world }\end{array}$ & $\begin{array}{l}\text { Manufacturer } \\
\text { Computer }\end{array}$ & $\begin{array}{l}\text { Installation Site } \\
\text { Location/Year }\end{array}$ & $\begin{array}{c}\text { Field of } \\
\text { Application }\end{array}$ & $\begin{array}{c}\# \\
\text { Proc. }\end{array}$ & $\begin{array}{r}\mathbf{R}_{\max } \\
R_{\text {peak }} \\
\text { [Gflop/s] }\end{array}$ & $\begin{array}{r}N_{\max } \\
N_{1 / 2}\end{array}$ \\
\hline 41 & $\begin{array}{l}\text { Cray Inc. } \\
\text { T3E1200 }\end{array}$ & $\begin{array}{c}\text { Government } \\
\text { USA / } 1998\end{array}$ & Classified & 540 & $\begin{array}{r}447 \\
648\end{array}$ & $\begin{array}{r}181440 \\
17280\end{array}$ \\
\hline 42 & $\begin{array}{c}\text { Cray Inc. } \\
\text { T3E900 }\end{array}$ & $\begin{array}{c}\text { NERSC/LBNL } \\
\text { Berkeley USA /1997 }\end{array}$ & Research & 692 & $\begin{array}{r}\mathbf{4 4 4} \\
622.8\end{array}$ & \\
\hline 43 & $\begin{array}{c}\text { IBM } \\
\text { SP Power3 } 375 \mathrm{MHz}\end{array}$ & $\begin{array}{c}\text { Financial Institution } \\
\text { Hong Kong / } 2000\end{array}$ & $\begin{array}{l}\text { Industry } \\
\text { Finance }\end{array}$ & 424 & $\begin{array}{r}441 \\
636\end{array}$ & \\
\hline 44 & $\begin{array}{c}\text { Sun } \\
\text { HPC } 4500400 \mathrm{MHz} \text { Cluster }\end{array}$ & $\begin{array}{c}\text { Defense } \\
\text { Stockholm Sweden /1999 }\end{array}$ & Classified & 896 & $\begin{array}{r}\mathbf{4 2 0 . 4 4} \\
716.8\end{array}$ & $\begin{array}{r}144000 \\
43200\end{array}$ \\
\hline 45 & $\begin{array}{c}\text { Sun } \\
\text { HPC } 4500400 \mathrm{MHz} \text { Cluster }\end{array}$ & $\begin{array}{c}\text { Service Provider } \\
\text { USA / } 2000\end{array}$ & $\begin{array}{l}\text { Industry } \\
\text { WWW }\end{array}$ & 896 & $\begin{array}{r}\mathbf{4 2 0 . 4 4} \\
716.8\end{array}$ & $\begin{array}{r}144000 \\
43200\end{array}$ \\
\hline 46 & $\begin{array}{c}\text { Sun } \\
\text { HPC } 4500400 \mathrm{MHz} \text { Cluster }\end{array}$ & $\begin{array}{c}\text { Sun } \\
\text { Burlington USA /2000 }\end{array}$ & Vendor & 896 & $\begin{array}{r}\mathbf{4 2 0 . 4 4} \\
716.8\end{array}$ & $\begin{array}{r}144000 \\
43200\end{array}$ \\
\hline 47 & $\begin{array}{c}\text { Sun } \\
\text { HPC } 4500400 \mathrm{MHz} \text { Cluster }\end{array}$ & $\begin{array}{c}\text { Sun } \\
\text { Sunnyvale USA /2000 }\end{array}$ & Vendor & 896 & $\begin{array}{r}\mathbf{4 2 0 . 4 4} \\
716.8\end{array}$ & $\begin{array}{r}144000 \\
43200\end{array}$ \\
\hline 48 & $\begin{array}{c}\text { IBM } \\
\text { SP Power3 } 375 \mathrm{MHz}\end{array}$ & $\begin{array}{c}\text { Digiton } \\
\text { Finland } / 2000\end{array}$ & Industry & 404 & $\begin{array}{r}420 \\
606\end{array}$ & \\
\hline 49 & $\begin{array}{l}\text { Hitachi/Tsukuba } \\
\text { CP-PACS } / 2048\end{array}$ & $\begin{array}{c}\text { Center for Computational Physics, Univ of Tsukuba } \\
\text { Tsukuba Japan /1996 }\end{array}$ & Academic & 2048 & $\begin{array}{r}368.2 \\
614\end{array}$ & $\begin{array}{r}103680 \\
30720\end{array}$ \\
\hline 50 & $\begin{array}{l}\text { Cray Inc. } \\
\text { T3E }\end{array}$ & $\begin{array}{l}\text { Max-Planck-Gesellschaft MPI/IPP } \\
\text { Garching Germany /1997 }\end{array}$ & Research & 812 & $\begin{array}{r}355 \\
487\end{array}$ & \\
\hline 51 & $\begin{array}{l}\text { Cray Inc. } \\
\text { T3E900 }\end{array}$ & $\begin{array}{l}\text { HWW/Universitaet Stuttgart } \\
\text { Stuttgart Germany /1996 }\end{array}$ & Industry & 540 & $\begin{array}{r}341 \\
486\end{array}$ & \\
\hline 52 & $\begin{array}{l}\text { Cray Inc. } \\
\text { T3E900 }\end{array}$ & $\begin{array}{c}\text { Pittsburgh Supercomputer Center } \\
\text { Pittsburgh USA / } 1998\end{array}$ & Research & 540 & $\begin{array}{r}341 \\
486\end{array}$ & \\
\hline 53 & $\begin{array}{c}\text { IBM } \\
\text { SP Power3 } 222 \mathrm{MHz}\end{array}$ & $\begin{array}{l}\text { European Patent Office } \\
\quad \text { Austria / } 2000\end{array}$ & Government & 562 & $\begin{array}{r}334 \\
499\end{array}$ & \\
\hline 54 & $\begin{array}{l}\text { Cray Inc. } \\
\text { T3E1200 }\end{array}$ & $\begin{array}{l}\text { Government } \\
\text { USA /1999 }\end{array}$ & Classified & 404 & $\begin{array}{r}334 \\
484.8\end{array}$ & \\
\hline 55 & $\begin{array}{c}\text { IBM } \\
\text { SP Power3 } 375 \mathrm{MHz}\end{array}$ & $\begin{array}{c}\text { University of Minnesota/Supercomputing Institute } \\
\text { Minneapolis USA / } 2000\end{array}$ & Academic & 322 & $\begin{array}{r}330 \\
483\end{array}$ & . \\
\hline 56 & $\begin{array}{l}\text { Cray Inc. } \\
\text { T3E1200 }\end{array}$ & $\begin{array}{l}\text { Government } \\
\text { USA / } 2000\end{array}$ & Classified & 396 & $\begin{array}{r}\mathbf{3 2 8} \\
475.2\end{array}$ & . \\
\hline 57 & $\begin{array}{c}\text { IBM } \\
\text { SP Power3 } 375 \mathrm{MHz}\end{array}$ & $\begin{array}{l}\text { Maui High-Performance Computing Center (MHPCC) } \\
\text { USA } / 2000\end{array}$ & Research & 320 & $\begin{array}{r}327 \\
480\end{array}$ & \\
\hline 58 & $\begin{array}{c}\text { IBM } \\
\text { SP Power3 } 375 \mathrm{MHz}\end{array}$ & $\begin{array}{l}\text { BM F LV STAB LRBS } \\
\text { Austria } / 2000\end{array}$ & Government & 318 & $\begin{array}{r}325 \\
477\end{array}$ & \\
\hline 59 & $\begin{array}{c}\text { Fujitsu } \\
\text { VPP700/160E }\end{array}$ & $\begin{array}{c}\text { Institute of Physical and Chemical Res. (RIKEN) } \\
\text { Wako Japan /1999 }\end{array}$ & Research & 160 & $\begin{array}{r}319 \\
384\end{array}$ & $\begin{array}{r}168000 \\
24000\end{array}$ \\
\hline 60 & $\begin{array}{c}\text { IBM } \\
\text { SP Power3 } 200 \mathrm{MHz}\end{array}$ & $\begin{array}{c}\text { NERSC/LBNL } \\
\text { Berkeley USA /1999 }\end{array}$ & Research & 604 & $\begin{array}{r}\mathbf{3 1 0 . 3} \\
483.2\end{array}$ & \\
\hline
\end{tabular}


Top500 Supercomputers - Worldwide

\begin{tabular}{|c|c|c|c|c|c|c|}
\hline $\begin{array}{r}\mathbf{N} \\
\text { world }\end{array}$ & $\begin{array}{l}\text { Manufacturer } \\
\text { Computer }\end{array}$ & $\begin{array}{l}\text { Installation Site } \\
\text { Location/Year }\end{array}$ & $\begin{array}{c}\text { Field of } \\
\text { Application }\end{array}$ & $\begin{array}{c}\# \\
\text { Proc. }\end{array}$ & $\begin{array}{r}\mathbf{R}_{\text {max }} \\
R_{\text {peak }} \\
\text { [Gflop/s] }\end{array}$ & $\begin{array}{r}N_{\max } \\
N_{1 / 2}\end{array}$ \\
\hline 61 & $\begin{array}{c}\text { IBM } \\
\text { SP Power3 } 222 \mathrm{MHz}\end{array}$ & $\begin{array}{c}\text { ERDC MSRC } \\
\text { Vicksburg USA / } 2000\end{array}$ & Research & 512 & $\begin{array}{r}\mathbf{3 0 7 . 6} \\
454.6\end{array}$ & $\begin{array}{r}148000 \\
35000\end{array}$ \\
\hline 62 & $\begin{array}{c}\text { SGI } \\
\text { ORIGIN } 2000400 \mathrm{MHz}\end{array}$ & $\begin{array}{c}\text { NASA/Ames Research Center/NAS } \\
\text { Mountain View USA / } 2000\end{array}$ & $\begin{array}{l}\text { Research } \\
\text { Aerospace }\end{array}$ & 512 & $\begin{array}{r}\mathbf{3 0 0 . 2} \\
409.6\end{array}$ & $\begin{array}{r}130560 \\
21216\end{array}$ \\
\hline 63 & $\begin{array}{c}\text { Fujitsu } \\
\text { VPP5000/32 }\end{array}$ & $\begin{array}{l}\text { Central Research Institute of Electric Power Industry/CRIEPI } \\
\text { Japan } / 2000\end{array}$ & Research & 32 & $\begin{array}{r}\mathbf{2 9 6 . 1} \\
307.2\end{array}$ & $\begin{array}{r}170880 \\
7680\end{array}$ \\
\hline 64 & $\begin{array}{c}\text { IBM } \\
\text { SP Power3 } 375 \mathrm{MHz}\end{array}$ & $\begin{array}{c}\text { US Army Space and Missile Defense Command } \\
\text { Arlington USA / } 2000\end{array}$ & Research & 284 & $\begin{array}{r}288 \\
426\end{array}$ & \\
\hline 65 & $\begin{array}{l}\text { Fujitsu } \\
\text { VPP5000/31 }\end{array}$ & $\begin{array}{l}\text { Meteo-France } \\
\text { Toulouse France /1999 }\end{array}$ & $\begin{array}{l}\text { Research } \\
\text { Weather }\end{array}$ & 31 & $\begin{array}{r}\mathbf{2 8 6} \\
297.6\end{array}$ & . \\
\hline 66 & $\begin{array}{l}\text { Cray Inc. } \\
\text { T3E750 }\end{array}$ & $\begin{array}{l}\text { CSC (Center for Scientific Computing) } \\
\text { Espoo Finland } / 2000\end{array}$ & Academic & 540 & $\begin{array}{r}284 \\
405\end{array}$ & . \\
\hline 67 & $\begin{array}{c}\text { NEC } \\
\text { SX-5/38M3 }\end{array}$ & $\begin{array}{c}\text { CNRS/IDRIS } \\
\text { Orsay France } / 2000\end{array}$ & Academic & 38 & $\begin{array}{r}280 \\
304\end{array}$ & . \\
\hline 68 & $\begin{array}{c}\text { Fujitsu } \\
\text { VPP5000/30 }\end{array}$ & $\begin{array}{c}\text { National Inst. for Molecular Science } \\
\text { Okazaki Japan /2000 }\end{array}$ & Research & 30 & $\begin{array}{r}277 \\
288\end{array}$ & . \\
\hline 69 & $\begin{array}{c}\text { SGI } \\
\text { ORIGIN } 2000195 / 250 \mathrm{MHz}\end{array}$ & $\begin{array}{c}\text { NCSA } \\
\text { Urbana-Champaign USA /1998 }\end{array}$ & Research & 1024 & $\begin{array}{r}\mathbf{2 6 4 . 9} \\
327.68\end{array}$ & . \\
\hline 70 & $\begin{array}{c}\text { Compaq } \\
\text { AlphaServer SC ES40/EV67 }\end{array}$ & $\begin{array}{c}\text { Oak Ridge National Laboratory } \\
\text { Oak Ridge USA / } 2000\end{array}$ & Research & 256 & $\begin{array}{r}263.6 \\
342\end{array}$ & $\begin{array}{r}106000 \\
20000\end{array}$ \\
\hline 71 & $\begin{array}{c}\text { Compaq } \\
\text { AlphaServer SC ES40/EV67 }\end{array}$ & $\begin{array}{c}\text { Pittsburgh Supercomputer Center } \\
\text { Pittsburgh USA / } 2000\end{array}$ & Research & 256 & $\begin{array}{r}263.6 \\
342\end{array}$ & $\begin{array}{r}106000 \\
20000\end{array}$ \\
\hline 72 & $\begin{array}{c}\text { IBM } \\
\text { SP Power3 } 375 \mathrm{MHz}\end{array}$ & $\begin{array}{c}\text { Purdue University } \\
\text { West Lafayette USA /2000 }\end{array}$ & Academic & 256 & $\begin{array}{r}257 \\
384\end{array}$ & $\begin{array}{r}148000 \\
24000\end{array}$ \\
\hline 73 & $\begin{array}{c}\text { Hitachi } \\
\text { SR8000/36 }\end{array}$ & $\begin{array}{c}\text { Meteorological Research Institute } \\
\text { Japan /1999 }\end{array}$ & $\begin{array}{l}\text { Research } \\
\text { Weather }\end{array}$ & 36 & $\begin{array}{r}255 \\
288\end{array}$ & $\begin{array}{r}69000 \\
5968\end{array}$ \\
\hline 74 & $\begin{array}{l}\text { Cray Inc. } \\
\text { T3E900 }\end{array}$ & $\begin{array}{c}\text { ZIB/Konrad Zuse-Zentrum fuer Informationstechnik } \\
\text { Berlin Germany /1999 }\end{array}$ & Academic & 404 & $\begin{array}{r}253 \\
363.6\end{array}$ & . \\
\hline 75 & $\begin{array}{c}\text { IBM } \\
\text { SP PC604e } 332 \mathrm{MHz}\end{array}$ & $\begin{array}{c}\text { Bayer AG } \\
\text { Germany } / 2000\end{array}$ & $\begin{array}{l}\text { Industry } \\
\text { Chemistry }\end{array}$ & 716 & $\begin{array}{r}250 \\
475\end{array}$ & . \\
\hline 76 & $\begin{array}{c}\text { NEC } \\
\text { SX-4/128H4 }\end{array}$ & $\begin{array}{l}\text { Tohoku University } \\
\text { Aramaki Japan /1997 }\end{array}$ & Academic & 128 & $\begin{array}{r}244 \\
256\end{array}$ & . \\
\hline 77 & $\begin{array}{c}\text { NEC } \\
\text { SX-5/32M2 }\end{array}$ & $\begin{array}{c}\text { Bureau of Meteorology / CSIRO HPCCC } \\
\text { Melbourne Australia / } 2000\end{array}$ & $\begin{array}{l}\text { Research } \\
\text { Weather }\end{array}$ & 32 & $\begin{array}{r}243 \\
256\end{array}$ & . \\
\hline 78 & $\begin{array}{c}\text { NEC } \\
\text { SX-5/32M2 }\end{array}$ & $\begin{array}{c}\text { Meteorological Service of Canada (MSC) } \\
\text { Dorval Canada / } 1999\end{array}$ & $\begin{array}{l}\text { Research } \\
\text { Weather }\end{array}$ & 32 & $\begin{array}{r}243 \\
256\end{array}$ & . \\
\hline 79 & $\begin{array}{c}\text { NEC } \\
\mathrm{SX}-5 / 32 \mathrm{H} 2\end{array}$ & $\begin{array}{c}\text { National Research Institute for Metals } \\
\text { Tsukuba Japan } / 2000\end{array}$ & Research & 32 & $\begin{array}{r}243 \\
256\end{array}$ & \\
\hline 80 & $\begin{array}{c}\text { IBM } \\
\text { LosLobos }\end{array}$ & $\begin{array}{c}\text { University of New Mexico } \\
\text { USA / } 2000\end{array}$ & Academic & 512 & $\begin{array}{r}237 \\
375\end{array}$ & $\begin{array}{r}150000 \\
20000\end{array}$ \\
\hline
\end{tabular}

Mannheim/Tennessee November 2, 2000 
TOP500 Supercomputers - Worldwide

\begin{tabular}{|c|c|c|c|c|c|c|}
\hline $\begin{array}{r}\mathbf{N} \\
\text { world }\end{array}$ & $\begin{array}{l}\text { Manufacturer } \\
\text { Computer }\end{array}$ & $\begin{array}{l}\text { Installation Site } \\
\text { Location/Year }\end{array}$ & $\begin{array}{c}\text { Field of } \\
\text { Application }\end{array}$ & $\begin{array}{c}\# \\
\text { Proc. }\end{array}$ & $\begin{array}{r}\mathbf{R}_{\max } \\
R_{\text {peak }} \\
\text { [Gflop/s] }\end{array}$ & $\begin{array}{r}N_{\max } \\
N_{1 / 2}\end{array}$ \\
\hline 81 & $\begin{array}{l}\text { Cray Inc. } \\
\text { T3E1200 }\end{array}$ & $\begin{array}{l}\text { Government } \\
\text { USA /1999 }\end{array}$ & Classified & 284 & $\begin{array}{r}235 \\
340.8\end{array}$ & \\
\hline 82 & $\begin{array}{l}\text { Cray Inc. } \\
\text { T3E }\end{array}$ & $\begin{array}{c}\text { Cray Inc. } \\
\text { Eagan USA /1997 }\end{array}$ & Vendor & 540 & $\begin{array}{r}234 \\
324\end{array}$ & $\begin{array}{l}86400 \\
14400\end{array}$ \\
\hline 83 & $\begin{array}{l}\text { Cray Inc. } \\
\text { T3E }\end{array}$ & $\begin{array}{c}\text { Forschungszentrum Juelich (FZJ) } \\
\text { Juelich Germany /1996 }\end{array}$ & Research & 540 & $\begin{array}{r}\mathbf{2 3 4} \\
324\end{array}$ & $\begin{array}{l}86400 \\
14400\end{array}$ \\
\hline 84 & $\begin{array}{c}\text { Self-made } \\
\text { CPlant Cluster }\end{array}$ & $\begin{array}{c}\text { Sandia National Laboratories } \\
\text { Albuquerque USA /1999 }\end{array}$ & Research & 580 & $\begin{array}{r}232.6 \\
580\end{array}$ & \\
\hline 85 & $\begin{array}{c}\text { Hitachi } \\
\text { SR2201/1024 }\end{array}$ & $\begin{array}{l}\text { University of Tokyo } \\
\text { Tokyo Japan /1996 }\end{array}$ & Academic & 1024 & $\begin{array}{r}232.4 \\
307\end{array}$ & $\begin{array}{r}155520 \\
34560\end{array}$ \\
\hline 86 & $\begin{array}{c}\text { Fujitsu } \\
\text { Numerical Wind Tunnel }\end{array}$ & $\begin{array}{c}\text { NAL } \\
\text { Japan /1996 }\end{array}$ & $\begin{array}{l}\text { Research } \\
\text { Aerospace }\end{array}$ & 167 & $\begin{array}{r}229 \\
281\end{array}$ & $\begin{array}{l}66132 \\
18018\end{array}$ \\
\hline 87 & $\begin{array}{c}\text { Hitachi } \\
\text { SR8000/32 }\end{array}$ & $\begin{array}{l}\text { Hokkaido University } \\
\text { Sapporo Japan /2000 }\end{array}$ & Academic & 32 & $\begin{array}{r}229 \\
256\end{array}$ & $\begin{array}{r}65000 \\
5632\end{array}$ \\
\hline 88 & $\begin{array}{l}\text { Cray Inc. } \\
\text { T3E1200 }\end{array}$ & $\begin{array}{c}\text { CINECA } \\
\text { Bologna Italy } / 1999\end{array}$ & Academic & 268 & $\begin{array}{r}\mathbf{2 2 1 . 7 7} \\
321.6\end{array}$ & \\
\hline 89 & $\begin{array}{l}\text { Cray Inc. } \\
\text { T3E900 }\end{array}$ & $\begin{array}{l}\text { University of Edinburgh } \\
\text { Edinburgh UK / } 1997\end{array}$ & Academic & 348 & $\begin{array}{r}218 \\
313.2\end{array}$ & \\
\hline 90 & $\begin{array}{l}\text { Fujitsu } \\
\text { VPP700/116 }\end{array}$ & $\begin{array}{c}\text { ECMWF } \\
\text { Reading UK /1997 }\end{array}$ & $\begin{array}{l}\text { Research } \\
\text { Weather }\end{array}$ & 116 & $\begin{array}{r}213 \\
255.2\end{array}$ & $\begin{array}{r}111360 \\
18560\end{array}$ \\
\hline 91 & $\begin{array}{c}\text { IBM } \\
\text { SP Power3 } 375 \mathrm{MHz}\end{array}$ & $\begin{array}{c}\text { Bayer AG } \\
\text { Germany } / 2000\end{array}$ & $\begin{array}{l}\text { Industry } \\
\text { Chemistry }\end{array}$ & 210 & $\begin{array}{r}212 \\
315\end{array}$ & \\
\hline 92 & $\begin{array}{c}\text { Compaq } \\
\text { AlphaServer SC ES40/EV67 }\end{array}$ & $\begin{array}{c}\text { Commissariat a l'Energie Atomique (CEA) } \\
\text { Grenoble France / } 1999\end{array}$ & $\begin{array}{l}\text { Research } \\
\text { Energy }\end{array}$ & 232 & $\begin{array}{r}211 \\
309.5\end{array}$ & 120000 \\
\hline 93 & $\begin{array}{c}\text { IBM } \\
\text { SP PC604e } 332 \mathrm{MHz}\end{array}$ & $\begin{array}{c}\text { Sobeys Canada } \\
\text { Nova Scotia Canada /2000 }\end{array}$ & $\begin{array}{l}\text { Industry } \\
\text { Database }\end{array}$ & 512 & $\begin{array}{r}210.2 \\
339.9\end{array}$ & $\begin{array}{r}100000 \\
20872\end{array}$ \\
\hline 94 & $\begin{array}{c}\text { IBM } \\
\text { SP Power3 } 375 \mathrm{MHz}\end{array}$ & $\begin{array}{l}\text { Saudi ARAMCO } \\
\text { Saudi Arabia /2000 }\end{array}$ & $\begin{array}{l}\text { Industry } \\
\text { Geophysics }\end{array}$ & 206 & $\begin{array}{r}208 \\
309\end{array}$ & \\
\hline 95 & $\begin{array}{l}\text { Hewlett-Packard } \\
\text { V2600/HyperPlex }\end{array}$ & $\begin{array}{c}\text { Hewlett-Packard } \\
\text { Richardson USA /2000 }\end{array}$ & $\begin{array}{c}\text { Vendor } \\
\text { Benchmarking }\end{array}$ & 256 & $\begin{array}{r}196 \\
565.24\end{array}$ & \\
\hline 96 & $\begin{array}{c}\text { HPTi } \\
\text { ACL-276 } 667 \mathrm{MHz}\end{array}$ & $\begin{array}{c}\text { Forecast Systems Laboratory/NOAA } \\
\text { Boulder USA / } 1999\end{array}$ & $\begin{array}{l}\text { Research } \\
\text { Weather }\end{array}$ & 276 & $\begin{array}{r}196 \\
368\end{array}$ & 80000 \\
\hline 97 & $\begin{array}{c}\text { SGI } \\
\text { ORIGIN } 2000300 \mathrm{MHz}\end{array}$ & $\begin{array}{c}\text { NASA/Ames Research Center/NAS } \\
\text { Mountain View USA / } 1999\end{array}$ & $\begin{array}{l}\text { Research } \\
\text { Aerospace }\end{array}$ & 512 & $\begin{array}{r}\mathbf{1 9 5 . 6} \\
307.2\end{array}$ & $\begin{array}{r}110592 \\
23040\end{array}$ \\
\hline 98 & $\begin{array}{c}\text { SGI } \\
\text { ORIGIN } 2000300 \mathrm{MHz}\end{array}$ & $\begin{array}{c}\text { Tohoku University, Institute of Fluid Science } \\
\text { Aramaki Japan } / 2000\end{array}$ & Academic & 512 & $\begin{array}{r}\mathbf{1 9 5 . 6} \\
307.2\end{array}$ & $\begin{array}{r}110592 \\
23040\end{array}$ \\
\hline 99 & $\begin{array}{c}\text { IBM } \\
\text { SP PC604e } 332 \mathrm{MHz}\end{array}$ & $\begin{array}{l}\text { Air Force Weather Agency } \\
\text { USA /1999 }\end{array}$ & Research & 440 & $\begin{array}{r}181 \\
292\end{array}$ & \\
\hline 100 & $\begin{array}{c}\text { NEC } \\
\text { SX-5/24M2 }\end{array}$ & $\begin{array}{c}\text { Korea Meteorological Administration (KMA) } \\
\text { Korea } / 2000\end{array}$ & $\begin{array}{l}\text { Research } \\
\text { Weather }\end{array}$ & 24 & $\begin{array}{r}181 \\
192\end{array}$ & \\
\hline
\end{tabular}


Top500 Supercomputers - Worldwide

\begin{tabular}{|c|c|c|c|c|c|c|}
\hline $\begin{array}{r}\mathbf{N} \\
\text { world }\end{array}$ & $\begin{array}{l}\text { Manufacturer } \\
\text { Computer }\end{array}$ & $\begin{array}{l}\text { Installation Site } \\
\text { Location/Year }\end{array}$ & $\begin{array}{c}\text { Field of } \\
\text { Application }\end{array}$ & $\begin{array}{c}\# \\
\text { Proc. }\end{array}$ & $\begin{array}{r}\mathbf{R}_{\max } \\
R_{\text {peak }} \\
\text { [Gflop/s] }\end{array}$ & $\begin{array}{r}N_{\max } \\
N_{1 / 2}\end{array}$ \\
\hline 101 & $\begin{array}{c}\text { IBM } \\
\text { SP P2SC } 120 / 135 \mathrm{MHz}\end{array}$ & $\begin{array}{c}\text { Pacific Northwest National Laboratory } \\
\text { Richland USA / } 1998\end{array}$ & Research & 512 & $\begin{array}{r}180.906 \\
248.32\end{array}$ & 62000 \\
\hline 102 & $\begin{array}{c}\text { IBM } \\
\text { SP Power3 } 375 \mathrm{MHz}\end{array}$ & $\begin{array}{c}\text { Deutsche Telekom AG } \\
\text { Darmstadt Germany /2000 }\end{array}$ & $\begin{array}{l}\text { Industry } \\
\text { Telecomm }\end{array}$ & 168 & $\begin{array}{r}171 \\
252\end{array}$ & \\
\hline 103 & $\begin{array}{c}\text { IBM } \\
\text { SP Power3 } 375 \mathrm{MHz}\end{array}$ & $\begin{array}{l}\text { Florida State University } \\
\text { USA } / 2000\end{array}$ & Academic & 168 & $\begin{array}{r}171 \\
252\end{array}$ & \\
\hline 104 & $\begin{array}{c}\text { IBM } \\
\text { SP Power3 } 375 \mathrm{MHz}\end{array}$ & $\begin{array}{c}\text { National Center for High Performance Computing } \\
\text { HsinChu Taiwan } / 2000\end{array}$ & Academic & 168 & $\begin{array}{r}171 \\
252\end{array}$ & \\
\hline 105 & $\begin{array}{c}\text { IBM } \\
\text { SP Power3 } 375 \mathrm{MHz}\end{array}$ & $\begin{array}{c}\text { PIK } \\
\text { Potsdam Germany } / 2000\end{array}$ & Research & 168 & $\begin{array}{r}171 \\
252\end{array}$ & \\
\hline 106 & $\begin{array}{l}\text { Cray Inc. } \\
\text { T3E900 }\end{array}$ & $\begin{array}{c}\text { Network Computing Services, Inc. } \\
\text { USA / } 1997\end{array}$ & Industry & 268 & $\begin{array}{r}\mathbf{1 6 9} \\
241.2\end{array}$ & \\
\hline 107 & $\begin{array}{l}\text { Cray Inc. } \\
\text { T3E } 900\end{array}$ & $\begin{array}{c}\text { University of Alaska - ARSC } \\
\text { Fairbanks USA /1999 }\end{array}$ & Academic & 268 & $\begin{array}{r}\mathbf{1 6 9} \\
241.2\end{array}$ & \\
\hline 108 & $\begin{array}{c}\text { IBM } \\
\text { SP S80s } 450 \mathrm{MHz}\end{array}$ & $\begin{array}{l}\text { Metro MGI Informatik } \\
\text { Germany } / 2000\end{array}$ & Industry & 510 & $\begin{array}{r}\mathbf{1 6 7 . 8} \\
459\end{array}$ & $\begin{array}{r}113000 \\
31000\end{array}$ \\
\hline 109 & $\begin{array}{c}\text { IBM } \\
\text { SP Power3 } 375 \mathrm{MHz}\end{array}$ & $\begin{array}{l}\text { Air Force Weather Agency } \\
\text { USA } / 2000\end{array}$ & Research & 160 & $\begin{array}{r}164 \\
240\end{array}$ & \\
\hline 110 & $\begin{array}{c}\text { IBM } \\
\text { SP Power3 } 375 \mathrm{MHz}\end{array}$ & $\begin{array}{c}\text { GWDG } \\
\text { Goettingen Germany } / 2000\end{array}$ & Academic & 160 & $\begin{array}{r}164 \\
240\end{array}$ & \\
\hline 111 & $\begin{array}{c}\text { IBM } \\
\text { SP Power3 } 375 \mathrm{MHz}\end{array}$ & $\begin{array}{l}\text { Philip Morris } \\
\text { USA / } 2000\end{array}$ & Industry & 160 & $\begin{array}{r}164 \\
240\end{array}$ & \\
\hline 112 & $\begin{array}{c}\text { IBM } \\
\text { SP PC604e } 332 \mathrm{MHz}\end{array}$ & $\begin{array}{c}\text { Bank Administration Institute (BAI) } \\
\text { USA / } 2000\end{array}$ & $\begin{array}{l}\text { Industry } \\
\text { Finance }\end{array}$ & 396 & $\begin{array}{r}163 \\
262\end{array}$ & \\
\hline 113 & $\begin{array}{c}\text { IBM } \\
\text { SP PC604e } 332 \mathrm{MHz}\end{array}$ & $\begin{array}{c}\text { BCDI } \\
\text { USA } / 2000\end{array}$ & Industry & 392 & $\begin{array}{r}162 \\
260\end{array}$ & \\
\hline 114 & $\begin{array}{c}\text { IBM } \\
\text { SP PC604e } 332 \mathrm{MHz}\end{array}$ & $\begin{array}{l}\text { Metallurgical Industry Co. } \\
\text { USA } / 2000\end{array}$ & Industry & 392 & $\begin{array}{r}162 \\
260\end{array}$ & \\
\hline 115 & $\begin{array}{c}\text { IBM } \\
\text { SP Power3 } 375 \mathrm{MHz}\end{array}$ & $\begin{array}{l}\text { Support Net Inc } \\
\text { USA / } 2000\end{array}$ & Industry & 156 & $\begin{array}{r}160 \\
234\end{array}$ & \\
\hline 116 & $\begin{array}{c}\text { IBM } \\
\text { SP Power3 } 375 \mathrm{MHz} 16 \text { way }\end{array}$ & $\begin{array}{l}\text { Seoul National University } \\
\text { Seoul Korea / } 2000\end{array}$ & Academic & 144 & $\begin{array}{r}156 \\
216\end{array}$ & \\
\hline 117 & $\begin{array}{c}\text { IBM } \\
\text { SP Power3 } 375 \mathrm{MHz}\end{array}$ & $\begin{array}{c}\text { Bayer AG } \\
\text { Austria } / 2000\end{array}$ & $\begin{array}{l}\text { Industry } \\
\text { Chemistry }\end{array}$ & 150 & $\begin{array}{r}154 \\
225\end{array}$ & \\
\hline 118 & $\begin{array}{c}\text { IBM } \\
\text { SP Power3 } 375 \mathrm{MHz}\end{array}$ & $\begin{array}{l}\text { Saudi ARAMCO } \\
\text { Saudi Arabia } / 2000\end{array}$ & $\begin{array}{l}\text { Industry } \\
\text { Geophysics }\end{array}$ & 148 & $\begin{array}{r}152 \\
222\end{array}$ & \\
\hline 119 & $\begin{array}{c}\text { SGI } \\
\text { ORIGIN } 2000\end{array}$ & $\begin{array}{c}\text { Wright-Patterson Air Force Base/DoD ASC } \\
\text { USA / } 1999\end{array}$ & Research & 512 & $\begin{array}{r}152 \\
199.68\end{array}$ & \\
\hline 120 & $\begin{array}{c}\text { IBM } \\
\text { SP Power3 } 200 \mathrm{MHz}\end{array}$ & $\begin{array}{l}\text { State Farm } \\
\text { USA /1999 }\end{array}$ & $\begin{array}{l}\text { Industry } \\
\text { Database }\end{array}$ & 260 & $\begin{array}{r}\mathbf{1 5 1 . 5} \\
208.1\end{array}$ & \\
\hline
\end{tabular}


TOP500 Supercomputers - Worldwide

\begin{tabular}{|c|c|c|c|c|c|c|}
\hline $\begin{array}{r}\mathbf{N} \\
\text { world }\end{array}$ & $\begin{array}{l}\text { Manufacturer } \\
\text { Computer }\end{array}$ & $\begin{array}{l}\text { Installation Site } \\
\text { Location/Year }\end{array}$ & $\begin{array}{c}\text { Field of } \\
\text { Application }\end{array}$ & $\begin{array}{c}\# \\
\text { Proc. }\end{array}$ & $\begin{array}{r}\mathbf{R}_{\max } \\
R_{\text {peak }} \\
\text { [Gflop/s] }\end{array}$ & $\begin{array}{r}N_{\max } \\
N_{1 / 2}\end{array}$ \\
\hline 121 & $\begin{array}{c}\text { IBM } \\
\text { SP PC604e } 332 \mathrm{MHz}\end{array}$ & $\begin{array}{c}\text { BASF } \\
\text { Ludwigshafen Germany /2000 }\end{array}$ & $\begin{array}{l}\text { Industry } \\
\text { Chemistry }\end{array}$ & 364 & $\begin{array}{r}151 \\
241\end{array}$ & \\
\hline 122 & $\begin{array}{c}\text { IBM } \\
\text { SP Power3 } 200 \mathrm{MHz}\end{array}$ & $\begin{array}{c}\text { Tsukuba Advanced Computing Center - TACC/AIST } \\
\text { Tsukuba Japan /1999 }\end{array}$ & Research & 256 & $\begin{array}{r}149.3 \\
205\end{array}$ & $\begin{array}{r}100000 \\
18500\end{array}$ \\
\hline 123 & $\begin{array}{c}\text { IBM } \\
\text { SP Power3 } 375 \mathrm{MHz}\end{array}$ & $\begin{array}{l}\text { Prudential Insurance } \\
\text { USA / } 2000\end{array}$ & $\begin{array}{l}\text { Industry } \\
\text { Finance }\end{array}$ & 144 & $\begin{array}{r}148 \\
216\end{array}$ & \\
\hline 124 & $\begin{array}{c}\text { IBM } \\
\text { SP Power3 } 375 \mathrm{MHz}\end{array}$ & $\begin{array}{l}\text { Manufacturer } \\
\text { Japan /2000 }\end{array}$ & Industry & 142 & $\begin{array}{r}146 \\
213\end{array}$ & \\
\hline 125 & $\begin{array}{c}\text { Hitachi } \\
\text { SR } 8000 / 20\end{array}$ & $\begin{array}{c}\text { Institute of Statistical Mathematics } \\
\text { Tokyo Japan /1999 }\end{array}$ & Research & 20 & $\begin{array}{r}144 \\
160\end{array}$ & $\begin{array}{r}48000 \\
4000\end{array}$ \\
\hline 126 & $\begin{array}{c}\text { Self-made } \\
\text { CLIC PIII } 800 \mathrm{MHz}\end{array}$ & $\begin{array}{l}\text { Technische Universitaet Chemnitz } \\
\text { Chemnitz Germany / } 2000\end{array}$ & Academic & 528 & $\begin{array}{r}\mathbf{1 4 3 . 3} \\
422\end{array}$ & 150000 \\
\hline 127 & $\begin{array}{c}\text { IBM } \\
\text { SP S80s } 450 \mathrm{MHz}\end{array}$ & $\begin{array}{c}\text { Bayer AG } \\
\text { Germany } / 2000\end{array}$ & $\begin{array}{l}\text { Industry } \\
\text { Chemistry }\end{array}$ & 294 & $\begin{array}{r}139 \\
264\end{array}$ & \\
\hline 128 & $\begin{array}{c}\text { Fujitsu } \\
\text { VPP5000/15 }\end{array}$ & $\begin{array}{c}\text { Commissariat a l'Energie Atomique (CEA) } \\
\text { Grenoble France / } 1999\end{array}$ & $\begin{array}{l}\text { Research } \\
\text { Energy }\end{array}$ & 15 & $\begin{array}{r}139 \\
144\end{array}$ & \\
\hline 129 & $\begin{array}{c}\text { Fujitsu } \\
\text { VPP5000/15 }\end{array}$ & $\begin{array}{c}\text { Taiwan Central Weather Bureau } \\
\text { Taipei Taiwan /1999 }\end{array}$ & $\begin{array}{l}\text { Research } \\
\text { Weather }\end{array}$ & 15 & $\begin{array}{r}139 \\
144\end{array}$ & \\
\hline 130 & $\begin{array}{c}\text { IBM } \\
\text { SP PC604e } 332 \mathrm{MHz}\end{array}$ & $\begin{array}{c}\text { DeTeCSM } \\
\text { Germany } / 2000\end{array}$ & $\begin{array}{l}\text { Industry } \\
\text { Telecomm }\end{array}$ & 452 & $\begin{array}{r}138 \\
300\end{array}$ & \\
\hline 131 & $\begin{array}{c}\text { IBM } \\
\text { SP PC604e } 332 \mathrm{MHz}\end{array}$ & $\begin{array}{c}\text { DeTeCSM } \\
\text { Bielefeld Germany } / 2000\end{array}$ & $\begin{array}{l}\text { Industry } \\
\text { Telecomm }\end{array}$ & 452 & $\begin{array}{r}138 \\
300\end{array}$ & \\
\hline 132 & $\begin{array}{c}\text { IBM } \\
\text { SP Power3 } 222 \mathrm{MHz}\end{array}$ & $\begin{array}{c}\text { Centre Informatique National (CINES) } \\
\text { Montpellier France } / 2000\end{array}$ & Academic & 224 & $\begin{array}{r}138 \\
198\end{array}$ & \\
\hline 133 & $\begin{array}{c}\text { Sun } \\
\text { HPC } 10000400 \mathrm{MHz} \text { Cluster }\end{array}$ & $\begin{array}{c}\text { Australian Partnership for Advanced Computing (APAC) } \\
\text { Canberra Australia /2000 }\end{array}$ & Academic & 256 & $\begin{array}{r}\mathbf{1 3 7 . 1} \\
204.8\end{array}$ & \\
\hline 134 & $\begin{array}{c}\text { Sun } \\
\text { HPC } 10000400 \mathrm{MHz} \text { Cluster }\end{array}$ & $\begin{array}{l}\text { Clearstream Services } \\
\text { Grande Duchesse Luxembourg /2000 }\end{array}$ & $\begin{array}{l}\text { Industry } \\
\text { Finance }\end{array}$ & 256 & $\begin{array}{r}\mathbf{1 3 7 . 1} \\
204.8\end{array}$ & \\
\hline 135 & $\begin{array}{c}\text { Sun } \\
\text { HPC } 10000400 \mathrm{MHz} \text { Cluster }\end{array}$ & $\begin{array}{c}\text { Motorola } \\
\text { Scottsdale USA /2000 }\end{array}$ & $\begin{array}{l}\text { Industry } \\
\text { Electronics }\end{array}$ & 256 & $\begin{array}{r}\mathbf{1 3 7 . 1} \\
204.8\end{array}$ & \\
\hline 136 & $\begin{array}{c}\text { Sun } \\
\text { HPC } 10000400 \mathrm{MHz} \text { Cluster }\end{array}$ & $\begin{array}{c}\text { New York City - Human Resources } \\
\text { USA /1999 }\end{array}$ & Government & 256 & $\begin{array}{r}\mathbf{1 3 7 . 1} \\
204.8\end{array}$ & \\
\hline 137 & $\begin{array}{c}\text { Sun } \\
\text { HPC } 10000400 \mathrm{MHz} \text { Cluster }\end{array}$ & $\begin{array}{c}\text { Sun } \\
\text { Broomfield USA /2000 }\end{array}$ & $\begin{array}{l}\text { Industry } \\
\text { WWW }\end{array}$ & 256 & $\begin{array}{r}\mathbf{1 3 7 . 1} \\
204.8\end{array}$ & \\
\hline 138 & $\begin{array}{c}\text { Sun } \\
\text { HPC } 10000400 \mathrm{MHz} \text { Cluster }\end{array}$ & $\begin{array}{l}\text { US Army Research Laboratory (ARL) } \\
\text { Aberdeen USA / } 1999\end{array}$ & Research & 256 & $\begin{array}{r}\mathbf{1 3 7 . 1} \\
204.8\end{array}$ & \\
\hline 139 & $\begin{array}{c}\text { Sun } \\
\text { HPC } 10000400 \mathrm{MHz} \text { Cluster }\end{array}$ & $\begin{array}{c}\text { Bank } \\
\text { Westboro USA /2000 }\end{array}$ & $\begin{array}{l}\text { Industry } \\
\text { Finance }\end{array}$ & 256 & $\begin{array}{r}\mathbf{1 3 7 . 1} \\
204.8\end{array}$ & \\
\hline 140 & $\begin{array}{c}\text { Sun } \\
\text { HPC } 10000400 \mathrm{MHz} \text { Cluster }\end{array}$ & $\begin{array}{c}\text { E-commerce } \\
\text { Santa Clara USA /2000 }\end{array}$ & $\begin{array}{l}\text { Industry } \\
\text { WWW }\end{array}$ & 256 & $\begin{array}{r}\mathbf{1 3 7 . 1} \\
204.8\end{array}$ & \\
\hline
\end{tabular}

Mannheim/Tennessee November 2, 2000 
Top500 Supercomputers - Worldwide

\begin{tabular}{|c|c|c|c|c|c|c|}
\hline $\begin{array}{r}\mathbf{N} \\
\text { world }\end{array}$ & $\begin{array}{l}\text { Manufacturer } \\
\text { Computer }\end{array}$ & $\begin{array}{l}\text { Installation Site } \\
\text { Location/Year }\end{array}$ & $\begin{array}{c}\text { Field of } \\
\text { Application }\end{array}$ & $\begin{array}{c}\# \\
\text { Proc. }\end{array}$ & $\begin{array}{r}\mathbf{R}_{\text {max }} \\
R_{\text {peak }} \\
\text { [Gflop/s] }\end{array}$ & $\begin{array}{r}N_{\max } \\
N_{1 / 2}\end{array}$ \\
\hline 141 & $\begin{array}{c}\text { Sun } \\
\text { HPC } 10000400 \mathrm{MHz} \text { Cluster }\end{array}$ & $\begin{array}{l}\text { Ford Motor Company } \\
\text { Detroit USA / } 2000\end{array}$ & $\begin{array}{l}\text { Industry } \\
\text { Automotive }\end{array}$ & 256 & $\begin{array}{r}\mathbf{1 3 7 . 1} \\
204.8\end{array}$ & \\
\hline 142 & $\begin{array}{c}\text { Sun } \\
\text { HPC } 10000400 \mathrm{MHz} \text { Cluster }\end{array}$ & $\begin{array}{l}\text { GTE Communications } \\
\text { Sacramento USA / } 2000\end{array}$ & $\begin{array}{l}\text { Industry } \\
\text { Telecomm }\end{array}$ & 256 & $\begin{array}{r}\mathbf{1 3 7 . 1} \\
204.8\end{array}$ & \\
\hline 143 & $\begin{array}{c}\text { Sun } \\
\text { HPC } 10000400 \mathrm{MHz} \text { Cluster }\end{array}$ & $\begin{array}{c}\text { GTE Communications } \\
\text { Temple Terrace USA / } 2000\end{array}$ & $\begin{array}{l}\text { Industry } \\
\text { Telecomm }\end{array}$ & 256 & $\begin{array}{r}\mathbf{1 3 7 . 1} \\
204.8\end{array}$ & \\
\hline 144 & $\begin{array}{c}\text { IBM } \\
\text { SP Power3 } 375 \mathrm{MHz}\end{array}$ & $\begin{array}{l}\text { Kaiser Foundation } \\
\text { USA } / 2000\end{array}$ & Industry & 132 & $\begin{array}{r}\mathbf{1 3 6} \\
198\end{array}$ & \\
\hline 145 & $\begin{array}{c}\text { IBM } \\
\text { SP PC604e } 332 \mathrm{MHz}\end{array}$ & $\begin{array}{l}\text { IBM - Thomas Watson Research Center } \\
\text { Yorktown Heights USA / } 2000\end{array}$ & Research & 320 & $\begin{array}{r}133 \\
212\end{array}$ & \\
\hline 146 & $\begin{array}{c}\text { IBM } \\
\text { SP PC604e } 332 \mathrm{MHz}\end{array}$ & $\begin{array}{c}\text { IBM Credit Corporation } \\
\text { USA } / 2000\end{array}$ & Industry & 320 & $\begin{array}{r}133 \\
212\end{array}$ & \\
\hline 147 & $\begin{array}{c}\text { IBM } \\
\text { SP Power3 } 375 \mathrm{MHz}\end{array}$ & $\begin{array}{c}\text { CSC (Centre for Sientific Computing) } \\
\text { Espoo Finland } / 2000\end{array}$ & Academic & 128 & $\begin{array}{r}132 \\
192\end{array}$ & $\begin{array}{r}107000 \\
15400\end{array}$ \\
\hline 148 & $\begin{array}{c}\text { IBM } \\
\text { SP Power3 } 375 \mathrm{MHz}\end{array}$ & $\begin{array}{l}\text { Princeton University } \\
\text { Princeton USA / } 2000\end{array}$ & Academic & 128 & $\begin{array}{r}132 \\
192\end{array}$ & $\begin{array}{r}107000 \\
15400\end{array}$ \\
\hline 149 & $\begin{array}{c}\text { IBM } \\
\text { SP Power3 } 375 \mathrm{MHz}\end{array}$ & $\begin{array}{l}\text { Universitaet/Forschungszentrum Karlsruhe } \\
\text { Karlsruhe Germany / } 2000\end{array}$ & Academic & 128 & $\begin{array}{r}132 \\
192\end{array}$ & $\begin{array}{r}107000 \\
15400\end{array}$ \\
\hline 150 & $\begin{array}{c}\text { IBM } \\
\text { SP Power3 } 375 \mathrm{MHz}\end{array}$ & $\begin{array}{l}\text { Telecom Denmark (Danadata) } \\
\text { Denmark / } 2000\end{array}$ & $\begin{array}{l}\text { Industry } \\
\text { Telecomm }\end{array}$ & 126 & $\begin{array}{r}130 \\
189\end{array}$ & \\
\hline 151 & $\begin{array}{c}\text { IBM } \\
\text { SP Power3 } 375 \mathrm{MHz}\end{array}$ & $\begin{array}{l}\text { Paine Webber } \\
\text { USA / } 2000\end{array}$ & Industry & 124 & $\begin{array}{r}128 \\
186\end{array}$ & \\
\hline 152 & $\begin{array}{c}\text { IBM } \\
\text { SP PC604e } 332 \mathrm{MHz}\end{array}$ & $\begin{array}{l}\text { British Airways } \\
\text { UK /1999 }\end{array}$ & $\begin{array}{l}\text { Industry } \\
\text { Transportation }\end{array}$ & 302 & $\begin{array}{r}126 \\
200\end{array}$ & \\
\hline 153 & $\begin{array}{c}\text { IBM } \\
\text { SP Power3 } 200 \mathrm{MHz}\end{array}$ & $\begin{array}{c}\text { IBM - Thomas Watson Research Center } \\
\text { Yorktown Heights USA / } 2000\end{array}$ & Research & 212 & $\begin{array}{r}\mathbf{1 2 4 . 4} \\
169.5\end{array}$ & \\
\hline 154 & $\begin{array}{c}\text { IBM } \\
\text { SP PC604e } 332 \mathrm{MHz}\end{array}$ & $\begin{array}{l}\text { Philips Lighting } \\
\text { Netherlands /2000 }\end{array}$ & $\begin{array}{l}\text { Industry } \\
\text { Electronics }\end{array}$ & 298 & $\begin{array}{r}124 \\
197\end{array}$ & \\
\hline 155 & $\begin{array}{c}\text { IBM } \\
\text { SP Power3 } 375 \mathrm{MHz}\end{array}$ & $\begin{array}{c}\text { Geco-Prakla } \\
\text { Houston USA / } 2000\end{array}$ & $\begin{array}{l}\text { Industry } \\
\text { Geophysics }\end{array}$ & 120 & $\begin{array}{r}124 \\
180\end{array}$ & \\
\hline 156 & $\begin{array}{c}\text { IBM } \\
\text { SP Power3 } 375 \mathrm{MHz}\end{array}$ & $\begin{array}{l}\text { Western Geophysical } \\
\text { London UK / } 2000\end{array}$ & $\begin{array}{l}\text { Industry } \\
\text { Geophysics }\end{array}$ & 120 & $\begin{array}{r}124 \\
180\end{array}$ & \\
\hline 157 & $\begin{array}{c}\text { NEC } \\
\text { SX-5/16A }\end{array}$ & $\begin{array}{l}\text { Frontier Research System for Global Change } \\
\qquad \text { Japan /1999 }\end{array}$ & Research & 16 & $\begin{array}{r}123 \\
128\end{array}$ & $\begin{array}{r}99840 \\
1340\end{array}$ \\
\hline 158 & $\begin{array}{c}\text { NEC } \\
\text { SX-5/16A }\end{array}$ & $\begin{array}{l}\text { NEC Fuchu Plant } \\
\text { Tokyo Japan /1999 }\end{array}$ & $\begin{array}{c}\text { Vendor } \\
\text { Benchmarking }\end{array}$ & 16 & $\begin{array}{r}\mathbf{1 2 3} \\
128\end{array}$ & $\begin{array}{r}99840 \\
1340\end{array}$ \\
\hline 159 & $\begin{array}{c}\text { NEC } \\
\text { SX-5/16A }\end{array}$ & $\begin{array}{c}\text { ONERA } \\
\text { France } / 1999\end{array}$ & $\begin{array}{l}\text { Research } \\
\text { Aerospace }\end{array}$ & 16 & $\begin{array}{r}\mathbf{1 2 3} \\
128\end{array}$ & $\begin{array}{r}99840 \\
1340\end{array}$ \\
\hline 160 & $\begin{array}{c}\text { NEC } \\
\text { SX-5/16A }\end{array}$ & $\begin{array}{c}\text { Tohoku University, Institute of Fluid Science } \\
\text { Aramaki Japan /1999 }\end{array}$ & Academic & 16 & $\begin{array}{r}\mathbf{1 2 3} \\
128\end{array}$ & $\begin{array}{r}99840 \\
1340\end{array}$ \\
\hline
\end{tabular}

Mannheim/Tennessee November 2, 2000 
Top500 Supercomputers - Worldwide

\begin{tabular}{|c|c|c|c|c|c|c|}
\hline $\begin{array}{r}\mathbf{N} \\
\text { world }\end{array}$ & $\begin{array}{l}\text { Manufacturer } \\
\text { Computer }\end{array}$ & $\begin{array}{l}\text { Installation Site } \\
\text { Location/Year }\end{array}$ & $\begin{array}{c}\text { Field of } \\
\text { Application }\end{array}$ & $\begin{array}{c}\# \\
\text { Proc. }\end{array}$ & $\begin{array}{r}\mathbf{R}_{\text {max }} \\
R_{\text {peak }} \\
\text { [Gflop/s] }\end{array}$ & $\begin{array}{r}N_{\max } \\
N_{1 / 2}\end{array}$ \\
\hline 161 & $\begin{array}{c}\text { NEC } \\
\text { SX-5/16A }\end{array}$ & $\begin{array}{c}\text { Tokyo Institute of Technology } \\
\text { Tokyo Japan /1999 }\end{array}$ & Academic & 16 & $\begin{array}{r}123 \\
128\end{array}$ & $\begin{array}{r}99840 \\
1340\end{array}$ \\
\hline 162 & $\begin{array}{c}\text { IBM } \\
\text { SP PC604e } 332 \mathrm{MHz}\end{array}$ & $\begin{array}{c}\text { Banque National Paris } \\
\text { France } / 2000\end{array}$ & $\begin{array}{l}\text { Industry } \\
\text { Finance }\end{array}$ & 292 & $\begin{array}{r}122 \\
193\end{array}$ & \\
\hline 163 & $\begin{array}{c}\text { NEC } \\
\text { SX-4/64M2 }\end{array}$ & $\begin{array}{c}\text { Meteorological Service of Canada (MSC) } \\
\text { Dorval Canada /1999 }\end{array}$ & $\begin{array}{l}\text { Research } \\
\text { Weather }\end{array}$ & 64 & $\begin{array}{r}122 \\
128\end{array}$ & $\begin{array}{r}30080 \\
4352\end{array}$ \\
\hline 164 & $\begin{array}{c}\text { NEC } \\
\text { SX-4/64M2 }\end{array}$ & $\begin{array}{l}\text { National Institute of Fusion Science (NIFS) } \\
\text { Japan /1997 }\end{array}$ & Research & 64 & $\begin{array}{r}122 \\
128\end{array}$ & $\begin{array}{r}30080 \\
4352\end{array}$ \\
\hline 165 & $\begin{array}{c}\text { NEC } \\
\text { SX-4/64M2 }\end{array}$ & $\begin{array}{l}\text { Osaka University } \\
\text { Osaka Japan /1997 }\end{array}$ & Academic & 64 & $\begin{array}{r}122 \\
128\end{array}$ & $\begin{array}{r}30080 \\
4352\end{array}$ \\
\hline 166 & $\begin{array}{c}\text { Sun } \\
\text { HPC } 420400 \mathrm{MHz} \text { Cluster }\end{array}$ & $\begin{array}{c}\text { Financial Services } \\
\text { New York USA / } 2000\end{array}$ & $\begin{array}{l}\text { Industry } \\
\text { Finance }\end{array}$ & 180 & $\begin{array}{r}121.9 \\
144\end{array}$ & \\
\hline 167 & $\begin{array}{c}\text { NEC } \\
\text { SX-5/32Me2 }\end{array}$ & $\begin{array}{c}\text { HWW/Universitaet Stuttgart } \\
\text { Stuttgart Germany /2000 }\end{array}$ & Industry & 32 & $\begin{array}{r}121 \\
128\end{array}$ & \\
\hline 168 & $\begin{array}{c}\text { IBM } \\
\text { SP PC604e } 332 \mathrm{MHz}\end{array}$ & $\begin{array}{c}\text { Alcatel } \\
\text { France } / 2000\end{array}$ & $\begin{array}{l}\text { Industry } \\
\text { Telecomm }\end{array}$ & 288 & $\begin{array}{r}120 \\
191\end{array}$ & . \\
\hline 169 & $\begin{array}{c}\text { Sun } \\
\text { HPC } 10000400 \mathrm{MHz} \text { Cluster }\end{array}$ & $\begin{array}{c}\text { Airline } \\
\text { London UK /2000 }\end{array}$ & $\begin{array}{c}\text { Industry } \\
\text { Transportation }\end{array}$ & 192 & $\begin{array}{r}\mathbf{1 1 8 . 1 6} \\
153.6\end{array}$ & . \\
\hline 170 & $\begin{array}{c}\text { Sun } \\
\text { HPC } 10000400 \mathrm{MHz} \text { Cluster }\end{array}$ & $\begin{array}{c}\text { Bank } \\
\text { Milano Italy } / 2000\end{array}$ & $\begin{array}{l}\text { Industry } \\
\text { Finance }\end{array}$ & 192 & $\begin{array}{r}\mathbf{1 1 8 . 1 6} \\
153.6\end{array}$ & \\
\hline 171 & $\begin{array}{c}\text { Sun } \\
\text { HPC } 10000400 \mathrm{MHz} \text { Cluster }\end{array}$ & $\begin{array}{c}\text { Bank } \\
\text { Munich Germany /2000 }\end{array}$ & $\begin{array}{l}\text { Industry } \\
\text { Finance }\end{array}$ & 192 & $\begin{array}{r}\mathbf{1 1 8 . 1 6} \\
153.6\end{array}$ & \\
\hline 172 & $\begin{array}{c}\text { Sun } \\
\text { HPC } 10000400 \mathrm{MHz} \text { Cluster }\end{array}$ & $\begin{array}{c}\text { BellSouth } \\
\text { Tucker USA /2000 }\end{array}$ & $\begin{array}{l}\text { Industry } \\
\text { Telecomm }\end{array}$ & 192 & $\begin{array}{r}\mathbf{1 1 8 . 1 6} \\
153.6\end{array}$ & . \\
\hline 173 & $\begin{array}{c}\text { Sun } \\
\text { HPC } 10000400 \mathrm{MHz} \text { Cluster }\end{array}$ & $\begin{array}{c}\text { Chase GlobalNet } \\
\text { USA / } 2000\end{array}$ & $\begin{array}{l}\text { Industry } \\
\text { Finance }\end{array}$ & 192 & $\begin{array}{r}\mathbf{1 1 8 . 1 6} \\
153.6\end{array}$ & \\
\hline 174 & $\begin{array}{c}\text { Sun } \\
\text { HPC } 10000400 \mathrm{MHz} \text { Cluster }\end{array}$ & $\begin{array}{l}\text { Computer Manufacturer } \\
\text { Lakewood USA / } 2000\end{array}$ & $\begin{array}{c}\text { Industry } \\
\text { Manufacturing }\end{array}$ & 192 & $\begin{array}{r}\mathbf{1 1 8 . 1 6} \\
153.6\end{array}$ & \\
\hline 175 & $\begin{array}{c}\text { Sun } \\
\text { HPC } 10000400 \mathrm{MHz} \text { Cluster }\end{array}$ & $\begin{array}{l}\text { Financial Services } \\
\text { London UK / } 2000\end{array}$ & $\begin{array}{l}\text { Industry } \\
\text { Finance }\end{array}$ & 192 & $\begin{array}{r}\mathbf{1 1 8 . 1 6} \\
153.6\end{array}$ & \\
\hline 176 & $\begin{array}{c}\text { Sun } \\
\text { HPC } 10000400 \mathrm{MHz} \text { Cluster }\end{array}$ & $\begin{array}{c}\text { Rakuten } \\
\text { Tokyo Japan /2000 }\end{array}$ & $\begin{array}{l}\text { Industry } \\
\text { WWW }\end{array}$ & 192 & $\begin{array}{r}\mathbf{1 1 8 . 1 6} \\
153.6\end{array}$ & \\
\hline 177 & $\begin{array}{c}\text { Sun } \\
\text { HPC } 10000400 \mathrm{MHz} \text { Cluster }\end{array}$ & $\begin{array}{c}\text { Telecommunication Company } \\
\text { Tokyo Japan /2000 }\end{array}$ & $\begin{array}{l}\text { Industry } \\
\text { Telecomm }\end{array}$ & 192 & $\begin{array}{r}\mathbf{1 1 8 . 1 6} \\
153.6\end{array}$ & \\
\hline 178 & $\begin{array}{c}\text { Sun } \\
\text { HPC } 10000400 \mathrm{MHz} \text { Cluster }\end{array}$ & $\begin{array}{c}\text { EDS } \\
\text { Plano USA } / 2000\end{array}$ & $\begin{array}{l}\text { Industry } \\
\text { Finance }\end{array}$ & 192 & $\begin{array}{r}\mathbf{1 1 8 . 1} \\
153.6\end{array}$ & \\
\hline 179 & $\begin{array}{c}\text { Sun } \\
\text { HPC } 10000400 \mathrm{MHz} \text { Cluster }\end{array}$ & $\begin{array}{l}\text { Telcel Radiomovil Dipsa } \\
\text { Mexico City Mexico /2000 }\end{array}$ & $\begin{array}{l}\text { Industry } \\
\text { Telecomm }\end{array}$ & 192 & $\begin{array}{r}\mathbf{1 1 8 . 1} \\
153.6\end{array}$ & \\
\hline 180 & $\begin{array}{l}\text { Cray Inc. } \\
\text { T3E }\end{array}$ & $\begin{array}{c}\text { CNRS/IDRIS } \\
\text { Orsay France /1996 }\end{array}$ & Academic & 268 & $\begin{array}{r}\mathbf{1 1 7} \\
160.8\end{array}$ & \\
\hline
\end{tabular}


TOP500 Supercomputers - Worldwide

\begin{tabular}{|c|c|c|c|c|c|c|}
\hline $\begin{array}{r}\mathbf{N} \\
\text { world }\end{array}$ & $\begin{array}{l}\text { Manufacturer } \\
\text { Computer }\end{array}$ & $\begin{array}{l}\text { Installation Site } \\
\text { Location/Year }\end{array}$ & $\begin{array}{c}\text { Field of } \\
\text { Application }\end{array}$ & $\begin{array}{c}\# \\
\text { Proc. }\end{array}$ & $\begin{array}{r}\mathbf{R}_{\max } \\
R_{\text {peak }} \\
\text { [Gflop/s] }\end{array}$ & $\begin{array}{r}N_{\max } \\
N_{1 / 2}\end{array}$ \\
\hline 181 & $\begin{array}{l}\text { Cray Inc. } \\
\text { T3E }\end{array}$ & $\begin{array}{l}\text { Government } \\
\text { USA / } 1997\end{array}$ & Classified & 268 & $\begin{array}{r}117 \\
160.8\end{array}$ & \\
\hline 182 & $\begin{array}{l}\text { Cray Inc. } \\
\text { T3E }\end{array}$ & $\begin{array}{c}\text { National Supercomputer Centre (NSC) } \\
\text { Linkoping Sweden /1997 }\end{array}$ & Academic & 268 & $\begin{array}{r}\mathbf{1 1 7} \\
160.8\end{array}$ & \\
\hline 183 & $\begin{array}{l}\text { Cray Inc. } \\
\text { T3E }\end{array}$ & $\begin{array}{l}\text { UCSD/San Diego Supercomputer Center } \\
\text { San Diego USA /1996 }\end{array}$ & Academic & 268 & $\begin{array}{r}\mathbf{1 1 7} \\
160.8\end{array}$ & \\
\hline 184 & $\begin{array}{c}\text { IBM } \\
\text { SP Power3 } 375 \mathrm{MHz}\end{array}$ & $\begin{array}{c}\text { Geco-Prakla } \\
\text { Houston USA / } 2000\end{array}$ & $\begin{array}{l}\text { Industry } \\
\text { Geophysics }\end{array}$ & 112 & $\begin{array}{r}116 \\
168\end{array}$ & \\
\hline 185 & $\begin{array}{c}\text { Hitachi } \\
\text { SR8000/16 }\end{array}$ & $\begin{array}{l}\text { HWW/Universitaet Stuttgart DLR } \\
\text { Stuttgart Germany } / 2000\end{array}$ & Industry & 16 & $\begin{array}{r}\mathbf{1 1 5} \\
128\end{array}$ & $\begin{array}{r}42928 \\
3584\end{array}$ \\
\hline 186 & $\begin{array}{l}\text { Cray Inc. } \\
\text { T3E1350 }\end{array}$ & $\begin{array}{l}\text { Phillips Petroleum Company } \\
\text { Bartlesville USA / } 2000\end{array}$ & $\begin{array}{l}\text { Industry } \\
\text { Geophysics }\end{array}$ & 132 & $\begin{array}{r}\mathbf{1 1 3 . 9} \\
178.2\end{array}$ & \\
\hline 187 & $\begin{array}{c}\text { IBM } \\
\text { SP PC604e } 332 \mathrm{MHz}\end{array}$ & $\begin{array}{c}\text { Sprint } \\
\text { USA /1999 }\end{array}$ & $\begin{array}{l}\text { Industry } \\
\text { Telecomm }\end{array}$ & 268 & $\begin{array}{r}112 \\
177\end{array}$ & \\
\hline 188 & $\begin{array}{c}\text { Sun } \\
\text { HPC } 420450 \mathrm{MHz} \text { Cluster }\end{array}$ & $\begin{array}{c}\text { University of Adelaide } \\
\text { Adelaide Australia /2000 }\end{array}$ & Academic & 160 & $\begin{array}{r}\mathbf{1 1 0 . 0 1} \\
144\end{array}$ & \\
\hline 189 & $\begin{array}{c}\text { Fujitsu } \\
\text { VPP700/56 }\end{array}$ & $\begin{array}{c}\text { Kyushu University } \\
\text { Fukuoka Japan /1996 }\end{array}$ & Academic & 56 & $\begin{array}{r}\mathbf{1 1 0} \\
123.2\end{array}$ & $\begin{array}{r}109200 \\
10752\end{array}$ \\
\hline 190 & $\begin{array}{c}\text { IBM } \\
\text { SP P2SC } 160 \mathrm{MHz}\end{array}$ & $\begin{array}{l}\text { Atomic Weapons Establishment } \\
\text { Aldermaston UK /1999 }\end{array}$ & Classified & 252 & $\begin{array}{r}\mathbf{1 0 9 . 9} \\
161.2\end{array}$ & . \\
\hline 191 & $\begin{array}{l}\text { Cray Inc. } \\
\text { T3E1200 }\end{array}$ & $\begin{array}{l}\text { National Institute for Water and Atmospheric Resea } \\
\text { Wellington New Zealand /1999 }\end{array}$ & $\begin{array}{l}\text { Research } \\
\text { Weather }\end{array}$ & 132 & $\begin{array}{r}\mathbf{1 0 9 . 3} \\
158.4\end{array}$ & . \\
\hline 192 & $\begin{array}{c}\text { IBM } \\
\text { SP PC604e } 332 \mathrm{MHz}\end{array}$ & $\begin{array}{l}\text { ThyssenKrupp Information Systems } \\
\text { Germany /2000 }\end{array}$ & $\begin{array}{l}\text { Industry } \\
\text { Mechanics }\end{array}$ & 260 & $\begin{array}{r}\mathbf{1 0 9} \\
172\end{array}$ & . \\
\hline 193 & $\begin{array}{c}\text { IBM } \\
\text { SP PC604e } 332 \mathrm{MHz}\end{array}$ & $\begin{array}{c}\text { BASF } \\
\text { Ludwigshafen Germany /1998 }\end{array}$ & $\begin{array}{l}\text { Industry } \\
\text { Chemistry }\end{array}$ & 256 & $\begin{array}{r}\mathbf{1 0 8 . 1} \\
169.9\end{array}$ & $\begin{array}{l}81460 \\
14180\end{array}$ \\
\hline 194 & $\begin{array}{c}\text { IBM } \\
\text { SP PC604e } 332 \mathrm{MHz}\end{array}$ & $\begin{array}{c}\text { Japan Adv. Inst. of Science and Technology (JAIST) } \\
\text { Hokuriku Japan /1999 }\end{array}$ & Academic & 256 & $\begin{array}{r}\mathbf{1 0 8 . 1} \\
169.9\end{array}$ & $\begin{array}{l}81460 \\
14180\end{array}$ \\
\hline 195 & $\begin{array}{c}\text { IBM } \\
\text { SP P2SC } 160 \mathrm{MHz}\end{array}$ & $\begin{array}{l}\text { Maui High-Performance Computing Center (MHPCC) } \\
\text { USA /1998 }\end{array}$ & Research & 243 & $\begin{array}{r}\mathbf{1 0 6 . 1} \\
155.52\end{array}$ & \\
\hline 196 & $\begin{array}{c}\text { Fujitsu } \\
\text { VPP700/52 }\end{array}$ & $\begin{array}{l}\text { Leibniz Rechenzentrum } \\
\text { Muenchen Germany /1998 }\end{array}$ & Academic & 52 & $\begin{array}{r}\mathbf{1 0 6} \\
114.4\end{array}$ & . \\
\hline 197 & $\begin{array}{c}\text { Sun } \\
\text { HPC } 10000400 \mathrm{MHz} \text { Cluster }\end{array}$ & $\begin{array}{l}\text { Mannesmann Mobilfunk } \\
\text { Ratingen Germany / } 2000\end{array}$ & $\begin{array}{l}\text { Industry } \\
\text { Telecomm }\end{array}$ & 168 & $\begin{array}{r}\mathbf{1 0 5 . 3 2} \\
134.4\end{array}$ & . \\
\hline 198 & $\begin{array}{c}\text { IBM } \\
\text { SP Power3 } 375 \mathrm{MHz}\end{array}$ & $\begin{array}{c}\text { TRW } \\
\text { Cleveland USA /2000 }\end{array}$ & $\begin{array}{l}\text { Industry } \\
\text { Automotive }\end{array}$ & 100 & $\begin{array}{r}104 \\
150\end{array}$ & . \\
\hline 199 & $\begin{array}{c}\text { IBM } \\
\text { SP PC604e } 332 \mathrm{MHz}\end{array}$ & $\begin{array}{l}\text { Deere and Company } \\
\text { USA /1999 }\end{array}$ & Industry & 246 & $\begin{array}{r}103 \\
163\end{array}$ & . \\
\hline 200 & $\begin{array}{c}\text { IBM } \\
\text { SP PC604e } 332 \mathrm{MHz}\end{array}$ & $\begin{array}{c}\text { Deutsche Telekom AG } \\
\text { Darmstadt Germany /1999 }\end{array}$ & $\begin{array}{l}\text { Industry } \\
\text { Telecomm }\end{array}$ & 242 & $\begin{array}{r}102 \\
160\end{array}$ & \\
\hline
\end{tabular}

Mannheim/Tennessee November 2, 2000 
Top500 Supercomputers - Worldwide

\begin{tabular}{|c|c|c|c|c|c|c|}
\hline $\begin{array}{r}\mathbf{N} \\
\text { world }\end{array}$ & $\begin{array}{l}\text { Manufacturer } \\
\text { Computer }\end{array}$ & $\begin{array}{l}\text { Installation Site } \\
\text { Location/Year }\end{array}$ & $\begin{array}{c}\text { Field of } \\
\text { Application }\end{array}$ & $\begin{array}{c}\# \\
\text { Proc. }\end{array}$ & $\begin{array}{r}\mathbf{R}_{\text {max }} \\
R_{\text {peak }} \\
\text { [Gflop/s] }\end{array}$ & $\begin{array}{r}N_{\max } \\
N_{1 / 2}\end{array}$ \\
\hline 201 & $\begin{array}{c}\text { SGI } \\
\text { ORIGIN } 2000300 \mathrm{MHz}\end{array}$ & $\begin{array}{c}\text { Centre Informatique National (CINES) } \\
\text { Montpellier France /1999 }\end{array}$ & Research & 256 & $\begin{array}{r}\mathbf{1 0 1 . 4} \\
153.6\end{array}$ & $\begin{array}{l}86400 \\
13248\end{array}$ \\
\hline 202 & $\begin{array}{c}\text { SGI } \\
\text { ORIGIN } 2000300 \mathrm{MHz}\end{array}$ & $\begin{array}{c}\text { Okazaki National Research Institute } \\
\text { Aichi Japan /2000 }\end{array}$ & Research & 256 & $\begin{array}{r}\mathbf{1 0 1 . 4} \\
153.6\end{array}$ & $\begin{array}{l}86400 \\
13248\end{array}$ \\
\hline 203 & $\begin{array}{c}\text { SGI } \\
\text { ORIGIN } 2000300 \mathrm{MHz}\end{array}$ & $\begin{array}{c}\text { Tokyo Institute of Technology } \\
\text { Tokyo Japan /2000 }\end{array}$ & Academic & 256 & $\begin{array}{r}\mathbf{1 0 1 . 4} \\
153.6\end{array}$ & $\begin{array}{l}86400 \\
13248\end{array}$ \\
\hline 204 & $\begin{array}{c}\text { SGI } \\
\text { ORIGIN } 2000250 \mathrm{MHz}\end{array}$ & $\begin{array}{c}\text { NASA/Ames Research Center/NAS } \\
\text { Mountain View USA /1998 }\end{array}$ & $\begin{array}{l}\text { Research } \\
\text { Aerospace }\end{array}$ & 256 & $\begin{array}{r}\mathbf{1 0 1 . 4} \\
128\end{array}$ & $\begin{array}{l}86400 \\
13248\end{array}$ \\
\hline 205 & $\begin{array}{c}\text { Cray Inc. } \\
\text { T3D MC1024-8 }\end{array}$ & $\begin{array}{l}\text { Government } \\
\text { USA / } 1994\end{array}$ & Classified & 1024 & $\begin{array}{r}\mathbf{1 0 0 . 5} \\
152\end{array}$ & $\begin{array}{l}81920 \\
10224\end{array}$ \\
\hline 206 & $\begin{array}{c}\text { IBM } \\
\text { SP Power3 } 375 \mathrm{MHz} \\
\end{array}$ & $\begin{array}{c}\text { BRS STF } \\
\text { USA / } 2000\end{array}$ & Industry & 96 & $\begin{array}{r}\mathbf{1 0 0} \\
144\end{array}$ & \\
\hline 207 & $\begin{array}{c}\text { IBM } \\
\text { SP Power3 } 375 \mathrm{MHz}\end{array}$ & $\begin{array}{c}\text { DaimlerChrysler } \\
\text { Stuttgart Germany /2000 }\end{array}$ & $\begin{array}{l}\text { Industry } \\
\text { Automotive }\end{array}$ & 96 & $\begin{array}{r}\mathbf{1 0 0} \\
144\end{array}$ & \\
\hline 208 & $\begin{array}{c}\text { IBM } \\
\text { SP Power3 } 375 \mathrm{MHz}\end{array}$ & $\begin{array}{c}\text { Telecom Denmark (Danadata) } \\
\text { Denmark /2000 }\end{array}$ & $\begin{array}{l}\text { Industry } \\
\text { Telecomm }\end{array}$ & 96 & $\begin{array}{r}100 \\
144\end{array}$ & \\
\hline 209 & $\begin{array}{c}\text { IBM } \\
\text { SP Power3 } 375 \mathrm{MHz}\end{array}$ & $\begin{array}{c}\text { Telekom Austria } \\
\text { Austria /2000 }\end{array}$ & $\begin{array}{l}\text { Industry } \\
\text { Telecomm }\end{array}$ & 96 & $\begin{array}{r}\mathbf{1 0 0} \\
144\end{array}$ & \\
\hline 210 & $\begin{array}{c}\text { Compaq } \\
\text { ALiCE EV67 } 616 \mathrm{MHz}\end{array}$ & $\begin{array}{c}\text { Universitaet Wuppertal } \\
\text { Wuppertal Germany /2000 }\end{array}$ & Academic & 128 & $\begin{array}{r}99.2 \\
157\end{array}$ & $\begin{array}{l}57024 \\
15488\end{array}$ \\
\hline 211 & \begin{tabular}{|c|} 
SGI \\
ORIGIN $2000250 / 300 \mathrm{MHz}-$ Eth-Cluster
\end{tabular} & $\begin{array}{c}\text { SARA (Stichting Academisch Rekencentrum) } \\
\text { Amsterdam Netherlands } / 2000\end{array}$ & Academic & 256 & $\begin{array}{r}98.8 \\
140 \\
\end{array}$ & 81920 \\
\hline 212 & $\begin{array}{c}\text { IBM } \\
\text { SP Power3 } 375 \mathrm{MHz}\end{array}$ & $\begin{array}{c}\text { FT USEI } \\
\text { France } / 2000\end{array}$ & $\begin{array}{l}\text { Industry } \\
\text { Telecomm }\end{array}$ & 94 & $\begin{array}{r}98.2 \\
141\end{array}$ & \\
\hline 213 & $\begin{array}{c}\text { IBM } \\
\text { SP PC604e } 332 \mathrm{MHz}\end{array}$ & $\begin{array}{c}\text { Chase Manhattan } \\
\text { New York USA /1999 }\end{array}$ & $\begin{array}{l}\text { Industry } \\
\text { Finance }\end{array}$ & 232 & $\begin{array}{r}98 \\
153\end{array}$ & \\
\hline 214 & $\begin{array}{l}\text { Fujitsu } \\
\text { VPP700/48E }\end{array}$ & $\begin{array}{c}\text { ECMWF } \\
\text { Reading UK /1998 }\end{array}$ & $\begin{array}{l}\text { Research } \\
\text { Weather }\end{array}$ & 48 & $\begin{array}{l}\mathbf{9 7 . 5} \\
115.2\end{array}$ & \\
\hline 215 & $\begin{array}{c}\text { Self-made } \\
\text { Kepler PIII } 650 \mathrm{MHz}\end{array}$ & $\begin{array}{c}\text { Universitaet Tuebingen } \\
\text { Tuebingen Germany /2000 }\end{array}$ & Academic & 196 & $\begin{array}{r}\mathbf{9 6 . 2} \\
127 \\
\end{array}$ & $\begin{array}{r}109760 \\
12320\end{array}$ \\
\hline 216 & $\begin{array}{c}\text { IBM } \\
\text { SP PC604e } 332 \mathrm{MHz}\end{array}$ & $\begin{array}{c}\text { ThyssenKrupp Information Systems } \\
\text { Germany /2000 }\end{array}$ & $\begin{array}{l}\text { Industry } \\
\text { Mechanics }\end{array}$ & 226 & $\begin{array}{r}95.4 \\
149\end{array}$ & \\
\hline 217 & $\begin{array}{c}\text { IBM } \\
\text { SP P2SC } 135 \mathrm{MHz}\end{array}$ & $\begin{array}{c}\text { ERDC MSRC } \\
\text { Vicksburg USA /1997 }\end{array}$ & Research & 256 & $\begin{array}{r}\mathbf{9 4 . 1 9} \\
138.24\end{array}$ & \\
\hline 218 & $\begin{array}{c}\text { IBM } \\
\text { SP P2SC } 135 \mathrm{MHz}\end{array}$ & $\begin{array}{c}\text { Wright-Patterson Air Force Base/DoD ASC } \\
\text { USA / } 1997\end{array}$ & Research & 256 & $\begin{array}{r}\mathbf{9 4 . 1 9} \\
138.24\end{array}$ & \\
\hline 219 & $\begin{array}{c}\text { SGI } \\
\text { ORIGIN } 2000250 \mathrm{MHz} \text { - Eth-Cluster }\end{array}$ & $\begin{array}{l}\text { Government } \\
\text { USA / } 1999\end{array}$ & Classified & 256 & $\begin{array}{r}92.99 \\
128 \\
\end{array}$ & $\begin{array}{l}81920 \\
81920\end{array}$ \\
\hline 220 & $\begin{array}{c}\text { SGI } \\
\text { ORIGIN } 2000250 \mathrm{MHz} \text { - Eth-Cluster }\end{array}$ & $\begin{array}{c}\text { Government } \\
\text { USA / } 1999\end{array}$ & Classified & 256 & $\begin{array}{r}92.99 \\
128\end{array}$ & $\begin{array}{l}81920 \\
81920\end{array}$ \\
\hline
\end{tabular}

Mannheim/Tennessee November 2, 2000 
ToP500 Supercomputers - Worldwide

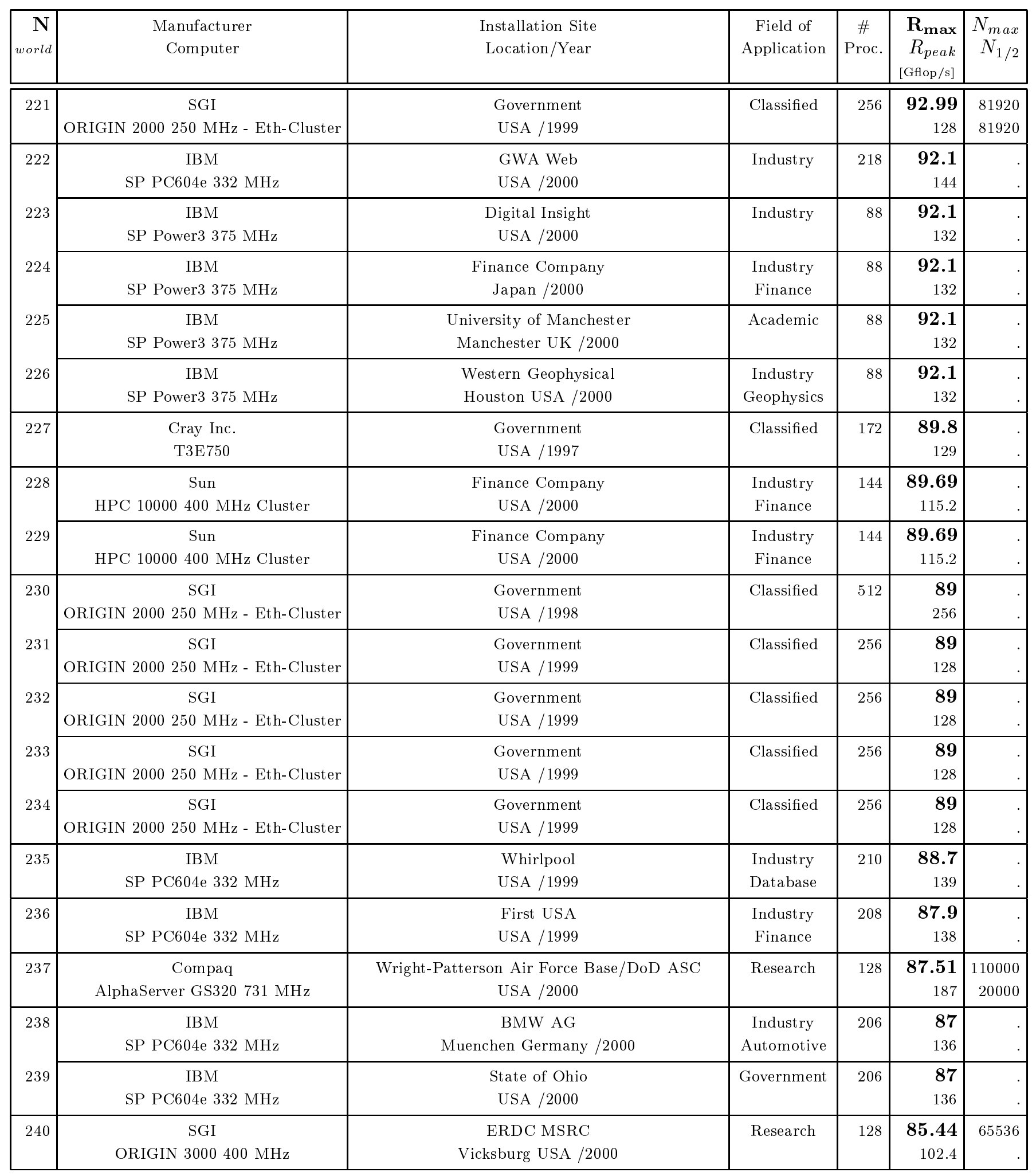

Mannheim/Tennessee November 2, 2000 
Top500 Supercomputers - Worldwide

\begin{tabular}{|c|c|c|c|c|c|c|}
\hline $\begin{array}{r}\mathbf{N} \\
\text { world }\end{array}$ & $\begin{array}{l}\text { Manufacturer } \\
\text { Computer }\end{array}$ & $\begin{array}{l}\text { Installation Site } \\
\text { Location/Year }\end{array}$ & $\begin{array}{c}\text { Field of } \\
\text { Application }\end{array}$ & $\begin{array}{c}\# \\
\text { Proc. }\end{array}$ & $\begin{array}{r}\mathbf{R}_{\max } \\
R_{\text {peak }} \\
{[\text { Gflop } / \mathrm{s}]}\end{array}$ & $\begin{array}{r}N_{\max } \\
N_{1 / 2}\end{array}$ \\
\hline 241 & $\begin{array}{c}\text { SGI } \\
\text { ORIGIN } 3000400 \mathrm{MHz}\end{array}$ & $\begin{array}{c}\text { ERDC MSRC } \\
\text { Vicksburg USA / } 2000\end{array}$ & Research & 128 & $\begin{array}{r}85.44 \\
102.4\end{array}$ & 65536 \\
\hline 242 & $\begin{array}{c}\text { SGI } \\
\text { ORIGIN } 3000400 \mathrm{MHz}\end{array}$ & $\begin{array}{c}\text { SARA (Stichting Academisch Rekencentrum) } \\
\text { Amsterdam Netherlands } / 2000\end{array}$ & Academic & 128 & $\begin{array}{r}85.44 \\
102.4\end{array}$ & 65536 \\
\hline 243 & $\begin{array}{c}\text { SGI } \\
\text { ORIGIN } 3000400 \mathrm{MHz}\end{array}$ & $\begin{array}{c}\text { SARA (Stichting Academisch Rekencentrum) } \\
\text { Amsterdam Netherlands } / 2000\end{array}$ & Academic & 128 & $\begin{array}{r}85.44 \\
102.4\end{array}$ & 65536 \\
\hline 244 & $\begin{array}{c}\text { SGI } \\
\text { ORIGIN } 3000400 \mathrm{MHz}\end{array}$ & $\begin{array}{c}\text { SARA (Stichting Academisch Rekencentrum) } \\
\text { Amsterdam Netherlands } / 2000\end{array}$ & Academic & 128 & $\begin{array}{r}85.44 \\
102.4\end{array}$ & 65536 \\
\hline 245 & $\begin{array}{c}\text { SGI } \\
\text { ORIGIN } 3000400 \mathrm{MHz}\end{array}$ & $\begin{array}{c}\text { SARA (Stichting Academisch Rekencentrum) } \\
\text { Amsterdam Netherlands } / 2000\end{array}$ & Academic & 128 & $\begin{array}{r}85.44 \\
102.4\end{array}$ & 65536 \\
\hline 246 & $\begin{array}{c}\text { SGI } \\
\text { ORIGIN } 3000400 \mathrm{MHz}\end{array}$ & $\begin{array}{c}\text { SARA (Stichting Academisch Rekencentrum) } \\
\text { Amsterdam Netherlands } / 2000\end{array}$ & Academic & 128 & $\begin{array}{r}85.44 \\
102.4\end{array}$ & 65536 \\
\hline 247 & $\begin{array}{c}\text { SGI } \\
\text { ORIGIN } 3000400 \mathrm{MHz}\end{array}$ & $\begin{array}{c}\text { SARA (Stichting Academisch Rekencentrum) } \\
\text { Amsterdam Netherlands } / 2000\end{array}$ & Academic & 128 & $\begin{array}{r}85.44 \\
102.4\end{array}$ & 65536 \\
\hline 248 & $\begin{array}{c}\text { SGI } \\
\text { ORIGIN } 3000400 \mathrm{MHz}\end{array}$ & $\begin{array}{c}\text { SARA (Stichting Academisch Rekencentrum) } \\
\text { Amsterdam Netherlands } / 2000\end{array}$ & Academic & 128 & $\begin{array}{r}85.44 \\
102.4\end{array}$ & 65536 \\
\hline 249 & $\begin{array}{c}\text { SGI } \\
\text { ORIGIN } 3000400 \mathrm{MHz}\end{array}$ & $\begin{array}{c}\text { SARA (Stichting Academisch Rekencentrum) } \\
\text { Amsterdam Netherlands } / 2000\end{array}$ & Academic & 128 & $\begin{array}{r}85.44 \\
102.4\end{array}$ & 65536 \\
\hline 250 & $\begin{array}{c}\text { SGI } \\
\text { ORIGIN } 3000400 \mathrm{MHz}\end{array}$ & $\begin{array}{l}\text { Silicon Graphics } \\
\text { Mountain View USA /2000 }\end{array}$ & Vendor & 128 & $\begin{array}{r}85.44 \\
102.4\end{array}$ & 65536 \\
\hline 251 & $\begin{array}{c}\text { SGI } \\
\text { ORIGIN } 3000400 \mathrm{MHz}\end{array}$ & $\begin{array}{l}\text { Silicon Graphics } \\
\text { Mountain View USA /2000 }\end{array}$ & Vendor & 128 & $\begin{array}{r}85.44 \\
102.4\end{array}$ & 65536 \\
\hline 252 & $\begin{array}{c}\text { SGI } \\
\text { ORIGIN } 3000400 \mathrm{MHz}\end{array}$ & $\begin{array}{l}\text { Silicon Graphics } \\
\text { Mountain View USA / } 2000\end{array}$ & Vendor & 128 & $\begin{array}{r}\mathbf{8 5 . 4 4} \\
102.4\end{array}$ & 65536 \\
\hline 253 & $\begin{array}{c}\text { SGI } \\
\text { ORIGIN } 3000400 \mathrm{MHz}\end{array}$ & $\begin{array}{c}\text { US Army Research Laboratory (ARL) } \\
\text { Aberdeen USA / } 2000\end{array}$ & Research & 128 & $\begin{array}{r}\mathbf{8 5 . 4 4} \\
102.4\end{array}$ & 65536 \\
\hline 254 & $\begin{array}{c}\text { SGI } \\
\text { ORIGIN } 3000400 \mathrm{MHz}\end{array}$ & $\begin{array}{c}\text { US Army Research Laboratory (ARL) } \\
\text { Aberdeen USA / } 2000\end{array}$ & Research & 128 & $\begin{array}{r}85.44 \\
102.4\end{array}$ & 65536 \\
\hline 255 & $\begin{array}{c}\text { SGI } \\
\text { ORIGIN } 3000400 \mathrm{MHz}\end{array}$ & $\begin{array}{c}\text { US Army Research Laboratory (ARL) } \\
\text { Aberdeen USA / } 2000\end{array}$ & Research & 128 & $\begin{array}{r}85.44 \\
102.4\end{array}$ & 65536 \\
\hline 256 & $\begin{array}{c}\text { SGI } \\
\text { ORIGIN } 3000400 \mathrm{MHz}\end{array}$ & $\begin{array}{c}\text { US Army Research Laboratory (ARL) } \\
\text { Aberdeen USA / } 2000\end{array}$ & Research & 128 & $\begin{array}{r}85.44 \\
102.4\end{array}$ & 65536 \\
\hline 257 & $\begin{array}{c}\text { SGI } \\
\text { ORIGIN } 3000400 \mathrm{MHz}\end{array}$ & $\begin{array}{c}\text { US Army Research Laboratory (ARL) } \\
\text { Aberdeen USA / } 2000\end{array}$ & Research & 128 & $\begin{array}{r}85.44 \\
102.4\end{array}$ & 65536 \\
\hline 258 & $\begin{array}{c}\text { SGI } \\
\text { ORIGIN } 3000400 \mathrm{MHz}\end{array}$ & $\begin{array}{c}\text { US Army Research Laboratory (ARL) } \\
\text { Aberdeen USA / } 2000\end{array}$ & Research & 128 & $\begin{array}{r}85.44 \\
102.4\end{array}$ & 65536 \\
\hline 259 & $\begin{array}{c}\text { SGI } \\
\text { ORIGIN } 3000400 \mathrm{MHz}\end{array}$ & $\begin{array}{l}\text { University of Leicester } \\
\text { Leicester UK / } 2000\end{array}$ & Academic & 128 & $\begin{array}{r}85.44 \\
102.4\end{array}$ & 65536 \\
\hline 260 & $\begin{array}{c}\text { IBM } \\
\text { SP PC604e } 332 \mathrm{MHz}\end{array}$ & $\begin{array}{c}\text { Aetna Life Insurance } \\
\text { Middletown USA / } 1999\end{array}$ & $\begin{array}{l}\text { Industry } \\
\text { Database }\end{array}$ & 200 & $\begin{array}{r}84.5 \\
132\end{array}$ & \\
\hline
\end{tabular}

Mannheim/Tennessee November 2, 2000 
ToP500 Supercomputers - Worldwide

\begin{tabular}{|c|c|c|c|c|c|c|}
\hline $\begin{array}{r}\mathbf{N} \\
\text { world }\end{array}$ & $\begin{array}{l}\text { Manufacturer } \\
\text { Computer }\end{array}$ & $\begin{array}{l}\text { Installation Site } \\
\text { Location/Year }\end{array}$ & $\begin{array}{c}\text { Field of } \\
\text { Application }\end{array}$ & $\begin{array}{c}\# \\
\text { Proc. }\end{array}$ & $\begin{array}{r}\mathbf{R}_{\text {max }} \\
R_{\text {peak }} \\
\text { [Gflop/s] }\end{array}$ & $\begin{array}{r}N_{\max } \\
N_{1 / 2}\end{array}$ \\
\hline 261 & $\begin{array}{c}\text { IBM } \\
\text { SP PC604e } 332 \mathrm{MHz}\end{array}$ & $\begin{array}{c}\text { Prudential Insurance } \\
\text { USA / } 1999\end{array}$ & $\begin{array}{l}\text { Industry } \\
\text { Finance }\end{array}$ & 200 & $\begin{array}{r}84.5 \\
132\end{array}$ & \\
\hline 262 & $\begin{array}{c}\text { IBM } \\
\text { SP PC604e } 332 \mathrm{MHz}\end{array}$ & $\begin{array}{c}\text { Sprint } \\
\text { USA / } 1999\end{array}$ & $\begin{array}{l}\text { Industry } \\
\text { Telecomm }\end{array}$ & 200 & $\begin{array}{r}84.5 \\
132\end{array}$ & \\
\hline 263 & $\begin{array}{c}\text { IBM } \\
\text { SP PC604e } 332 \mathrm{MHz}\end{array}$ & $\begin{array}{c}\text { Sprint } \\
\text { USA /1999 }\end{array}$ & $\begin{array}{l}\text { Industry } \\
\text { Telecomm }\end{array}$ & 200 & $\begin{array}{r}84.5 \\
132\end{array}$ & . \\
\hline 264 & $\begin{array}{c}\text { IBM } \\
\text { SP Power3 } 375 \mathrm{MHz}\end{array}$ & $\begin{array}{c}\text { Aetna Life Insurance } \\
\text { Middletown USA / } 2000\end{array}$ & $\begin{array}{l}\text { Industry } \\
\text { Database }\end{array}$ & 80 & $\begin{array}{r}84 \\
120\end{array}$ & \\
\hline 265 & $\begin{array}{c}\text { IBM } \\
\text { SP Power3 } 375 \mathrm{MHz}\end{array}$ & $\begin{array}{l}\text { Credit Suisse } \\
\text { Zurich Switzerland /2000 }\end{array}$ & $\begin{array}{l}\text { Industry } \\
\text { Finance }\end{array}$ & 80 & $\begin{array}{r}84 \\
120\end{array}$ & \\
\hline 266 & $\begin{array}{c}\text { IBM } \\
\text { SP Power3 } 375 \mathrm{MHz}\end{array}$ & $\begin{array}{c}\text { Financial Company } \\
\text { Japan } / 2000\end{array}$ & $\begin{array}{l}\text { Industry } \\
\text { Finance }\end{array}$ & 80 & $\begin{array}{r}84 \\
120\end{array}$ & \\
\hline 267 & $\begin{array}{c}\text { IBM } \\
\text { SP Power3 } 375 \mathrm{MHz}\end{array}$ & $\begin{array}{c}\text { Kroger } \\
\text { USA / } 2000\end{array}$ & Industry & 80 & $\begin{array}{r}84 \\
120\end{array}$ & \\
\hline 268 & $\begin{array}{c}\text { IBM } \\
\text { SP Power3 } 375 \mathrm{MHz}\end{array}$ & $\begin{array}{c}\text { Manufacturing Company } \\
\text { Japan } / 2000\end{array}$ & $\begin{array}{c}\text { Industry } \\
\text { Manufacturing }\end{array}$ & 80 & $\begin{array}{r}84 \\
120\end{array}$ & \\
\hline 269 & $\begin{array}{c}\text { IBM } \\
\text { SP Power3 } 375 \mathrm{MHz}\end{array}$ & $\begin{array}{c}\text { Philips Lighting } \\
\text { USA / } 2000\end{array}$ & Industry & 80 & $\begin{array}{r}84 \\
120\end{array}$ & \\
\hline 270 & $\begin{array}{c}\text { IBM } \\
\text { SP Power3 } 375 \mathrm{MHz}\end{array}$ & $\begin{array}{c}\text { Postes Telecom } \\
\text { Belgium /2000 }\end{array}$ & $\begin{array}{l}\text { Industry } \\
\text { Telecomm }\end{array}$ & 80 & $\begin{array}{r}84 \\
120\end{array}$ & \\
\hline 271 & $\begin{array}{c}\text { IBM } \\
\text { SP Power3 } 375 \mathrm{MHz}\end{array}$ & $\begin{array}{c}\text { Volvo } \\
\text { Gothenberg Sweden } / 2000\end{array}$ & $\begin{array}{l}\text { Industry } \\
\text { Automotive }\end{array}$ & 80 & $\begin{array}{r}84 \\
120\end{array}$ & \\
\hline 272 & $\begin{array}{c}\text { IBM } \\
\text { SP PC604e } 332 \mathrm{MHz}\end{array}$ & $\begin{array}{c}\text { ULC } \\
\text { USA / } 2000\end{array}$ & Industry & 196 & $\begin{array}{r}82.8 \\
130\end{array}$ & \\
\hline 273 & $\begin{array}{l}\text { Cray Inc. } \\
\text { T3E900 }\end{array}$ & $\begin{array}{c}\text { KIST/System Engineering Research Institute (SSC) } \\
\text { Korea /1997 }\end{array}$ & $\begin{array}{l}\text { Industry } \\
\text { In.Pr. Service }\end{array}$ & 132 & $\begin{array}{l}82.1 \\
118.8\end{array}$ & \\
\hline 274 & $\begin{array}{c}\text { Cray Inc. } \\
\text { T3E900 }\end{array}$ & $\begin{array}{l}\text { NOAA/Geophysical Fluid Dynamics Laboratory (GFDL) } \\
\text { Princeton USA / } 1997\end{array}$ & $\begin{array}{l}\text { Research } \\
\text { Weather }\end{array}$ & 132 & $\begin{array}{l}\mathbf{8 2 . 1} \\
118.8\end{array}$ & \\
\hline 275 & $\begin{array}{c}\text { IBM } \\
\text { SP PC604e } 332 \mathrm{MHz}\end{array}$ & $\begin{array}{l}\text { NAC RE International } \\
\text { USA / } 2000\end{array}$ & Industry & 194 & $\begin{array}{r}82 \\
128\end{array}$ & \\
\hline 276 & $\begin{array}{c}\text { SGI } \\
\text { ORIGIN } 2000400 \mathrm{MHz}\end{array}$ & $\begin{array}{l}\text { Silicon Graphics } \\
\text { Eagan USA / } 2000\end{array}$ & Vendor & 128 & $\begin{array}{r}81.76 \\
102.4\end{array}$ & 65536 \\
\hline 277 & $\begin{array}{c}\text { SGI } \\
\text { ORIGIN } 2000400 \mathrm{MHz}\end{array}$ & $\begin{array}{l}\text { Silicon Graphics } \\
\text { Eagan USA / } 2000\end{array}$ & Vendor & 128 & $\begin{array}{r}81.76 \\
102.4\end{array}$ & 65536 \\
\hline 278 & $\begin{array}{c}\text { SGI } \\
\text { ORIGIN } 2000400 \mathrm{MHz}\end{array}$ & $\begin{array}{l}\text { University of Manchester } \\
\text { Manchester UK /2000 }\end{array}$ & Academic & 128 & $\begin{array}{r}81.76 \\
102.4\end{array}$ & 65536 \\
\hline 279 & $\begin{array}{l}\text { Cray Inc. } \\
\text { T3E }\end{array}$ & $\begin{array}{c}\text { Commissariat a l'Energie Atomique (CEA) } \\
\text { Bruyeres France /1997 }\end{array}$ & Research & 188 & $\begin{array}{l}81.3 \\
112.8\end{array}$ & \\
\hline 280 & $\begin{array}{c}\text { IBM } \\
\text { SP PC604e } 332 \mathrm{MHz}\end{array}$ & $\begin{array}{c}\text { Autozone } \\
\text { Memphis USA /1999 }\end{array}$ & $\begin{array}{l}\text { Industry } \\
\text { Database }\end{array}$ & 192 & $\begin{array}{r}81.1 \\
127\end{array}$ & \\
\hline
\end{tabular}

Mannheim/Tennessee November 2, 2000 
TOP500 Supercomputers - Worldwide

\begin{tabular}{|c|c|c|c|c|c|c|}
\hline $\begin{array}{r}\mathbf{N} \\
\text { world }\end{array}$ & $\begin{array}{l}\text { Manufacturer } \\
\text { Computer }\end{array}$ & $\begin{array}{l}\text { Installation Site } \\
\text { Location/Year }\end{array}$ & $\begin{array}{c}\text { Field of } \\
\text { Application }\end{array}$ & $\begin{array}{c}\# \\
\text { Proc. }\end{array}$ & $\begin{array}{r}\mathbf{R}_{\max } \\
R_{\text {peak }} \\
{[\mathrm{Gflop} / \mathrm{s}]}\end{array}$ & $\begin{array}{r}N_{\max } \\
N_{1 / 2}\end{array}$ \\
\hline 281 & $\begin{array}{c}\text { IBM } \\
\text { SP Power3 } 222 \mathrm{MHz}\end{array}$ & $\begin{array}{c}\text { Dupont } \\
\text { USA / } 2000\end{array}$ & Industry & 128 & $\begin{array}{r}80.83 \\
113.6\end{array}$ & $\begin{array}{l}76000 \\
15000\end{array}$ \\
\hline 282 & $\begin{array}{c}\text { Sun } \\
\text { HPC } 10000400 \mathrm{MHz} \text { Cluster }\end{array}$ & $\begin{array}{c}\text { Aerotek } \\
\text { Hanover USA /1999 }\end{array}$ & $\begin{array}{c}\text { Industry } \\
\text { Manufacturing }\end{array}$ & 128 & $\begin{array}{r}\mathbf{8 0 . 1} \\
102.4\end{array}$ & $\begin{array}{l}57120 \\
10752\end{array}$ \\
\hline 283 & $\begin{array}{c}\text { Sun } \\
\text { HPC } 10000400 \mathrm{MHz} \text { Cluster }\end{array}$ & $\begin{array}{l}\text { Ameritrade Inc. } \\
\text { Omaha USA / } 2000\end{array}$ & $\begin{array}{l}\text { Industry } \\
\text { Finance }\end{array}$ & 128 & $\begin{array}{l}\mathbf{8 0 . 1} \\
102.4\end{array}$ & $\begin{array}{l}57120 \\
10752\end{array}$ \\
\hline 284 & $\begin{array}{c}\text { Sun } \\
\text { HPC } 10000400 \mathrm{MHz} \text { Cluster }\end{array}$ & $\begin{array}{c}\text { Baker Hughes } \\
\text { Houston USA / } 1999\end{array}$ & $\begin{array}{l}\text { Industry } \\
\text { Geophysics }\end{array}$ & 128 & $\begin{array}{r}80.1 \\
102.4\end{array}$ & $\begin{array}{l}57120 \\
10752\end{array}$ \\
\hline 285 & \begin{tabular}{|c} 
Sun \\
HPC $10000400 \mathrm{MHz}$ Cluster
\end{tabular} & $\begin{array}{l}\text { Clearstream Services } \\
\text { Grande Duchesse Luxembourg /2000 }\end{array}$ & $\begin{array}{l}\text { Industry } \\
\text { Finance }\end{array}$ & 128 & $\begin{array}{r}80.1 \\
102.4\end{array}$ & $\begin{array}{l}57120 \\
10752\end{array}$ \\
\hline 286 & $\begin{array}{c}\text { Sun } \\
\text { HPC } 10000400 \mathrm{MHz} \text { Cluster }\end{array}$ & $\begin{array}{c}\text { Deutsche Telekom AG } \\
\text { Bamberg Germany /2000 }\end{array}$ & $\begin{array}{l}\text { Industry } \\
\text { Telecomm }\end{array}$ & 128 & $\begin{array}{r}80.1 \\
102.4\end{array}$ & $\begin{array}{l}57120 \\
10752\end{array}$ \\
\hline 287 & $\begin{array}{c}\text { Sun } \\
\text { HPC } 10000400 \mathrm{MHz} \text { Cluster }\end{array}$ & $\begin{array}{c}\text { E-commerce } \\
\text { Santa Clara USA /2000 }\end{array}$ & $\begin{array}{l}\text { Industry } \\
\text { WWW }\end{array}$ & 128 & $\begin{array}{l}\mathbf{8 0 . 1} \\
102.4\end{array}$ & $\begin{array}{l}57120 \\
10752\end{array}$ \\
\hline 288 & $\begin{array}{c}\text { Sun } \\
\text { HPC } 10000400 \mathrm{MHz} \text { Cluster }\end{array}$ & $\begin{array}{c}\text { Internet Service Provider } \\
\text { Italy } / 2000\end{array}$ & $\begin{array}{l}\text { Industry } \\
\text { WWW }\end{array}$ & 128 & $\begin{array}{r}80.1 \\
102.4\end{array}$ & $\begin{array}{l}57120 \\
10752\end{array}$ \\
\hline 289 & $\begin{array}{c}\text { Sun } \\
\text { HPC } 10000400 \mathrm{MHz} \text { Cluster }\end{array}$ & $\begin{array}{c}\text { Lexis Nexis } \\
\text { Miamisburg USA /2000 }\end{array}$ & $\begin{array}{l}\text { Industry } \\
\text { Infor. Service }\end{array}$ & 128 & $\begin{array}{l}\mathbf{8 0 . 1} \\
102.4\end{array}$ & $\begin{array}{l}57120 \\
10752\end{array}$ \\
\hline 290 & $\begin{array}{c}\text { Sun } \\
\text { HPC } 10000400 \mathrm{MHz} \text { Cluster }\end{array}$ & $\begin{array}{c}\text { Motorola } \\
\text { Schaumburg USA /2000 }\end{array}$ & $\begin{array}{l}\text { Industry } \\
\text { Electronics }\end{array}$ & 128 & $\begin{array}{l}\mathbf{8 0 . 1} \\
102.4\end{array}$ & $\begin{array}{l}57120 \\
10752\end{array}$ \\
\hline 291 & $\begin{array}{c}\text { Sun } \\
\text { HPC } 10000400 \mathrm{MHz} \text { Cluster }\end{array}$ & $\begin{array}{c}\text { OfficeMax } \\
\text { Shaker Heights USA /1999 }\end{array}$ & $\begin{array}{l}\text { Industry } \\
\text { Database }\end{array}$ & 128 & $\begin{array}{r}\mathbf{8 0 . 1} \\
102.4\end{array}$ & $\begin{array}{l}57120 \\
10752\end{array}$ \\
\hline 292 & $\begin{array}{c}\text { Sun } \\
\text { HPC } 10000400 \mathrm{MHz} \text { Cluster }\end{array}$ & $\begin{array}{c}\text { RandomHouse } \\
\text { Westminster USA /1999 }\end{array}$ & $\begin{array}{l}\text { Industry } \\
\text { Database }\end{array}$ & 128 & $\begin{array}{l}\mathbf{8 0 . 1} \\
102.4\end{array}$ & $\begin{array}{l}57120 \\
10752\end{array}$ \\
\hline 293 & $\begin{array}{c}\text { Sun } \\
\text { HPC } 10000400 \mathrm{MHz} \text { Cluster }\end{array}$ & $\begin{array}{c}\text { Rutgers University } \\
\text { Piscataway USA / } 1999\end{array}$ & Academic & 128 & $\begin{array}{r}\mathbf{8 0 . 1} \\
102.4\end{array}$ & $\begin{array}{l}57120 \\
10752\end{array}$ \\
\hline 294 & \begin{tabular}{|c} 
Sun \\
HPC $10000400 \mathrm{MHz}$ Cluster
\end{tabular} & $\begin{array}{c}\text { Stanford University/High Energy Physics } \\
\text { Palo Alto USA / } 2000\end{array}$ & Academic & 128 & $\begin{array}{l}80.1 \\
102.4\end{array}$ & $\begin{array}{l}57120 \\
10752\end{array}$ \\
\hline 295 & $\begin{array}{c}\text { Sun } \\
\text { HPC } 10000400 \mathrm{MHz} \text { Cluster }\end{array}$ & $\begin{array}{l}\text { Telecommunication Company } \\
\text { Sydney Australia / } 2000\end{array}$ & $\begin{array}{l}\text { Industry } \\
\text { Telecomm }\end{array}$ & 128 & $\begin{array}{r}80.1 \\
102.4\end{array}$ & $\begin{array}{l}57120 \\
10752\end{array}$ \\
\hline 296 & $\begin{array}{c}\text { Sun } \\
\text { HPC } 10000400 \mathrm{MHz} \text { Cluster }\end{array}$ & $\begin{array}{c}\text { Telemar } \\
\text { Brazil /2000 }\end{array}$ & $\begin{array}{l}\text { Industry } \\
\text { Telecomm }\end{array}$ & 128 & $\begin{array}{l}\mathbf{8 0 . 1} \\
102.4\end{array}$ & $\begin{array}{l}57120 \\
10752\end{array}$ \\
\hline 297 & $\begin{array}{c}\text { Sun } \\
\text { HPC } 10000400 \mathrm{MHz} \text { Cluster }\end{array}$ & $\begin{array}{l}\text { W.W. Grainger } \\
\text { Niles USA / } 2000\end{array}$ & $\begin{array}{l}\text { Industry } \\
\text { Database }\end{array}$ & 128 & $\begin{array}{l}\mathbf{8 0 . 1} \\
102.4\end{array}$ & $\begin{array}{l}57120 \\
10752\end{array}$ \\
\hline 298 & $\begin{array}{c}\text { Sun } \\
\text { HPC } 10000400 \mathrm{MHz} \text { Cluster }\end{array}$ & $\begin{array}{c}\text { eBay } \\
\text { Santa Clara USA /2000 }\end{array}$ & $\begin{array}{l}\text { Industry } \\
\text { WWW }\end{array}$ & 128 & $\begin{array}{r}80.1 \\
102.4\end{array}$ & $\begin{array}{l}57120 \\
10752\end{array}$ \\
\hline 299 & $\begin{array}{c}\text { Sun } \\
\text { HPC } 10000400 \mathrm{MHz} \text { Cluster }\end{array}$ & $\begin{array}{c}\text { Automotive } \\
\text { Acheres France /2000 }\end{array}$ & $\begin{array}{l}\text { Industry } \\
\text { Automotive }\end{array}$ & 128 & $\begin{array}{l}80.1 \\
102.4\end{array}$ & $\begin{array}{l}57120 \\
10752\end{array}$ \\
\hline 300 & $\begin{array}{c}\text { Sun } \\
\text { HPC } 10000400 \mathrm{MHz} \text { Cluster }\end{array}$ & $\begin{array}{c}\text { Bank } \\
\text { London UK /2000 }\end{array}$ & $\begin{array}{l}\text { Industry } \\
\text { Finance }\end{array}$ & 128 & $\begin{array}{l}\mathbf{8 0 . 1} \\
102.4\end{array}$ & $\begin{array}{l}57120 \\
10752\end{array}$ \\
\hline
\end{tabular}

Mannheim/Tennessee November 2, 2000 
Top500 Supercomputers - Worldwide

\begin{tabular}{|c|c|c|c|c|c|c|}
\hline $\begin{array}{r}\mathbf{N} \\
\text { world }\end{array}$ & $\begin{array}{l}\text { Manufacturer } \\
\text { Computer }\end{array}$ & $\begin{array}{l}\text { Installation Site } \\
\text { Location/Year }\end{array}$ & $\begin{array}{c}\text { Field of } \\
\text { Application }\end{array}$ & $\begin{array}{c}\# \\
\text { Proc. }\end{array}$ & $\begin{array}{r}\mathbf{R}_{\max } \\
R_{\text {peak }} \\
{[\text { Gflop/s] }}\end{array}$ & $\begin{array}{r}N_{\max } \\
N_{1 / 2}\end{array}$ \\
\hline 301 & $\begin{array}{c}\text { Sun } \\
\text { HPC } 10000400 \mathrm{MHz} \text { Cluster }\end{array}$ & $\begin{array}{c}\text { BellSouth } \\
\text { Birmingham USA /2000 }\end{array}$ & $\begin{array}{l}\text { Industry } \\
\text { Telecomm }\end{array}$ & 128 & $\begin{array}{r}\mathbf{8 0 . 1} \\
102.4\end{array}$ & $\begin{array}{l}57120 \\
10752\end{array}$ \\
\hline 302 & $\begin{array}{c}\text { Sun } \\
\text { HPC } 10000400 \mathrm{MHz} \text { Cluster }\end{array}$ & $\begin{array}{c}\text { Consulting } \\
\text { London UK / } 2000\end{array}$ & Industry & 128 & $\begin{array}{r}\mathbf{8 0 . 1} \\
102.4\end{array}$ & $\begin{array}{l}57120 \\
10752\end{array}$ \\
\hline 303 & $\begin{array}{c}\text { Sun } \\
\text { HPC } 10000400 \mathrm{MHz} \text { Cluster }\end{array}$ & $\begin{array}{l}\text { Convergys Corporation } \\
\text { Lake Mary USA / } 2000\end{array}$ & $\begin{array}{l}\text { Industry } \\
\text { Finance }\end{array}$ & 128 & $\begin{array}{l}\mathbf{8 0 . 1} \\
102.4\end{array}$ & $\begin{array}{l}57120 \\
10752\end{array}$ \\
\hline 304 & $\begin{array}{c}\text { Sun } \\
\text { HPC } 10000400 \mathrm{MHz} \text { Cluster }\end{array}$ & $\begin{array}{c}\text { E-commerce } \\
\text { Chiyoda-ku Japan /2000 }\end{array}$ & $\begin{array}{l}\text { Industry } \\
\text { WWW }\end{array}$ & 128 & $\begin{array}{r}\mathbf{8 0 . 1} \\
102.4\end{array}$ & $\begin{array}{l}57120 \\
10752\end{array}$ \\
\hline 305 & $\begin{array}{c}\text { Sun } \\
\text { HPC } 10000400 \mathrm{MHz} \text { Cluster }\end{array}$ & $\begin{array}{l}\text { Electronics } \\
\text { USA / } 2000\end{array}$ & Industry & 128 & $\begin{array}{r}\mathbf{8 0 . 1} \\
102.4\end{array}$ & $\begin{array}{l}57120 \\
10752\end{array}$ \\
\hline 306 & $\begin{array}{c}\text { Sun } \\
\text { HPC } 10000400 \mathrm{MHz} \text { Cluster }\end{array}$ & $\begin{array}{l}\text { Ford Motor Company } \\
\text { Brentwood UK / } 2000\end{array}$ & $\begin{array}{l}\text { Industry } \\
\text { Automotive }\end{array}$ & 128 & $\begin{array}{l}\mathbf{8 0 . 1} \\
102.4\end{array}$ & $\begin{array}{l}57120 \\
10752\end{array}$ \\
\hline 307 & $\begin{array}{c}\text { Sun } \\
\text { HPC } 10000400 \mathrm{MHz} \text { Cluster }\end{array}$ & $\begin{array}{c}\text { Internet } \\
\text { Kyushu Japan /2000 }\end{array}$ & $\begin{array}{l}\text { Industry } \\
\text { WWW }\end{array}$ & 128 & $\begin{array}{l}\mathbf{8 0 . 1} \\
102.4\end{array}$ & $\begin{array}{l}57120 \\
10752\end{array}$ \\
\hline 308 & $\begin{array}{c}\text { Sun } \\
\text { HPC } 10000400 \mathrm{MHz} \text { Cluster }\end{array}$ & $\begin{array}{c}\text { Internet } \\
\text { Osaka Japan } / 2000\end{array}$ & $\begin{array}{l}\text { Industry } \\
\text { WWW }\end{array}$ & 128 & $\begin{array}{l}\mathbf{8 0 . 1} \\
102.4\end{array}$ & $\begin{array}{l}57120 \\
10752\end{array}$ \\
\hline 309 & $\begin{array}{c}\text { Sun } \\
\text { HPC } 10000400 \mathrm{MHz} \text { Cluster }\end{array}$ & $\begin{array}{c}\text { Jside.Com } \\
\text { Tokyo Japan } / 2000\end{array}$ & Industry & 128 & $\begin{array}{l}\mathbf{8 0 . 1} \\
102.4\end{array}$ & $\begin{array}{l}57120 \\
10752\end{array}$ \\
\hline 310 & $\begin{array}{c}\text { Sun } \\
\text { HPC } 10000400 \mathrm{MHz} \text { Cluster }\end{array}$ & $\begin{array}{c}\text { Oil Company } \\
\text { Calgary Canada /2000 }\end{array}$ & $\begin{array}{l}\text { Industry } \\
\text { Geophysics }\end{array}$ & 128 & $\begin{array}{r}\mathbf{8 0 . 1} \\
102.4\end{array}$ & $\begin{array}{l}57120 \\
10752\end{array}$ \\
\hline 311 & $\begin{array}{c}\text { Sun } \\
\text { HPC } 10000400 \mathrm{MHz} \text { Cluster }\end{array}$ & $\begin{array}{l}\text { Telecommunication } \\
\text { Milano Italy } / 2000\end{array}$ & $\begin{array}{l}\text { Industry } \\
\text { Telecomm }\end{array}$ & 128 & $\begin{array}{l}\mathbf{8 0 . 1} \\
102.4\end{array}$ & $\begin{array}{l}57120 \\
10752\end{array}$ \\
\hline 312 & $\begin{array}{c}\text { Sun } \\
\text { HPC } 10000400 \mathrm{MHz} \text { Cluster }\end{array}$ & $\begin{array}{l}\text { Telecommunication Company } \\
\text { Fort Wayne USA / } 2000\end{array}$ & $\begin{array}{l}\text { Industry } \\
\text { Telecomm }\end{array}$ & 128 & $\begin{array}{l}\mathbf{8 0 . 1} \\
102.4\end{array}$ & $\begin{array}{l}57120 \\
10752\end{array}$ \\
\hline 313 & $\begin{array}{c}\text { Sun } \\
\text { HPC } 10000400 \mathrm{MHz} \text { Cluster }\end{array}$ & $\begin{array}{c}\text { Telecommunication Company } \\
\text { Freehold USA / } 2000\end{array}$ & $\begin{array}{l}\text { Industry } \\
\text { Telecomm }\end{array}$ & 128 & $\begin{array}{r}\mathbf{8 0 . 1} \\
102.4\end{array}$ & $\begin{array}{l}57120 \\
10752\end{array}$ \\
\hline 314 & $\begin{array}{c}\text { Sun } \\
\text { HPC } 10000400 \mathrm{MHz} \text { Cluster }\end{array}$ & $\begin{array}{l}\text { Telecommunication Company } \\
\text { Little Rock USA / } 2000\end{array}$ & $\begin{array}{l}\text { Industry } \\
\text { Telecomm }\end{array}$ & 128 & $\begin{array}{r}\mathbf{8 0 . 1} \\
102.4\end{array}$ & $\begin{array}{l}57120 \\
10752\end{array}$ \\
\hline 315 & $\begin{array}{c}\text { Sun } \\
\text { HPC } 10000400 \mathrm{MHz} \text { Cluster }\end{array}$ & $\begin{array}{l}\text { Telecommunication Company } \\
\text { Sacramento USA / } 2000\end{array}$ & $\begin{array}{l}\text { Industry } \\
\text { Telecomm }\end{array}$ & 128 & $\begin{array}{r}\mathbf{8 0 . 1} \\
102.4\end{array}$ & $\begin{array}{l}57120 \\
10752\end{array}$ \\
\hline 316 & $\begin{array}{c}\text { IBM } \\
\text { SP Power3 } 375 \mathrm{MHz}\end{array}$ & $\begin{array}{c}\text { TRW } \\
\text { Cleveland USA /2000 }\end{array}$ & $\begin{array}{l}\text { Industry } \\
\text { Automotive }\end{array}$ & 76 & $\begin{array}{r}79.9 \\
114\end{array}$ & \\
\hline 317 & $\begin{array}{c}\text { IBM } \\
\text { SP Power3 } 375 \mathrm{MHz}\end{array}$ & $\begin{array}{l}\text { ThyssenKrupp Information Systems } \\
\text { Germany } / 2000\end{array}$ & $\begin{array}{l}\text { Industry } \\
\text { Mechanics }\end{array}$ & 76 & $\begin{array}{r}\mathbf{7 9 . 9} \\
114\end{array}$ & \\
\hline 318 & $\begin{array}{c}\text { IBM } \\
\text { SP PC604e } 332 \mathrm{MHz}\end{array}$ & $\begin{array}{l}\text { Deutsche Bank } \\
\text { Frankfurt Germany /1999 }\end{array}$ & $\begin{array}{l}\text { Industry } \\
\text { Finance }\end{array}$ & 188 & $\begin{array}{r}\mathbf{7 9 . 5} \\
124\end{array}$ & \\
\hline 319 & $\begin{array}{c}\text { IBM } \\
\text { SP P2SC } 160 \mathrm{MHz}\end{array}$ & $\begin{array}{l}\text { Government } \\
\text { UK /1999 }\end{array}$ & Classified & 178 & $\begin{array}{l}\mathbf{7 8 . 4} \\
113.9\end{array}$ & \\
\hline 320 & $\begin{array}{c}\text { IBM } \\
\text { SP Power3 } 375 \mathrm{MHz}\end{array}$ & $\begin{array}{l}\text { GWA Web } \\
\text { USA } / 2000\end{array}$ & Industry & 74 & $\begin{array}{r}77.9 \\
111\end{array}$ & \\
\hline
\end{tabular}

Mannheim/Tennessee November 2, 2000 
Top500 Supercomputers - Worldwide

\begin{tabular}{|c|c|c|c|c|c|c|}
\hline $\begin{array}{r}\mathbf{N} \\
\text { world }\end{array}$ & $\begin{array}{l}\text { Manufacturer } \\
\text { Computer }\end{array}$ & $\begin{array}{l}\text { Installation Site } \\
\text { Location/Year }\end{array}$ & $\begin{array}{c}\text { Field of } \\
\text { Application }\end{array}$ & $\begin{array}{c}\# \\
\text { Proc. }\end{array}$ & $\begin{array}{r}\mathbf{R}_{\max } \\
R_{\text {peak }} \\
\text { [Gflop/s] }\end{array}$ & $\begin{array}{r}N_{\max } \\
N_{1 / 2}\end{array}$ \\
\hline 321 & $\begin{array}{c}\text { IBM } \\
\text { SP Power3 } 375 \mathrm{MHz}\end{array}$ & $\begin{array}{l}\text { comdirekt Bank } \\
\text { Germany /2000 }\end{array}$ & $\begin{array}{l}\text { Industry } \\
\text { Finance }\end{array}$ & 74 & $\begin{array}{r}77.9 \\
111\end{array}$ & \\
\hline 322 & $\begin{array}{c}\text { IBM } \\
\text { SP PC604e } 332 \mathrm{MHz}\end{array}$ & $\begin{array}{c}\text { RWE } \\
\text { Germany /2000 }\end{array}$ & Industry & 184 & $\begin{array}{r}\mathbf{7 7 . 8} \\
122\end{array}$ & . \\
\hline 323 & $\begin{array}{c}\text { IBM } \\
\text { SP PC604e } 332 \mathrm{MHz}\end{array}$ & $\begin{array}{c}\text { TELUS Communications Inc. } \\
\text { Canada /1998 }\end{array}$ & $\begin{array}{l}\text { Industry } \\
\text { Telecomm }\end{array}$ & 184 & $\begin{array}{r}77.8 \\
122\end{array}$ & \\
\hline 324 & $\begin{array}{c}\text { NEC } \\
\mathrm{SX}-4 / 40 \mathrm{H} 2\end{array}$ & $\begin{array}{c}\text { HWW/Universitaet Stuttgart } \\
\text { Stuttgart Germany /1999 }\end{array}$ & Industry & 40 & $\begin{array}{r}77.2 \\
80\end{array}$ & . \\
\hline 325 & $\begin{array}{c}\text { Sun } \\
\text { HPC } 420450 \mathrm{MHz} \text { Cluster }\end{array}$ & $\begin{array}{c}\text { Internet Service Provider } \\
\text { USA } / 2000\end{array}$ & $\begin{array}{l}\text { Industry } \\
\text { WWW }\end{array}$ & 112 & $\begin{array}{r}\mathbf{7 7 . 0 1} \\
100.8\end{array}$ & . \\
\hline 326 & $\begin{array}{c}\text { SGI } \\
\text { ORIGIN } 2000\end{array}$ & $\begin{array}{c}\text { Naval Oceanographic Office (NAVOCEANO) } \\
\text { Bay Saint Louis USA /1999 }\end{array}$ & $\begin{array}{l}\text { Research } \\
\text { Aerospace }\end{array}$ & 256 & $\begin{array}{l}\mathbf{7 6 . 9} \\
99.84\end{array}$ & . \\
\hline 327 & $\begin{array}{c}\text { IBM } \\
\text { SP Power3 } 200 \mathrm{MHz}\end{array}$ & $\begin{array}{c}\text { Deutsche Telekom AG } \\
\text { Darmstadt Germany /1999 }\end{array}$ & $\begin{array}{l}\text { Industry } \\
\text { Telecomm }\end{array}$ & 128 & $\begin{array}{r}76.77 \\
102\end{array}$ & $\begin{array}{l}89000 \\
11500\end{array}$ \\
\hline 328 & $\begin{array}{c}\text { IBM } \\
\text { SP Power3 } 200 \mathrm{MHz}\end{array}$ & $\begin{array}{c}\text { IBM Research } \\
\text { Switzerland /1999 }\end{array}$ & Research & 128 & $\begin{array}{r}76.77 \\
102\end{array}$ & $\begin{array}{l}89000 \\
11500\end{array}$ \\
\hline 329 & $\begin{array}{c}\text { IBM } \\
\text { SP Power3 } 375 \mathrm{MHz}\end{array}$ & $\begin{array}{l}\text { British Airways } \\
\text { UK } / 2000\end{array}$ & $\begin{array}{l}\text { Industry } \\
\text { Transportation }\end{array}$ & 72 & $\begin{array}{r}75.9 \\
108\end{array}$ & \\
\hline 330 & $\begin{array}{c}\text { IBM } \\
\text { SP Power3 } 375 \mathrm{MHz}\end{array}$ & $\begin{array}{l}\text { Ensign Geophysics } \\
\text { UK } / 2000\end{array}$ & $\begin{array}{l}\text { Industry } \\
\text { Geophysics }\end{array}$ & 72 & $\begin{array}{r}75.9 \\
108\end{array}$ & \\
\hline 331 & $\begin{array}{c}\text { IBM } \\
\text { SP Power3 } 375 \mathrm{MHz}\end{array}$ & $\begin{array}{l}\text { Georgia Institute of Technology } \\
\text { Atlanta USA / } 2000\end{array}$ & Research & 72 & $\begin{array}{r}75.9 \\
108\end{array}$ & \\
\hline 332 & $\begin{array}{c}\text { Sun } \\
\text { HPC } 10000400 \mathrm{MHz} \text { Cluster }\end{array}$ & $\begin{array}{c}\text { Bank } \\
\text { Leeds UK /2000 }\end{array}$ & $\begin{array}{l}\text { Industry } \\
\text { Finance }\end{array}$ & 120 & $\begin{array}{r}75.8 \\
96\end{array}$ & \\
\hline 333 & $\begin{array}{c}\text { Sun } \\
\text { HPC } 10000400 \mathrm{MHz} \text { Cluster }\end{array}$ & $\begin{array}{c}\text { Dutchtone } \\
\text { Den Haag Netherlands /2000 }\end{array}$ & $\begin{array}{l}\text { Industry } \\
\text { Telecomm }\end{array}$ & 120 & $\begin{array}{r}75.8 \\
96\end{array}$ & · \\
\hline 334 & $\begin{array}{c}\text { Sun } \\
\text { HPC } 10000400 \mathrm{MHz} \text { Cluster }\end{array}$ & $\begin{array}{c}\text { T-Online } \\
\text { Weiterstadt Germany } / 2000\end{array}$ & $\begin{array}{l}\text { Industry } \\
\text { Telecomm }\end{array}$ & 120 & $\begin{array}{r}75.8 \\
96\end{array}$ & \\
\hline 335 & $\begin{array}{c}\text { Sun } \\
\text { HPC } 10000400 \mathrm{MHz} \text { Cluster }\end{array}$ & $\begin{array}{l}\text { Telecommunication } \\
\text { Italy } / 2000\end{array}$ & $\begin{array}{l}\text { Industry } \\
\text { Telecomm }\end{array}$ & 120 & $\begin{array}{r}75.8 \\
96\end{array}$ & \\
\hline 336 & $\begin{array}{c}\text { Sun } \\
\text { HPC } 10000400 \mathrm{MHz} \text { Cluster }\end{array}$ & $\begin{array}{l}\text { Telecommunication } \\
\text { Italy } / 2000\end{array}$ & $\begin{array}{l}\text { Industry } \\
\text { Telecomm }\end{array}$ & 120 & $\begin{array}{r}75.8 \\
96\end{array}$ & . \\
\hline 337 & $\begin{array}{c}\text { Sun } \\
\text { HPC } 10000400 \mathrm{MHz} \text { Cluster }\end{array}$ & $\begin{array}{c}\text { Media } \\
\text { Barcelona Spain } / 2000\end{array}$ & Industry & 80 & $\begin{array}{r}75.8 \\
64\end{array}$ & $\begin{array}{l}\cdot \\
.\end{array}$ \\
\hline 338 & $\begin{array}{c}\text { IBM } \\
\text { SP PC604e } 332 \mathrm{MHz}\end{array}$ & $\begin{array}{l}\text { FT UEI Antony } \\
\text { France } / 2000\end{array}$ & Industry & 178 & $\begin{array}{r}75.2 \\
118\end{array}$ & . \\
\hline 339 & $\begin{array}{c}\text { Sun } \\
\text { HPC } 10000400 \mathrm{MHz} \text { Cluster }\end{array}$ & $\begin{array}{c}\text { debis Systemhaus } \\
\text { Stuttgart Germany /1999 }\end{array}$ & $\begin{array}{l}\text { Industry } \\
\text { Automotive }\end{array}$ & 120 & $\begin{array}{r}74.89 \\
96\end{array}$ & . \\
\hline 340 & $\begin{array}{l}\text { Cray Inc. } \\
\text { T3E }\end{array}$ & $\begin{array}{l}\text { NRI for Earth Science and Disaster (NIED) } \\
\text { Japan / } 1997\end{array}$ & Research & 172 & $\begin{array}{l}\mathbf{7 4 . 5} \\
103.2\end{array}$ & . \\
\hline
\end{tabular}

Mannheim/Tennessee November 2, 2000 
TOP500 Supercomputers - Worldwide

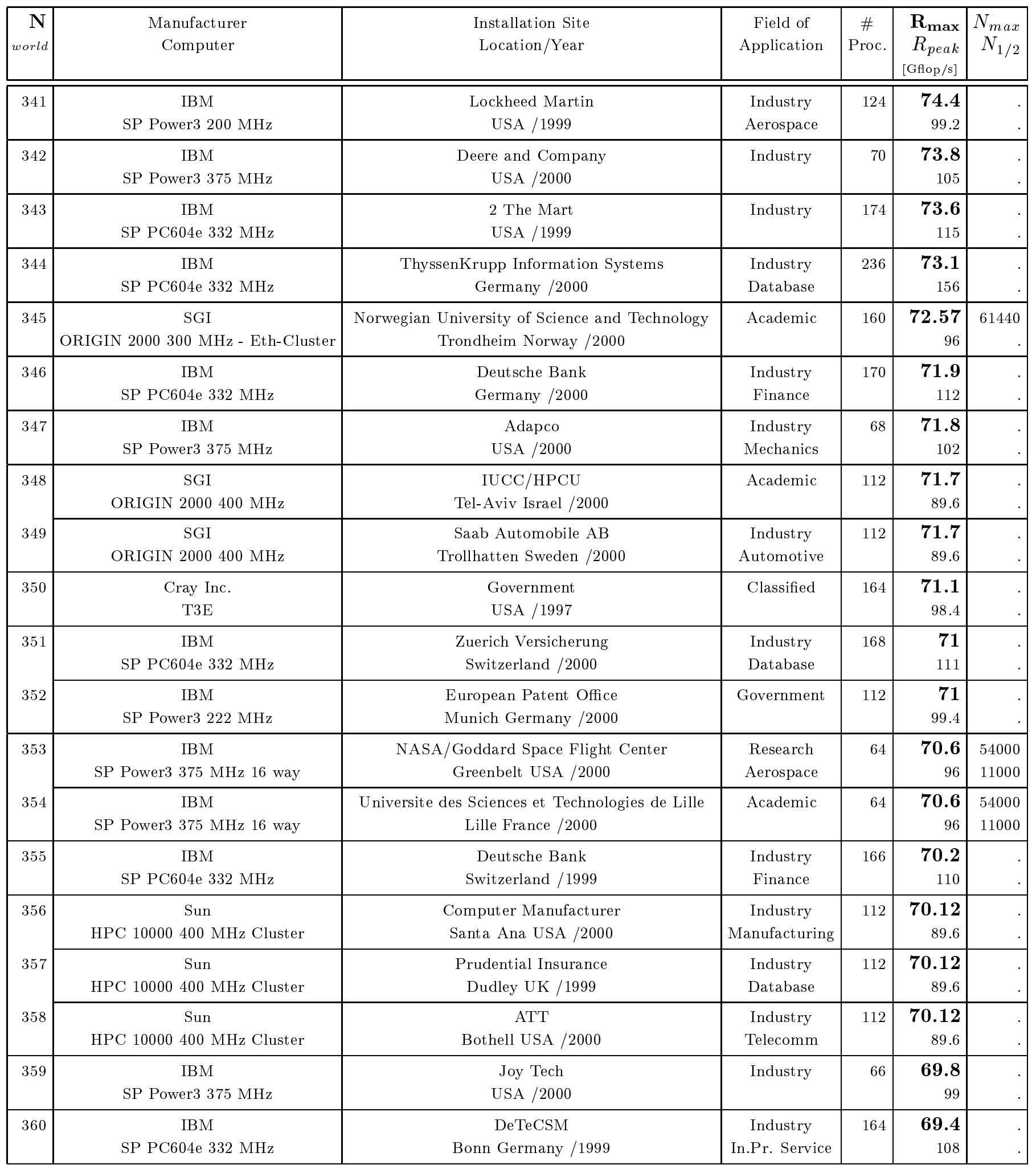


TOP500 Supercomputers - Worldwide

\begin{tabular}{|c|c|c|c|c|c|c|}
\hline $\begin{array}{r}\mathbf{N} \\
\text { world }\end{array}$ & $\begin{array}{l}\text { Manufacturer } \\
\text { Computer }\end{array}$ & $\begin{array}{l}\text { Installation Site } \\
\text { Location/Year }\end{array}$ & $\begin{array}{c}\text { Field of } \\
\text { Application }\end{array}$ & $\begin{array}{c}\# \\
\text { Proc. }\end{array}$ & $\begin{array}{r}\mathbf{R}_{\max } \\
R_{\text {peak }} \\
\text { [Gflop/s] }\end{array}$ & $\begin{array}{r}N_{\max } \\
N_{1 / 2}\end{array}$ \\
\hline 361 & $\begin{array}{c}\mathrm{NEC} \\
\mathrm{SX}-4 / 36 \mathrm{H} 2\end{array}$ & $\begin{array}{c}\text { National Institute for Environmental Studies } \\
\text { Tsukuba Japan /1997 }\end{array}$ & $\begin{array}{c}\text { Research } \\
\text { Environment }\end{array}$ & 36 & $\begin{array}{r}69.4 \\
72\end{array}$ & \\
\hline 362 & $\begin{array}{c}\text { IBM } \\
\text { SP2 } / 402\end{array}$ & $\begin{array}{l}\text { Chip Manufacturer (B) } \\
\text { USA /1997 }\end{array}$ & $\begin{array}{l}\text { Industry } \\
\text { Electronics }\end{array}$ & 402 & $\begin{array}{r}69.33 \\
106.53\end{array}$ & \\
\hline 363 & $\begin{array}{c}\text { Sun } \\
\text { HPC } 10000400 \mathrm{MHz} \text { Cluster }\end{array}$ & $\begin{array}{c}\text { KT Freetel } \\
\text { Seoul Korea /1999 }\end{array}$ & $\begin{array}{l}\text { Industry } \\
\text { Telecomm }\end{array}$ & 110 & $\begin{array}{r}68.77 \\
88\end{array}$ & \\
\hline 364 & $\begin{array}{c}\text { IBM } \\
\text { SP PC604e } 332 \mathrm{MHz}\end{array}$ & $\begin{array}{c}\text { BASF } \\
\text { Ludwigshafen Germany /1999 }\end{array}$ & $\begin{array}{l}\text { Industry } \\
\text { Chemistry }\end{array}$ & 162 & $\begin{array}{r}68.5 \\
107\end{array}$ & \\
\hline 365 & $\begin{array}{c}\text { IBM } \\
\text { SP PC604e } 332 \mathrm{MHz}\end{array}$ & $\begin{array}{l}\text { Dresdner Bank } \\
\text { Germany } / 2000\end{array}$ & Industry & 162 & $\begin{array}{r}68.5 \\
107\end{array}$ & \\
\hline 366 & $\begin{array}{c}\text { IBM } \\
\text { SP P2SC } 120 \mathrm{MHz}\end{array}$ & $\begin{array}{c}\text { Centre Informatique National (CINES) } \\
\text { Montpellier France /1999 }\end{array}$ & Academic & 207 & $\begin{array}{l}\mathbf{6 7 . 8} \\
99.36\end{array}$ & \\
\hline 367 & $\begin{array}{c}\text { IBM } \\
\text { SP Power3 } 375 \mathrm{MHz}\end{array}$ & $\begin{array}{c}\text { C4 / Centre Europeo del Parallelismo de Barcelona } \\
\text { Barcelona Spain } / 2000\end{array}$ & Academic & 64 & $\begin{array}{r}67.78 \\
96\end{array}$ & $\begin{array}{l}76000 \\
10400\end{array}$ \\
\hline 368 & $\begin{array}{c}\text { IBM } \\
\text { SP Power3 } 375 \mathrm{MHz}\end{array}$ & $\begin{array}{c}\text { CINECA } \\
\text { Bologna Italy } / 2000\end{array}$ & Academic & 64 & $\begin{array}{r}67.78 \\
96\end{array}$ & $\begin{array}{l}76000 \\
10400\end{array}$ \\
\hline 369 & $\begin{array}{c}\text { IBM } \\
\text { SP Power3 } 375 \mathrm{MHz}\end{array}$ & $\begin{array}{r}\text { Caltech } \\
\text { Pasadena USA /2000 }\end{array}$ & Academic & 64 & $\begin{array}{r}67.78 \\
96\end{array}$ & $\begin{array}{l}76000 \\
10400\end{array}$ \\
\hline 370 & $\begin{array}{c}\text { IBM } \\
\text { SP Power3 } 375 \mathrm{MHz}\end{array}$ & $\begin{array}{l}\text { Dassault Aviation } \\
\text { France } / 2000\end{array}$ & $\begin{array}{l}\text { Industry } \\
\text { Aerospace }\end{array}$ & 64 & $\begin{array}{r}67.78 \\
96\end{array}$ & $\begin{array}{l}76000 \\
10400\end{array}$ \\
\hline 371 & $\begin{array}{c}\text { IBM } \\
\text { SP Power3 } 375 \mathrm{MHz}\end{array}$ & $\begin{array}{l}\text { Ensign Geophysics } \\
\text { UK / } 2000\end{array}$ & $\begin{array}{l}\text { Industry } \\
\text { Geophysics }\end{array}$ & 64 & $\begin{array}{r}67.78 \\
96\end{array}$ & $\begin{array}{l}76000 \\
10400\end{array}$ \\
\hline 372 & $\begin{array}{c}\text { IBM } \\
\text { SP Power3 } 375 \mathrm{MHz}\end{array}$ & $\begin{array}{c}\text { IBM } \\
\text { Poughkeepsie USA /2000 }\end{array}$ & Vendor & 64 & $\begin{array}{r}67.78 \\
96\end{array}$ & $\begin{array}{l}76000 \\
10400\end{array}$ \\
\hline 373 & $\begin{array}{c}\text { IBM } \\
\text { SP Power3 } 375 \mathrm{MHz}\end{array}$ & $\begin{array}{c}\text { Indiana University } \\
\text { USA } / 2000\end{array}$ & Academic & 64 & $\begin{array}{r}67.78 \\
96\end{array}$ & $\begin{array}{l}76000 \\
10400\end{array}$ \\
\hline 374 & $\begin{array}{c}\text { IBM } \\
\text { SP Power3 } 375 \mathrm{MHz}\end{array}$ & $\begin{array}{c}\text { Manufacturing Company } \\
\text { Japan } / 2000\end{array}$ & $\begin{array}{c}\text { Industry } \\
\text { Manufacturing }\end{array}$ & 64 & $\begin{array}{r}67.78 \\
96\end{array}$ & $\begin{array}{l}76000 \\
10400\end{array}$ \\
\hline 375 & $\begin{array}{c}\text { IBM } \\
\text { SP Power3 } 375 \mathrm{MHz}\end{array}$ & $\begin{array}{c}\text { Manufacturing Company } \\
\text { Japan /2000 }\end{array}$ & \begin{tabular}{c|} 
Industry \\
Manufacturing
\end{tabular} & 64 & $\begin{array}{r}67.78 \\
96\end{array}$ & $\begin{array}{l}76000 \\
10400\end{array}$ \\
\hline 376 & $\begin{array}{c}\text { IBM } \\
\text { SP Power3 } 375 \mathrm{MHz}\end{array}$ & $\begin{array}{c}\text { National Cancer Institute } \\
\text { USA } / 2000\end{array}$ & Research & 64 & $\begin{array}{r}67.78 \\
96\end{array}$ & $\begin{array}{l}76000 \\
10400\end{array}$ \\
\hline 377 & $\begin{array}{c}\text { IBM } \\
\text { SP Power3 } 375 \mathrm{MHz}\end{array}$ & $\begin{array}{l}\text { Norwest Corp. } \\
\text { USA / } 2000\end{array}$ & $\begin{array}{l}\text { Industry } \\
\text { Finance }\end{array}$ & 64 & $\begin{array}{r}67.78 \\
96\end{array}$ & $\begin{array}{l}76000 \\
10400\end{array}$ \\
\hline 378 & $\begin{array}{c}\text { IBM } \\
\text { SP Power3 } 375 \mathrm{MHz}\end{array}$ & $\begin{array}{c}\text { PSA Peugeot Citroen } \\
\text { France } / 2000\end{array}$ & $\begin{array}{l}\text { Industry } \\
\text { Automotive }\end{array}$ & 64 & $\begin{array}{r}67.78 \\
96\end{array}$ & $\begin{array}{l}76000 \\
10400\end{array}$ \\
\hline 379 & $\begin{array}{c}\text { IBM } \\
\text { SP Power3 } 375 \mathrm{MHz}\end{array}$ & $\begin{array}{l}\text { Philip Morris } \\
\text { USA / } 2000\end{array}$ & Industry & 64 & $\begin{array}{r}67.78 \\
96\end{array}$ & $\begin{array}{l}76000 \\
10400\end{array}$ \\
\hline 380 & $\begin{array}{c}\text { IBM } \\
\text { SP Power3 } 375 \mathrm{MHz}\end{array}$ & $\begin{array}{c}\text { Science Applications International Corporation (SAIC) } \\
\text { USA } / 2000\end{array}$ & Industry & 64 & $\begin{array}{r}67.78 \\
96\end{array}$ & $\begin{array}{l}76000 \\
10400\end{array}$ \\
\hline
\end{tabular}

Mannheim/Tennessee November 2, 2000 
Top500 Supercomputers - Worldwide

\begin{tabular}{|c|c|c|c|c|c|c|}
\hline $\begin{array}{r}\mathbf{N} \\
\text { world }\end{array}$ & $\begin{array}{l}\text { Manufacturer } \\
\text { Computer }\end{array}$ & $\begin{array}{l}\text { Installation Site } \\
\text { Location/Year }\end{array}$ & $\begin{array}{c}\text { Field of } \\
\text { Application }\end{array}$ & $\begin{array}{c}\# \\
\text { Proc. }\end{array}$ & $\begin{array}{r}\mathbf{R}_{\text {max }} \\
R_{\text {peak }} \\
\text { [Gflop/s] }\end{array}$ & $\begin{array}{l}N_{m a} \\
N_{1}\end{array}$ \\
\hline 381 & $\begin{array}{c}\text { IBM } \\
\text { SP Power3 } 375 \mathrm{MHz}\end{array}$ & $\begin{array}{c}\text { Volvo } \\
\text { Gothenberg Sweden } / 2000\end{array}$ & $\begin{array}{l}\text { Industry } \\
\text { Automotive }\end{array}$ & 64 & $\begin{array}{r}67.78 \\
96\end{array}$ & $\begin{array}{l}7600 \\
1040\end{array}$ \\
\hline 382 & $\begin{array}{c}\text { IBM } \\
\text { SP Power3 } 375 \mathrm{MHz}\end{array}$ & $\begin{array}{l}\text { Western Geophysical } \\
\text { Houston USA / } 2000\end{array}$ & $\begin{array}{l}\text { Industry } \\
\text { Geophysics }\end{array}$ & 64 & $\begin{array}{r}67.78 \\
96\end{array}$ & $\begin{array}{l}7600 \\
1040\end{array}$ \\
\hline 383 & $\begin{array}{c}\text { IBM } \\
\text { SP PC604e } 332 \mathrm{MHz}\end{array}$ & $\begin{array}{c}\text { SOGEI } \\
\text { Italy } / 1998\end{array}$ & Government & 160 & $\begin{array}{r}67.7 \\
106\end{array}$ & \\
\hline 384 & $\begin{array}{c}\text { IBM } \\
\text { SP PC604e } 332 \mathrm{MHz}\end{array}$ & $\begin{array}{c}\text { Telecom Italia } \\
\text { Italy /1998 }\end{array}$ & $\begin{array}{l}\text { Industry } \\
\text { Telecomm }\end{array}$ & 160 & $\begin{array}{r}67.7 \\
106\end{array}$ & \\
\hline 385 & $\begin{array}{l}\text { Cray Inc. } \\
\text { T3E900 }\end{array}$ & $\begin{array}{l}\text { Government } \\
\text { USA /1998 }\end{array}$ & Classified & 108 & $\begin{array}{r}\mathbf{6 7 . 6} \\
97.2\end{array}$ & \\
\hline 386 & $\begin{array}{c}\text { Sun } \\
\text { HPC } 10000333 \mathrm{MHz} \text { Cluster }\end{array}$ & $\begin{array}{c}\text { Telecommunications } \\
\text { Kanagawa Japan /2000 }\end{array}$ & $\begin{array}{l}\text { Industry } \\
\text { Telecomm }\end{array}$ & 128 & $\begin{array}{r}66.93 \\
85.2\end{array}$ & $\begin{array}{l}5712 \\
1008\end{array}$ \\
\hline 387 & $\begin{array}{c}\text { IBM } \\
\text { SP PC604e } 332 \mathrm{MHz}\end{array}$ & $\begin{array}{c}\text { Government } \\
\text { France /1999 }\end{array}$ & Classified & 158 & $\begin{array}{r}66.8 \\
104\end{array}$ & \\
\hline 388 & $\begin{array}{c}\text { SGI } \\
\text { ORIGIN } 2000250 \mathrm{MHz} \text { - Eth-Cluster }\end{array}$ & $\begin{array}{l}\text { NASA/Ames Research Center/NAS } \\
\text { Mountain View USA / } 2000\end{array}$ & $\begin{array}{l}\text { Research } \\
\text { Aerospace }\end{array}$ & 192 & $\begin{array}{r}66.7 \\
96\end{array}$ & \\
\hline 389 & $\begin{array}{c}\text { SGI } \\
\text { ORIGIN } 2000400 \mathrm{MHz}\end{array}$ & $\begin{array}{c}\text { University of Arizona } \\
\text { Tucson USA / } 2000\end{array}$ & Academic & 104 & $\begin{array}{r}\mathbf{6 6 . 6} \\
83.2\end{array}$ & \\
\hline 390 & $\begin{array}{c}\text { IBM } \\
\text { SP PC604e } 332 \mathrm{MHz}\end{array}$ & $\begin{array}{c}\text { Federal Express } \\
\text { USA /1999 }\end{array}$ & $\begin{array}{l}\text { Industry } \\
\text { Database }\end{array}$ & 156 & $\begin{array}{r}66 \\
103\end{array}$ & \\
\hline 391 & $\begin{array}{c}\text { IBM } \\
\text { SP PC604e } 332 \mathrm{MHz}\end{array}$ & $\begin{array}{c}\text { TRW } \\
\text { Cleveland USA /1999 }\end{array}$ & $\begin{array}{l}\text { Industry } \\
\text { Automotive }\end{array}$ & 156 & $\begin{array}{r}66 \\
103\end{array}$ & \\
\hline 392 & $\begin{array}{c}\text { Sun } \\
\text { HPC } 420450 \mathrm{MHz} \text { Cluster }\end{array}$ & $\begin{array}{c}\text { Monsanto } \\
\text { Cambridge USA /2000 }\end{array}$ & $\begin{array}{l}\text { Industry } \\
\text { Biology }\end{array}$ & 96 & $\begin{array}{r}65.8 \\
86.4\end{array}$ & \\
\hline 393 & $\begin{array}{c}\text { Sun } \\
\text { HPC } 450400 \mathrm{MHz} \text { Cluster }\end{array}$ & $\begin{array}{l}\text { Monsanto } \\
\text { St. Loius USA /2000 }\end{array}$ & $\begin{array}{l}\text { Industry } \\
\text { Biology }\end{array}$ & 96 & $\begin{array}{r}65.8 \\
76.8\end{array}$ & \\
\hline 394 & $\begin{array}{c}\text { IBM } \\
\text { SP P2SC } 160 \mathrm{MHz}\end{array}$ & $\begin{array}{l}\text { KTH - Royal Institute of Technology } \\
\text { Stockholm Sweden /1998 }\end{array}$ & Research & 146 & $\begin{array}{l}64.8 \\
93.44\end{array}$ & \\
\hline 395 & $\begin{array}{c}\text { Sun } \\
\text { HPC } 10000400 \mathrm{MHz} \text { Cluster }\end{array}$ & $\begin{array}{c}\text { Telecommunication } \\
\text { Rio de Janeiro Brazil /2000 }\end{array}$ & $\begin{array}{l}\text { Industry } \\
\text { Telecomm }\end{array}$ & 104 & $\begin{array}{r}64.73 \\
83.2\end{array}$ & \\
\hline 396 & $\begin{array}{c}\text { Self-made } \\
\text { SCore II/PIII } 800 \mathrm{MHz}\end{array}$ & $\begin{array}{c}\text { Real World Computing (RWCP)/Tsukuba Research Center } \\
\text { Tsukuba-shi Japan } / 2000\end{array}$ & Research & 132 & $\begin{array}{r}64.7 \\
105\end{array}$ & $\begin{array}{r}5800 \\
800\end{array}$ \\
\hline 397 & $\begin{array}{c}\text { SGI } \\
\text { ORIGIN } 3000400 \mathrm{MHz}\end{array}$ & $\begin{array}{c}\text { Centre Informatique National (CINES) } \\
\text { Montpellier France } / 2000\end{array}$ & Research & 96 & $\begin{array}{r}64.2 \\
76.8\end{array}$ & \\
\hline 398 & $\begin{array}{c}\text { Hewlett-Packard } \\
\text { N4000 } 440 \mathrm{MHz} / \text { HyperPlex }\end{array}$ & $\begin{array}{l}\text { DaimlerChrysler } \\
\text { USA / } 2000\end{array}$ & $\begin{array}{l}\text { Industry } \\
\text { Automotive }\end{array}$ & 96 & $\begin{array}{r}\mathbf{6 3 . 8} \\
168.96\end{array}$ & \\
\hline 399 & $\begin{array}{c}\text { Hewlett-Packard } \\
\text { N4000 } 440 \mathrm{MHz} / \text { HyperPlex }\end{array}$ & $\begin{array}{l}\text { University of Kentucky } \\
\text { Lexington USA / } 2000\end{array}$ & Academic & 96 & $\begin{array}{r}\mathbf{6 3 . 8} \\
168.96\end{array}$ & \\
\hline 400 & $\begin{array}{c}\text { Hewlett-Packard } \\
\text { N4000 } 440 \mathrm{MHz} / \text { HyperPlex }\end{array}$ & $\begin{array}{c}\text { VW (Volkswagen AG) } \\
\text { Wolfsburg Germany /2000 }\end{array}$ & $\begin{array}{l}\text { Industry } \\
\text { Automotive }\end{array}$ & 96 & $\begin{array}{r}\mathbf{6 3 . 8} \\
168.96\end{array}$ & \\
\hline
\end{tabular}


Top500 Supercomputers - Worldwide

\begin{tabular}{|c|c|c|c|c|c|c|}
\hline $\begin{array}{r}\mathbf{N} \\
\text { world }\end{array}$ & $\begin{array}{l}\text { Manufacturer } \\
\text { Computer }\end{array}$ & $\begin{array}{l}\text { Installation Site } \\
\text { Location/Year }\end{array}$ & $\begin{array}{c}\text { Field of } \\
\text { Application }\end{array}$ & $\begin{array}{c}\# \\
\text { Proc. }\end{array}$ & $\begin{array}{r}\mathbf{R}_{\max } \\
R_{\text {peak }} \\
\text { [Gflop/s] } \\
\end{array}$ & $\begin{array}{r}N_{\max } \\
N_{1 / 2}\end{array}$ \\
\hline 401 & $\begin{array}{c}\text { Compaq } \\
\text { AlphaServer SC ES40/EV67 }\end{array}$ & $\begin{array}{l}\text { Los Alamos National Laboratory } \\
\text { Los Alamos USA / } 2000\end{array}$ & Research & 64 & $\begin{array}{r}63.8 \\
85.4\end{array}$ & $\begin{array}{r}53000 \\
9000\end{array}$ \\
\hline 402 & $\begin{array}{c}\text { Compaq } \\
\text { AlphaServer SC ES40/EV67 }\end{array}$ & $\begin{array}{c}\text { Oak Ridge National Laboratory } \\
\text { Oak Ridge USA / } 2000\end{array}$ & Research & 64 & $\begin{array}{r}\mathbf{6 3 . 8} \\
85.4\end{array}$ & $\begin{array}{r}53000 \\
9000\end{array}$ \\
\hline 403 & $\begin{array}{c}\text { Compaq } \\
\text { AlphaServer SC ES40/EV67 }\end{array}$ & $\begin{array}{c}\text { Wright-Patterson Air Force Base/DoD ASC } \\
\text { USA } / 2000\end{array}$ & Research & 64 & $\begin{array}{r}63.8 \\
85.4\end{array}$ & $\begin{array}{r}53000 \\
9000\end{array}$ \\
\hline 404 & $\begin{array}{c}\text { IBM } \\
\text { SP Power3 } 375 \mathrm{MHz}\end{array}$ & $\begin{array}{l}\text { Canadian National Defense } \\
\text { Canada } / 2000\end{array}$ & Classified & 60 & $\begin{array}{r}63.6 \\
90\end{array}$ & \\
\hline 405 & $\begin{array}{c}\text { IBM } \\
\text { SP Power3 } 375 \mathrm{MHz}\end{array}$ & $\begin{array}{c}\text { Osaka University - Research Center for Nuclear Physics } \\
\text { Osaka Japan } / 2000\end{array}$ & Academic & 60 & $\begin{array}{r}63.6 \\
90\end{array}$ & . \\
\hline 406 & $\begin{array}{c}\text { IBM } \\
\text { SP Power3 } 375 \mathrm{MHz}\end{array}$ & $\begin{array}{l}\text { Pioneer-Standard Electronics } \\
\text { USA / } 2000\end{array}$ & Industry & 60 & $\begin{array}{r}63.6 \\
90\end{array}$ & . \\
\hline 407 & $\begin{array}{c}\text { IBM } \\
\text { SP Power3 } 375 \mathrm{MHz}\end{array}$ & $\begin{array}{l}\text { Soccer Lottery } \\
\text { Japan } / 2000\end{array}$ & Government & 60 & $\begin{array}{r}63.6 \\
90\end{array}$ & . \\
\hline 408 & $\begin{array}{c}\text { SGI } \\
\text { ORIGIN } 2000\end{array}$ & $\begin{array}{c}\text { Sandia National Labs } \\
\text { Albuquerque USA /1997 }\end{array}$ & Research & 208 & $\begin{array}{l}\mathbf{6 3 . 1} \\
81.12\end{array}$ & \\
\hline 409 & $\begin{array}{c}\text { IBM } \\
\text { SP Power3 } 200 \mathrm{MHz}\end{array}$ & $\begin{array}{c}\text { Deutsche Telekom AG } \\
\text { Darmstadt Germany /1999 }\end{array}$ & $\begin{array}{l}\text { Industry } \\
\text { Telecomm }\end{array}$ & 104 & $\begin{array}{r}62.9 \\
82.9\end{array}$ & \\
\hline 410 & $\begin{array}{c}\text { IBM } \\
\text { SP PC604e } 332 \mathrm{MHz}\end{array}$ & $\begin{array}{c}\text { Axone } \\
\text { France } / 2000\end{array}$ & Industry & 148 & $\begin{array}{r}\mathbf{6 2 . 6} \\
98.2\end{array}$ & . \\
\hline 411 & $\begin{array}{c}\text { IBM } \\
\text { SP PC604e } 332 \mathrm{MHz}\end{array}$ & $\begin{array}{c}\text { Bank of America } \\
\text { USA / } 1999\end{array}$ & $\begin{array}{l}\text { Industry } \\
\text { Finance }\end{array}$ & 148 & $\begin{array}{r}\mathbf{6 2 . 6} \\
98.2\end{array}$ & . \\
\hline 412 & $\begin{array}{c}\text { IBM } \\
\text { SP PC604e } 332 \mathrm{MHz}\end{array}$ & $\begin{array}{l}\text { Sony Data } \\
\text { UK /1999 }\end{array}$ & Industry & 148 & $\begin{array}{r}\mathbf{6 2 . 6} \\
98.2\end{array}$ & . \\
\hline 413 & $\begin{array}{c}\text { Self-made } \\
\text { NT Supercluster }\end{array}$ & $\begin{array}{c}\text { NCSA } \\
\text { Urbana-Champaign USA /1999 }\end{array}$ & Research & 256 & $\begin{array}{r}\mathbf{6 2 . 5 9} \\
140.8\end{array}$ & $\begin{array}{r}122500 \\
20500\end{array}$ \\
\hline 414 & $\begin{array}{c}\text { Sun } \\
\text { HPC } 4500400 \mathrm{MHz} \text { Cluster }\end{array}$ & $\begin{array}{c}\text { Internet } \\
\text { USA / } 2000\end{array}$ & $\begin{array}{l}\text { Industry } \\
\text { WWW }\end{array}$ & 110 & $\begin{array}{r}62.27 \\
88\end{array}$ & \\
\hline 415 & $\begin{array}{c}\text { SGI } \\
\text { ORIGIN } 2000300 \mathrm{MHz}\end{array}$ & $\begin{array}{c}\text { Boeing Company } \\
\text { Huntsville USA /1999 }\end{array}$ & $\begin{array}{l}\text { Industry } \\
\text { Aerospace }\end{array}$ & 128 & $\begin{array}{r}62.25 \\
76.8\end{array}$ & $\begin{array}{r}60032 \\
9000\end{array}$ \\
\hline 416 & $\begin{array}{c}\text { SGI } \\
\text { ORIGIN } 2000300 \mathrm{MHz}\end{array}$ & $\begin{array}{c}\text { CSC (Centre for Sientific Computing) } \\
\text { Espoo Finland /1999 }\end{array}$ & Academic & 128 & $\begin{array}{r}62.25 \\
76.8\end{array}$ & $\begin{array}{r}60032 \\
9000\end{array}$ \\
\hline 417 & $\begin{array}{c}\text { SGI } \\
\text { ORIGIN } 2000300 \mathrm{MHz}\end{array}$ & $\begin{array}{c}\text { DaimlerChrysler } \\
\text { Detroit USA /1999 }\end{array}$ & $\begin{array}{l}\text { Industry } \\
\text { Automotive }\end{array}$ & 128 & $\begin{array}{r}62.25 \\
76.8\end{array}$ & $\begin{array}{r}60032 \\
9000\end{array}$ \\
\hline 418 & $\begin{array}{c}\text { SGI } \\
\text { ORIGIN } 2000300 \mathrm{MHz}\end{array}$ & $\begin{array}{c}\text { DaimlerChrysler } \\
\text { Detroit USA /1999 }\end{array}$ & $\begin{array}{l}\text { Industry } \\
\text { Automotive }\end{array}$ & 128 & $\begin{array}{r}62.25 \\
76.8\end{array}$ & $\begin{array}{r}60032 \\
9000\end{array}$ \\
\hline 419 & $\begin{array}{c}\text { SGI } \\
\text { ORIGIN } 2000300 \mathrm{MHz}\end{array}$ & $\begin{array}{c}\text { Fleet Numerical Meteorology and Oceanography Center } \\
\text { Monterey USA / } 1999\end{array}$ & $\begin{array}{l}\text { Research } \\
\text { Weather }\end{array}$ & 128 & $\begin{array}{r}62.25 \\
76.8\end{array}$ & $\begin{array}{r}60032 \\
9000\end{array}$ \\
\hline 420 & $\begin{array}{c}\text { SGI } \\
\text { ORIGIN } 2000300 \mathrm{MHz}\end{array}$ & $\begin{array}{c}\text { Ford Motor Company } \\
\text { USA / } 1999\end{array}$ & $\begin{array}{l}\text { Industry } \\
\text { Automotive }\end{array}$ & 128 & $\begin{array}{r}\mathbf{6 2 . 2 5} \\
76.8\end{array}$ & $\begin{array}{r}60032 \\
9000\end{array}$ \\
\hline
\end{tabular}

Mannheim/Tennessee November 2, 2000 
TOP500 Supercomputers - Worldwide

\begin{tabular}{|c|c|c|c|c|c|c|}
\hline $\begin{array}{r}\mathbf{N} \\
\text { world }\end{array}$ & $\begin{array}{l}\text { Manufacturer } \\
\text { Computer }\end{array}$ & $\begin{array}{l}\text { Installation Site } \\
\text { Location/Year }\end{array}$ & $\begin{array}{c}\text { Field of } \\
\text { Application }\end{array}$ & $\begin{array}{c}\# \\
\text { Proc. }\end{array}$ & $\begin{array}{r}\mathbf{R}_{\text {max }} \\
R_{\text {peak }} \\
{[\text { Gflop/s] }}\end{array}$ & $\begin{array}{r}N_{\max } \\
N_{1 / 2}\end{array}$ \\
\hline 421 & $\begin{array}{c}\text { SGI } \\
\text { ORIGIN } 2000300 \mathrm{MHz}\end{array}$ & $\begin{array}{c}\text { NASA/JPL } \\
\text { Pasadena USA /1999 }\end{array}$ & Research & 128 & $\begin{array}{r}62.25 \\
76.8\end{array}$ & $\begin{array}{r}60032 \\
9000\end{array}$ \\
\hline 422 & $\begin{array}{c}\text { SGI } \\
\text { ORIGIN } 2000300 \mathrm{MHz}\end{array}$ & $\begin{array}{l}\text { Princeton University } \\
\text { Princeton USA / } 1999\end{array}$ & Academic & 128 & $\begin{array}{r}62.25 \\
76.8\end{array}$ & $\begin{array}{r}60032 \\
9000\end{array}$ \\
\hline 423 & $\begin{array}{c}\text { SGI } \\
\text { ORIGIN } 2000300 \mathrm{MHz}\end{array}$ & $\begin{array}{c}\text { Tohoku University, Institute of Fluid Science } \\
\text { Aramaki Japan /1999 }\end{array}$ & Academic & 128 & $\begin{array}{r}62.25 \\
76.8\end{array}$ & $\begin{array}{r}60032 \\
9000\end{array}$ \\
\hline 424 & $\begin{array}{c}\text { SGI } \\
\text { ORIGIN } 2000300 \mathrm{MHz}\end{array}$ & $\begin{array}{c}\text { US Army Research Laboratory (ARL) } \\
\text { Aberdeen USA /1999 }\end{array}$ & Research & 128 & $\begin{array}{r}62.25 \\
76.8\end{array}$ & $\begin{array}{r}60032 \\
9000\end{array}$ \\
\hline 425 & $\begin{array}{c}\text { SGI } \\
\text { ORIGIN } 2000300 \mathrm{MHz}\end{array}$ & $\begin{array}{l}\text { US Army Research Laboratory (ARL) } \\
\text { Aberdeen USA /1999 }\end{array}$ & Research & 128 & $\begin{array}{r}62.25 \\
76.8\end{array}$ & $\begin{array}{r}60032 \\
9000\end{array}$ \\
\hline 426 & $\begin{array}{c}\text { SGI } \\
\text { ORIGIN } 2000300 \mathrm{MHz}\end{array}$ & $\begin{array}{c}\text { University of Tokyo/Institute for Solid State Physics } \\
\text { Tokyo Japan /1999 }\end{array}$ & Academic & 128 & $\begin{array}{r}62.25 \\
76.8\end{array}$ & $\begin{array}{r}60032 \\
9000\end{array}$ \\
\hline 427 & $\begin{array}{c}\text { SGI } \\
\text { ORIGIN } 2000300 \mathrm{MHz}\end{array}$ & $\begin{array}{c}\text { University of Tokyo/Institute for Solid State Physics } \\
\text { Tokyo Japan /1999 }\end{array}$ & Academic & 128 & $\begin{array}{r}62.25 \\
76.8\end{array}$ & $\begin{array}{r}60032 \\
9000\end{array}$ \\
\hline 428 & $\begin{array}{c}\text { SGI } \\
\text { ORIGIN } 2000300 \mathrm{MHz}\end{array}$ & $\begin{array}{c}\text { University of Tokyo/Institute for Solid State Physics } \\
\text { Tokyo Japan /1999 }\end{array}$ & Academic & 128 & $\begin{array}{r}62.25 \\
76.8\end{array}$ & $\begin{array}{r}60032 \\
9000\end{array}$ \\
\hline 429 & $\begin{array}{l}\text { Hewlett-Packard } \\
\text { V2600/HyperPlex }\end{array}$ & $\begin{array}{l}\text { Amazon.com } \\
\text { USA / } 2000\end{array}$ & $\begin{array}{l}\text { Industry } \\
\text { WWW }\end{array}$ & 64 & $\begin{array}{r}\mathbf{6 2} \\
141.31\end{array}$ & \\
\hline 430 & $\begin{array}{c}\text { IBM } \\
\text { SP Power3 } 375 \mathrm{MHz} 16 \text { way }\end{array}$ & $\begin{array}{c}\text { Ahold } \\
\text { USA } / 2000\end{array}$ & Industry & 56 & $\begin{array}{r}62 \\
84\end{array}$ & . \\
\hline 431 & $\begin{array}{c}\text { IBM } \\
\text { SP PC604e } 332 \mathrm{MHz}\end{array}$ & $\begin{array}{c}\text { Atraxis AG } \\
\text { Switzerland } / 2000\end{array}$ & $\begin{array}{c}\text { Industry } \\
\text { Transportation }\end{array}$ & 146 & $\begin{array}{r}61.8 \\
96.9\end{array}$ & . \\
\hline 432 & $\begin{array}{c}\text { NEC } \\
\text { SX-4/32 }\end{array}$ & $\begin{array}{l}\text { NEC Fuchu Plant } \\
\text { Tokyo Japan /1995 }\end{array}$ & $\begin{array}{c}\text { Vendor } \\
\text { Benchmarking }\end{array}$ & 32 & $\begin{array}{r}61.7 \\
64\end{array}$ & $\begin{array}{r}20480 \\
1688\end{array}$ \\
\hline 433 & $\begin{array}{c}\text { IBM } \\
\text { SP Power3 } 375 \mathrm{MHz}\end{array}$ & $\begin{array}{l}\text { Pitney Bowes } \\
\text { USA / } 2000\end{array}$ & Industry & 58 & $\begin{array}{r}61.5 \\
87\end{array}$ & \\
\hline 434 & $\begin{array}{l}\text { Compaq } \\
\text { Alphleet Cluster }\end{array}$ & $\begin{array}{c}\text { Institute of Physical and Chemical Res. (RIKEN) } \\
\text { Wako Japan /1999 }\end{array}$ & Research & 140 & $\begin{array}{r}61.3 \\
140\end{array}$ & $\begin{array}{l}56000 \\
22000\end{array}$ \\
\hline 435 & $\begin{array}{c}\text { IBM } \\
\text { SP PC604e } 332 \mathrm{MHz}\end{array}$ & $\begin{array}{c}\text { APAC Hong Kong (EHU) } \\
\text { Netherlands /1999 }\end{array}$ & Industry & 144 & $\begin{array}{r}60.9 \\
95.6\end{array}$ & \\
\hline 436 & $\begin{array}{c}\text { IBM } \\
\text { SP PC604e } 332 \mathrm{MHz}\end{array}$ & $\begin{array}{c}\text { EVE Bank } \\
\text { Switzerland /1999 }\end{array}$ & $\begin{array}{l}\text { Industry } \\
\text { Finance }\end{array}$ & 144 & $\begin{array}{r}60.9 \\
95.6\end{array}$ & \\
\hline 437 & $\begin{array}{c}\text { IBM } \\
\text { SP PC604e } 332 \mathrm{MHz}\end{array}$ & $\begin{array}{c}\text { France Telecom } \\
\text { France } / 2000\end{array}$ & $\begin{array}{l}\text { Industry } \\
\text { Telecomm }\end{array}$ & 144 & $\begin{array}{r}60.9 \\
95.6\end{array}$ & . \\
\hline 438 & $\begin{array}{c}\text { IBM } \\
\text { SP PC604e } 332 \mathrm{MHz}\end{array}$ & $\begin{array}{c}\text { Merck } \\
\text { Germany /1999 }\end{array}$ & $\begin{array}{c}\text { Industry } \\
\text { Pharmaceutics }\end{array}$ & 144 & $\begin{array}{r}60.9 \\
95.6\end{array}$ & \\
\hline 439 & $\begin{array}{c}\text { IBM } \\
\text { SP PC604e } 332 \mathrm{MHz}\end{array}$ & $\begin{array}{c}\text { Rabobank NED } \\
\text { USA / } 2000\end{array}$ & Industry & 144 & $\begin{array}{r}\mathbf{6 0 . 9} \\
95.6\end{array}$ & \\
\hline 440 & $\begin{array}{c}\text { IBM } \\
\text { SP PC604e } 332 \mathrm{MHz}\end{array}$ & $\begin{array}{c}\text { UBS AG } \\
\text { Switzerland /1999 }\end{array}$ & $\begin{array}{l}\text { Industry } \\
\text { Finance }\end{array}$ & 144 & $\begin{array}{r}60.9 \\
95.6\end{array}$ & \\
\hline
\end{tabular}


Top500 Supercomputers - Worldwide

\begin{tabular}{|c|c|c|c|c|c|c|}
\hline $\begin{array}{r}\mathbf{N} \\
\text { world }\end{array}$ & $\begin{array}{l}\text { Manufacturer } \\
\text { Computer }\end{array}$ & $\begin{array}{l}\text { Installation Site } \\
\text { Location/Year }\end{array}$ & $\begin{array}{c}\text { Field of } \\
\text { Application }\end{array}$ & $\begin{array}{c}\# \\
\text { Proc. }\end{array}$ & $\begin{array}{r}\mathbf{R}_{\max } \\
R_{\text {peak }} \\
\text { [Gflop/s] }\end{array}$ & $\begin{array}{r}N_{\max } \\
N_{1 / 2}\end{array}$ \\
\hline 441 & $\begin{array}{c}\text { NEC } \\
\text { SX-5/16Be }\end{array}$ & $\begin{array}{c}\text { German Aerospace Laboratory (DLR) } \\
\text { Goettingen Germany } / 2000\end{array}$ & $\begin{array}{l}\text { Research } \\
\text { Aerospace }\end{array}$ & 16 & $\begin{array}{r}60.7 \\
64\end{array}$ & \\
\hline 442 & $\begin{array}{c}\text { Sun } \\
\text { HPC } 10000400 \mathrm{MHz} \text { Cluster }\end{array}$ & $\begin{array}{l}\text { BellSouth } \\
\text { Charlotte USA / } 2000\end{array}$ & $\begin{array}{l}\text { Industry } \\
\text { Telecomm }\end{array}$ & 64 & $\begin{array}{r}\mathbf{5 9 . 7} \\
51.2\end{array}$ & \\
\hline 443 & $\begin{array}{c}\text { Sun } \\
\text { HPC } 10000400 \mathrm{MHz} \text { Cluster }\end{array}$ & $\begin{array}{c}\text { Internet } \\
\text { Yokohama Japan } / 2000\end{array}$ & $\begin{array}{l}\text { Industry } \\
\text { WWW }\end{array}$ & 64 & $\begin{array}{r}\mathbf{5 9 . 7} \\
51.2\end{array}$ & \\
\hline 444 & $\begin{array}{c}\text { Fujitsu } \\
\text { VPP500/42 }\end{array}$ & $\begin{array}{c}\text { Japan Atomic Energy Research } \\
\text { Japan /1994 }\end{array}$ & Research & 42 & $\begin{array}{r}\mathbf{5 9 . 6} \\
67.2\end{array}$ & \\
\hline 445 & $\begin{array}{c}\text { NEC } \\
\text { SX-5/8B }\end{array}$ & $\begin{array}{c}\text { National Aerospace Laboratory (NLR) } \\
\text { Noordoostpolder Netherlands /1999 }\end{array}$ & $\begin{array}{l}\text { Research } \\
\text { Aerospace }\end{array}$ & 8 & $\begin{array}{r}\mathbf{5 9 . 6} \\
64\end{array}$ & \\
\hline 446 & $\begin{array}{c}\mathrm{NEC} \\
\mathrm{SX}-5 / 8 \mathrm{~A}\end{array}$ & $\begin{array}{c}\text { Swiss Scientific Computing Center (CSCS) } \\
\text { Manno Switzerland /1999 }\end{array}$ & Research & 8 & $\begin{array}{r}\mathbf{5 9 . 6} \\
64\end{array}$ & \\
\hline 447 & $\begin{array}{c}\mathrm{NEC} \\
\mathrm{SX}-5 / 8 \mathrm{~B}\end{array}$ & $\begin{array}{c}\text { Veritas DGC } \\
\text { Crawley UK /2000 }\end{array}$ & $\begin{array}{l}\text { Industry } \\
\text { Geophysics }\end{array}$ & 8 & $\begin{array}{r}\mathbf{5 9 . 6} \\
64\end{array}$ & \\
\hline 448 & $\begin{array}{c}\text { IBM } \\
\text { SP Power3 } 375 \mathrm{MHz}\end{array}$ & $\begin{array}{c}\text { Bank of Boston } \\
\text { Pasadena USA / } 2000\end{array}$ & $\begin{array}{l}\text { Industry } \\
\text { Finance }\end{array}$ & 56 & $\begin{array}{r}\mathbf{5 9 . 4} \\
84\end{array}$ & \\
\hline 449 & $\begin{array}{c}\text { IBM } \\
\text { SP Power3 } 375 \mathrm{MHz}\end{array}$ & $\begin{array}{l}\text { Finance Company } \\
\text { Canada } / 2000\end{array}$ & $\begin{array}{l}\text { Industry } \\
\text { Finance }\end{array}$ & 56 & $\begin{array}{r}\mathbf{5 9 . 4} \\
84\end{array}$ & \\
\hline 450 & $\begin{array}{c}\text { IBM } \\
\text { SP Power3 } 375 \mathrm{MHz}\end{array}$ & $\begin{array}{c}\text { First Union National Bank } \\
\text { USA } / 2000\end{array}$ & $\begin{array}{l}\text { Industry } \\
\text { Finance }\end{array}$ & 56 & $\begin{array}{r}\mathbf{5 9 . 4} \\
84\end{array}$ & \\
\hline 451 & $\begin{array}{c}\text { IBM } \\
\text { SP Power3 } 375 \mathrm{MHz}\end{array}$ & $\begin{array}{l}\text { Frito Lay } \\
\text { USA / } 2000\end{array}$ & Industry & 56 & $\begin{array}{r}\mathbf{5 9 . 4} \\
84\end{array}$ & \\
\hline 452 & $\begin{array}{c}\text { IBM } \\
\text { SP Power3 } 375 \mathrm{MHz}\end{array}$ & $\begin{array}{c}\text { Manufacturer } \\
\text { Japan /2000 }\end{array}$ & $\begin{array}{l}\text { Industry } \\
\text { Finance }\end{array}$ & 56 & $\begin{array}{r}\mathbf{5 9 . 4} \\
84\end{array}$ & \\
\hline 453 & $\begin{array}{c}\text { IBM } \\
\text { SP Power3 } 375 \mathrm{MHz}\end{array}$ & $\begin{array}{l}\text { Prudential Securities } \\
\text { New York USA / } 2000\end{array}$ & Industry & 56 & $\begin{array}{r}\mathbf{5 9 . 4} \\
84\end{array}$ & \\
\hline 454 & $\begin{array}{c}\text { IBM } \\
\text { SP Power3 } 375 \mathrm{MHz}\end{array}$ & $\begin{array}{c}\text { Sherbrooke University } \\
\text { Sherbrooke Canada / } 2000\end{array}$ & Academic & 56 & $\begin{array}{r}\mathbf{5 9 . 4} \\
84\end{array}$ & \\
\hline 455 & $\begin{array}{c}\text { IBM } \\
\text { SP Power3 } 375 \mathrm{MHz}\end{array}$ & $\begin{array}{c}\text { United Healthcare } \\
\text { USA / } 2000\end{array}$ & $\begin{array}{l}\text { Industry } \\
\text { Database }\end{array}$ & 56 & $\begin{array}{r}\mathbf{5 9 . 4} \\
84\end{array}$ & \\
\hline 456 & $\begin{array}{c}\text { IBM } \\
\text { SP Power3 } 375 \mathrm{MHz}\end{array}$ & $\begin{array}{l}\text { West Publishing } \\
\text { USA / } 2000\end{array}$ & Industry & 56 & $\begin{array}{r}\mathbf{5 9 . 4} \\
84\end{array}$ & \\
\hline 457 & $\begin{array}{c}\text { IBM } \\
\text { SP PC604e } 332 \mathrm{MHz}\end{array}$ & $\begin{array}{c}\text { Atomic Weapons Establishment } \\
\text { Aldermaston UK /1998 }\end{array}$ & Classified & 140 & $\begin{array}{r}\mathbf{5 9 . 3} \\
92.9\end{array}$ & \\
\hline 458 & $\begin{array}{c}\text { IBM } \\
\text { SP PC604e } 332 \mathrm{MHz}\end{array}$ & $\begin{array}{c}\text { Sears } \\
\text { USA /1998 }\end{array}$ & $\begin{array}{l}\text { Industry } \\
\text { Database }\end{array}$ & 140 & $\begin{array}{r}\mathbf{5 9 . 3} \\
92.9\end{array}$ & \\
\hline 459 & $\begin{array}{c}\text { SGI } \\
\text { ORIGIN } 2000300 \mathrm{MHz}\end{array}$ & $\begin{array}{c}\text { Lunds Tekniska Hvgskola } \\
\text { Sweden /1999 }\end{array}$ & Academic & 116 & $\begin{array}{r}\mathbf{5 9 . 1} \\
69.6\end{array}$ & \\
\hline 460 & $\begin{array}{c}\text { Sun } \\
\text { HPC } 10000400 \mathrm{MHz} \text { Cluster }\end{array}$ & $\begin{array}{c}\text { Deutsche Telekom AG } \\
\text { Bamberg Germany /2000 }\end{array}$ & $\begin{array}{l}\text { Industry } \\
\text { Telecomm }\end{array}$ & 96 & $\begin{array}{r}\mathbf{5 9 . 0 4} \\
76.8\end{array}$ & \\
\hline
\end{tabular}

Mannheim/Tennessee November 2, 2000 
Top500 Supercomputers - Worldwide

\begin{tabular}{|c|c|c|c|c|c|c|}
\hline $\begin{array}{r}\mathbf{N} \\
\text { world }\end{array}$ & $\begin{array}{l}\text { Manufacturer } \\
\text { Computer }\end{array}$ & $\begin{array}{l}\text { Installation Site } \\
\text { Location/Year }\end{array}$ & $\begin{array}{c}\text { Field of } \\
\text { Application }\end{array}$ & $\begin{array}{c}\# \\
\text { Proc. }\end{array}$ & $\begin{array}{r}\mathbf{R}_{\max } \\
R_{\text {peak }} \\
{[\text { Gflop/s] }}\end{array}$ & $\begin{array}{r}N_{\max } \\
N_{1 / 2}\end{array}$ \\
\hline 461 & $\begin{array}{c}\text { Sun } \\
\text { HPC } 10000400 \mathrm{MHz} \text { Cluster }\end{array}$ & $\begin{array}{l}\text { Finance Company } \\
\text { Reston USA / } 2000\end{array}$ & $\begin{array}{l}\text { Industry } \\
\text { Finance }\end{array}$ & 96 & $\begin{array}{r}\mathbf{5 9 . 0 4} \\
76.8\end{array}$ & \\
\hline 462 & $\begin{array}{c}\text { Sun } \\
\text { HPC } 10000400 \mathrm{MHz} \text { Cluster }\end{array}$ & $\begin{array}{c}\text { NIIFD } \\
\text { Budapest Hungary /2000 }\end{array}$ & Academic & 96 & $\begin{array}{r}\mathbf{5 9 . 0 4} \\
76.8\end{array}$ & \\
\hline 463 & $\begin{array}{c}\text { Sun } \\
\text { HPC } 10000400 \mathrm{MHz} \text { Cluster }\end{array}$ & $\begin{array}{c}\text { Oil Company } \\
\text { Paris France /1999 }\end{array}$ & Industry & 96 & $\begin{array}{r}\mathbf{5 9 . 0 4} \\
76.8\end{array}$ & \\
\hline 464 & $\begin{array}{c}\text { Sun } \\
\text { HPC } 10000400 \mathrm{MHz} \text { Cluster }\end{array}$ & $\begin{array}{c}\text { Pharmaceutical } \\
\text { Stevenage UK /2000 }\end{array}$ & $\begin{array}{c}\text { Industry } \\
\text { Pharmaceutics }\end{array}$ & 96 & $\begin{array}{r}\mathbf{5 9 . 0 4} \\
76.8\end{array}$ & \\
\hline 465 & $\begin{array}{c}\text { Sun } \\
\text { HPC } 10000400 \mathrm{MHz} \text { Cluster }\end{array}$ & $\begin{array}{c}\text { Telecommunications } \\
\text { Warsaw Poland } / 2000\end{array}$ & Industry & 96 & $\begin{array}{r}\mathbf{5 9 . 0 4} \\
76.8\end{array}$ & \\
\hline 466 & $\begin{array}{c}\text { Sun } \\
\text { HPC } 10000400 \mathrm{MHz} \text { Cluster }\end{array}$ & $\begin{array}{c}\text { University of Queensland } \\
\text { Queensland Australia /2000 }\end{array}$ & Academic & 96 & $\begin{array}{r}\mathbf{5 9 . 0 4} \\
76.8\end{array}$ & \\
\hline 467 & $\begin{array}{c}\text { Sun } \\
\text { HPC } 10000400 \mathrm{MHz} \text { Cluster }\end{array}$ & $\begin{array}{c}\text { E-commerce } \\
\text { London UK /2000 }\end{array}$ & $\begin{array}{l}\text { Industry } \\
\text { WWW }\end{array}$ & 96 & $\begin{array}{r}\mathbf{5 9 . 0 4} \\
76.8\end{array}$ & \\
\hline 468 & $\begin{array}{c}\text { Hitachi } \\
\text { SR2201/256 }\end{array}$ & $\begin{array}{c}\text { Hitachi Mechanical Engineering Res. Lab. } \\
\text { Japan /1998 }\end{array}$ & Research & 256 & $\begin{array}{r}\mathbf{5 8 . 6 8} \\
77\end{array}$ & $\begin{array}{l}77760 \\
13440\end{array}$ \\
\hline 469 & $\begin{array}{c}\text { Hitachi } \\
\text { SR2201/256 }\end{array}$ & $\begin{array}{l}\text { Real World Computing (RWCP) } \\
\text { Tokyo Japan /1997 }\end{array}$ & Research & 256 & $\begin{array}{r}58.68 \\
77\end{array}$ & $\begin{array}{l}77760 \\
13440\end{array}$ \\
\hline 470 & $\begin{array}{c}\text { Hitachi } \\
\text { SR2201/256 }\end{array}$ & $\begin{array}{l}\text { University of Cambridge } \\
\text { Cambridge UK /1998 }\end{array}$ & Academic & 256 & $\begin{array}{r}58.68 \\
77\end{array}$ & $\begin{array}{l}77760 \\
13440\end{array}$ \\
\hline 471 & $\begin{array}{c}\text { Hitachi } \\
\text { SR2201/256 }\end{array}$ & $\begin{array}{c}\text { University of Tokyo/Human Genome Center, IMS } \\
\text { Tokyo Japan /1998 }\end{array}$ & Academic & 256 & $\begin{array}{r}\mathbf{5 8 . 6 8} \\
77\end{array}$ & $\begin{array}{l}77760 \\
13440\end{array}$ \\
\hline 472 & $\begin{array}{c}\text { SGI } \\
\text { ORIGIN } 2000\end{array}$ & $\begin{array}{l}\text { Boston University } \\
\text { Boston USA / } 1997\end{array}$ & Academic & 192 & $\begin{array}{l}\mathbf{5 8 . 6} \\
74.88\end{array}$ & \\
\hline 473 & $\begin{array}{c}\text { IBM } \\
\text { SP PC604e } 332 \mathrm{MHz}\end{array}$ & $\begin{array}{l}\text { Lloyds Bank Plc } \\
\text { UK / } 2000\end{array}$ & $\begin{array}{l}\text { Industry } \\
\text { Finance }\end{array}$ & 138 & $\begin{array}{r}\mathbf{5 8 . 4} \\
91.6\end{array}$ & \\
\hline 474 & $\begin{array}{c}\text { IBM } \\
\text { SP Power3 } 200 \mathrm{MHz}\end{array}$ & $\begin{array}{c}\text { Geco-Prakla } \\
\text { Gatwick UK /2000 }\end{array}$ & $\begin{array}{l}\text { Industry } \\
\text { Geophysics }\end{array}$ & 96 & $\begin{array}{r}\mathbf{5 8 . 3} \\
76.6\end{array}$ & \\
\hline 475 & $\begin{array}{c}\text { IBM } \\
\text { SP Power3 } 200 \mathrm{MHz}\end{array}$ & $\begin{array}{c}\text { Volvo } \\
\text { Gothenberg Sweden /2000 }\end{array}$ & $\begin{array}{l}\text { Industry } \\
\text { Automotive }\end{array}$ & 96 & $\begin{array}{r}58.3 \\
76.6\end{array}$ & \\
\hline 476 & $\begin{array}{l}\text { Cray Inc. } \\
\text { T3E }\end{array}$ & $\begin{array}{l}\text { AWI (Alfred Wegener Institut) } \\
\text { Bremerhaven Germany /1998 }\end{array}$ & Research & 134 & $\begin{array}{r}58.2 \\
80.4\end{array}$ & \\
\hline 477 & $\begin{array}{l}\text { Cray Inc. } \\
\text { T3E }\end{array}$ & $\begin{array}{c}\text { Japan Adv. Inst. of Science and Technology (JAIST) } \\
\text { Hokuriku Japan /1997 }\end{array}$ & Academic & 134 & $\begin{array}{r}58.2 \\
80.4\end{array}$ & \\
\hline 478 & $\begin{array}{l}\text { Cray Inc. } \\
\text { T3E }\end{array}$ & $\begin{array}{c}\text { Technical University Delft (TUD) } \\
\text { Delft Netherlands /1997 }\end{array}$ & Academic & 134 & $\begin{array}{r}\mathbf{5 8 . 2} \\
80.4\end{array}$ & \\
\hline 479 & $\begin{array}{c}\text { Fujitsu } \\
\text { VPP700/26E }\end{array}$ & $\begin{array}{c}\text { Meteo-France } \\
\text { Toulouse France /1997 }\end{array}$ & $\begin{array}{l}\text { Research } \\
\text { Weather }\end{array}$ & 26 & $\begin{array}{r}58 \\
62.4\end{array}$ & $\begin{array}{r}74880 \\
5200\end{array}$ \\
\hline 480 & $\begin{array}{c}\text { IBM } \\
\text { SP PC604e } 332 \mathrm{MHz}\end{array}$ & $\begin{array}{c}\text { AI Informatics GmbH (AII) } \\
\text { Austria } / 2000\end{array}$ & $\begin{array}{l}\text { Industry } \\
\text { Database }\end{array}$ & 136 & $\begin{array}{r}\mathbf{5 7 . 6} \\
90.2\end{array}$ & \\
\hline
\end{tabular}


TOP500 Supercomputers - Worldwide

\begin{tabular}{|c|c|c|c|c|c|c|}
\hline $\begin{array}{r}\mathbf{N} \\
\text { world }\end{array}$ & $\begin{array}{l}\text { Manufacturer } \\
\text { Computer }\end{array}$ & $\begin{array}{l}\text { Installation Site } \\
\text { Location/Year }\end{array}$ & $\begin{array}{c}\text { Field of } \\
\text { Application }\end{array}$ & $\begin{array}{c}\# \\
\text { Proc. }\end{array}$ & $\begin{array}{r}\mathbf{R}_{\text {max }} \\
R_{\text {peak }} \\
\text { [Gflop/s] }\end{array}$ & $\begin{array}{r}N_{\max } \\
N_{1 / 2}\end{array}$ \\
\hline 481 & $\begin{array}{c}\text { IBM } \\
\text { SP PC604e } 332 \mathrm{MHz}\end{array}$ & $\begin{array}{l}\text { Communications Company } \\
\text { Japan } / 2000\end{array}$ & Industry & 136 & $\begin{array}{r}\mathbf{5 7 . 6} \\
90.2\end{array}$ & \\
\hline 482 & $\begin{array}{c}\text { IBM } \\
\text { SP PC604e } 332 \mathrm{MHz}\end{array}$ & $\begin{array}{c}\text { France Telecom Mobile Services (FTMS) } \\
\text { France } / 2000\end{array}$ & Industry & 136 & $\begin{array}{r}\mathbf{5 7 . 6} \\
90.2\end{array}$ & \\
\hline 483 & $\begin{array}{c}\text { IBM } \\
\text { SP PC604e } 332 \mathrm{MHz}\end{array}$ & $\begin{array}{c}\text { Oracle Corporation } \\
\text { Redwood Shores USA /1999 }\end{array}$ & $\begin{array}{l}\text { Industry } \\
\text { Database }\end{array}$ & 136 & $\begin{array}{r}\mathbf{5 7 . 6} \\
90.2\end{array}$ & \\
\hline 484 & $\begin{array}{c}\text { IBM } \\
\text { SP PC604e } 332 \mathrm{MHz}\end{array}$ & $\begin{array}{l}\text { Procter and Gamble } \\
\text { Belgium /1999 }\end{array}$ & Industry & 136 & $\begin{array}{r}\mathbf{5 7 . 6} \\
90.2\end{array}$ & \\
\hline 485 & $\begin{array}{l}\text { Cray Inc. } \\
\text { T3E }\end{array}$ & $\begin{array}{l}\text { Ohio Supercomputer Center } \\
\text { Columbus USA /1997 }\end{array}$ & Academic & 132 & $\begin{array}{r}\mathbf{5 7 . 4} \\
79.2\end{array}$ & \\
\hline 486 & $\begin{array}{c}\text { IBM } \\
\text { SP P2SC } 160 \mathrm{MHz}\end{array}$ & $\begin{array}{c}\text { Oracle/IBM } \\
\text { France } / 1998\end{array}$ & $\begin{array}{l}\text { Industry } \\
\text { Database }\end{array}$ & 128 & $\begin{array}{r}57.24 \\
81.92\end{array}$ & $\begin{array}{r}39000 \\
9180\end{array}$ \\
\hline 487 & $\begin{array}{c}\text { Fujitsu } \\
\text { VPP500/40 }\end{array}$ & $\begin{array}{c}\text { National Institute of Genetics } \\
\text { Mishima Japan /1995 }\end{array}$ & Research & 40 & $\begin{array}{r}56.9 \\
64\end{array}$ & \\
\hline 488 & $\begin{array}{c}\text { IBM } \\
\text { SP PC604e } 332 \mathrm{MHz}\end{array}$ & $\begin{array}{l}\text { PO Container Ltd. } \\
\text { UK } / 2000\end{array}$ & $\begin{array}{l}\text { Industry } \\
\text { Database }\end{array}$ & 134 & $\begin{array}{r}\mathbf{5 6 . 7} \\
88.9\end{array}$ & \\
\hline 489 & $\begin{array}{c}\text { IBM } \\
\text { SP PC604e } 332 \mathrm{MHz}\end{array}$ & $\begin{array}{c}\text { Pennsylvania State University } \\
\text { USA / } 1998\end{array}$ & Academic & 134 & $\begin{array}{r}\mathbf{5 6 . 7} \\
88.9\end{array}$ & \\
\hline 490 & $\begin{array}{c}\text { IBM } \\
\text { SP P2SC } 160 \mathrm{MHz}\end{array}$ & $\begin{array}{c}\text { ERDC MSRC } \\
\text { Vicksburg USA /1998 }\end{array}$ & $\begin{array}{l}\text { Industry } \\
\text { Defense }\end{array}$ & 126 & $\begin{array}{r}56.37 \\
80.64\end{array}$ & \\
\hline 491 & $\begin{array}{l}\text { Cray Inc. } \\
\text { T3E1200 }\end{array}$ & $\begin{array}{c}\text { Environmental Protection Agency } \\
\text { USA / } 1999\end{array}$ & Research & 68 & $\begin{array}{r}\mathbf{5 6 . 3} \\
81.6\end{array}$ & \\
\hline 492 & $\begin{array}{c}\text { IBM } \\
\text { SP Power3 } 200 \mathrm{MHz}\end{array}$ & $\begin{array}{l}\text { Government } \\
\text { UK /1998 }\end{array}$ & Classified & 92 & $\begin{array}{r}56 \\
73.4\end{array}$ & \\
\hline 493 & $\begin{array}{c}\text { NEC } \\
\text { SX-5S/16H4 }\end{array}$ & $\begin{array}{c}\text { VW (Volkswagen AG) } \\
\text { Wolfsburg Germany /2000 }\end{array}$ & $\begin{array}{l}\text { Industry } \\
\text { Automotive }\end{array}$ & 16 & $\begin{array}{r}56 \\
64\end{array}$ & \\
\hline 494 & $\begin{array}{c}\text { IBM } \\
\text { SP PC604e } 332 \mathrm{MHz}\end{array}$ & $\begin{array}{l}\text { Auchan } \\
\text { France /1999 }\end{array}$ & $\begin{array}{l}\text { Industry } \\
\text { Database }\end{array}$ & 132 & $\begin{array}{r}\mathbf{5 5 . 9} \\
87.6\end{array}$ & \\
\hline 495 & $\begin{array}{c}\text { IBM } \\
\text { SP S80s } 450 \mathrm{MHz}\end{array}$ & $\begin{array}{c}\text { BASF } \\
\text { Ludwigshafen Germany } / 2000\end{array}$ & $\begin{array}{l}\text { Industry } \\
\text { Chemistry }\end{array}$ & 108 & $\begin{array}{r}\mathbf{5 5 . 8} \\
97.2\end{array}$ & \\
\hline 496 & $\begin{array}{c}\text { IBM } \\
\text { SP P2SC } 160 \mathrm{MHz}\end{array}$ & $\begin{array}{c}\text { Government } \\
\text { France /1999 }\end{array}$ & Classified & 124 & $\begin{array}{r}\mathbf{5 5 . 5} \\
79.3\end{array}$ & \\
\hline 497 & $\begin{array}{c}\text { IBM } \\
\text { SP Power3 } 375 \mathrm{MHz}\end{array}$ & $\begin{array}{c}\text { AP } \\
\text { USA } / 2000\end{array}$ & Industry & 52 & $\begin{array}{r}55.3 \\
78\end{array}$ & \\
\hline 498 & $\begin{array}{c}\text { IBM } \\
\text { SP Power3 } 375 \mathrm{MHz}\end{array}$ & $\begin{array}{c}\text { New York City - Department of Finance } \\
\text { USA } / 2000\end{array}$ & Government & 52 & $\begin{array}{r}55.3 \\
78\end{array}$ & \\
\hline 499 & $\begin{array}{c}\text { IBM } \\
\text { SP Power3 } 375 \mathrm{MHz}\end{array}$ & $\begin{array}{l}\text { Zurich American } \\
\text { USA / } 2000\end{array}$ & Industry & 52 & $\begin{array}{r}\mathbf{5 5 . 3} \\
78\end{array}$ & \\
\hline 500 & $\begin{array}{c}\text { IBM } \\
\text { SP PC604e } 332 \mathrm{MHz}\end{array}$ & $\begin{array}{c}\text { Alcatel } \\
\text { France /1999 }\end{array}$ & $\begin{array}{l}\text { Industry } \\
\text { Telecomm }\end{array}$ & 130 & $\begin{array}{r}\mathbf{5 5 . 1} \\
86.3\end{array}$ & \\
\hline
\end{tabular}




\section{Statistics on Manufacturers and Continents}

As basic statistics of the complete list, we give the number of systems installed with respect to the different manufacturers in the different countries or continents (Table 2) as well as the accumulated $R_{\max }$ values (Table 3 ) and $R_{\text {peak }}$ values (Table 4 ) for those systems. More extensive analyses of the situation and its evolution over time can be found in the series of ToP500Reports (Top500Report 1993 [3], 1994 [4], 1995 [5] and, 1996 [6]). Customized statistics can be obtained by using WWW at http://www.top500.org.

Table 2: Number of Systems Installed

\begin{tabular}{|l||r|r|r|r||r|}
\hline \multicolumn{5}{|c|}{ TOP500 Statistics - Number of Systems Installed } \\
\hline & USA/Canada & Europe & Japan & others & Total \\
\hline \hline IBM & 108 & 90 & 12 & 5 & 215 \\
\hline Sun & 47 & 29 & 8 & 8 & 92 \\
\hline SGI & 42 & 17 & 7 & 1 & 67 \\
\hline Cray Inc. & 26 & 17 & 2 & 2 & 47 \\
\hline NEC & 2 & 9 & 10 & 2 & 23 \\
\hline Fujitsu & & 7 & 9 & 1 & 17 \\
\hline Hitachi & & 3 & 13 & & 16 \\
\hline Compaq & 8 & 2 & 1 & & 11 \\
\hline others & 8 & 3 & 1 & & 12 \\
\hline \hline Total & 241 & 177 & 63 & 19 & 500 \\
\hline
\end{tabular}

Mannheim/Tennessee November 2, 2000

Table 3: Installed $\mathbf{R}_{\max }$

\begin{tabular}{|l||r|r|r|r||r|}
\hline \multicolumn{5}{|c|}{ TOP500 Statistics - Installed $R_{\text {max }}[$ Gflop/s] } \\
\hline & USA/Canada & Europe & Japan & others & Total \\
\hline \hline IBM & 26261 & 8940.0 & 1043.3 & 1128.0 & 37372 \\
\hline Sun & 5400.9 & 2730.3 & 683.4 & 718.0 & 9532.6 \\
\hline SGI & 6032.5 & 1380.7 & 647.4 & 71.7 & 8132.3 \\
\hline Cray Inc. & 8266.7 & 5205.5 & 132.7 & 191.4 & 13796 \\
\hline NEC & 365.0 & 896.7 & 1354.1 & 424.0 & 3039.8 \\
\hline Fujitsu & & 1785.5 & 2321.6 & 139.0 & 4246.1 \\
\hline Hitachi & & 1208.7 & 4911.9 & & 6120.6 \\
\hline Compaq & 1821.3 & 310.2 & 61.3 & & 2192.8 \\
\hline others & 3255.8 & 303.3 & 64.7 & & 3623.8 \\
\hline \hline Total & 51403 & 22761 & 11220 & 2672.1 & 88056 \\
\hline
\end{tabular}

Mannheim/Tennessee November 2, 2000 
Table 4: Installed $\mathbf{R}_{\text {peak }}$

\begin{tabular}{|l||r|r|r|r||r|}
\hline \multicolumn{5}{|c|}{ TOP500 Statistics - Installed $R_{\text {peak }}[$ Gflop/s] } \\
\hline & USA/Canada & Europe & \multicolumn{1}{|c|}{ Japan } & others & Total \\
\hline \hline IBM & 45089 & 13921 & 1506.1 & 1635.0 & 62152 \\
\hline Sun & 7689.6 & 3680.0 & 853.2 & 955.2 & 13178 \\
\hline SGI & 9077.6 & 1726.4 & 921.6 & 89.6 & 11815 \\
\hline Cray Inc. & 11998 & 7444.0 & 183.6 & 277.2 & 19903 \\
\hline NEC & 384.0 & 960.0 & 1416.0 & 448.0 & 3208.0 \\
\hline Fujitsu & & 1948.8 & 2556.2 & 144.0 & 4649.0 \\
\hline Hitachi & & 1549.0 & 6080.0 & & 7629.0 \\
\hline Compaq & 2493.2 & 466.5 & 140.0 & & 3099.7 \\
\hline others & 5340.3 & 718.0 & 105.0 & & 6163.2 \\
\hline \hline Total & 82072 & 32414 & 13762 & 3549.0 & 131797 \\
\hline
\end{tabular}

Mannheim/Tennessee November 2, 2000

\section{References}

[1] H. W. Meuer, The Mannheim Supercomputer Statistics 1986-1992 in [3]

[2] J. J. Dongarra, Performance of Various Computers Using Standard Linear Equations Software, Computer Science Department, University of Tennessee, CS-89-85, 1994

[3] J. J. Dongarra, H. W. Meuer and E. Strohmaier, eds. TOP500 Report 1993, University of Mannheim, 1994

[4] J. J. Dongarra, H. W. Meuer and E. Strohmaier, eds. TOP500 Report 1994, SUPERCOMPUTER 60/61, volumne 11, number $2 / 3$, June 1995

[5] J. J. Dongarra, H. W. Meuer and E. Strohmaier, eds.

TOP500 Report 1995, SUPERCOMPUTER, volumne 12, number 1, January 1996

[6] J. J. Dongarra, H. W. Meuer and E. Strohmaier, eds.

TOP500 Report 1996, SUPERCOMPUTER, volumne 13, number 1, January 1997 